\title{
Nucleon Form Factors in QCD
}

\author{
V.M. Braun, A. Lenz and M. Wittmann \\ Institut für Theoretische Physik, Universität Regensburg, D-93040 Regensburg, Germany
}

(Dated: August 24, 2018)

\begin{abstract}
We calculate the electromagnetic and the axial form factors of the nucleon within the framework of light cone sum rules (LCSR) to leading order in QCD and including higher twist corrections. In particular we motivate a certain choice for the interpolating nucleon field. We find that a simple model of the nucleon distribution amplitudes which deviate from their asymptotic shape, but much less compared to the QCD sum rule estimates, allows one to describe the data remarkably well.
\end{abstract}

PACS numbers: 12.38.-t, 14.20.Dh; 13.40.Gp

\section{INTRODUCTION}

Form factors play an extremely important role in the studies of the internal structure of composite particles as the measure of charge and current distributions. In particular, the pioneering study of the nucleon form factors by Hofstadter and collaborators 1 demonstrated that the nucleons have a finite size of the order of a fermi. The behavior of the form factors at large momentum transfers is especially interesting. Already in the pre-QCD times it was established that, if one can treat the hadrons at high momentum transfer as collinear beams of $N$ valence quarks located at small transverse separations and exchanging intermediate gluing particles with which they interact via a dimensionless coupling constant, then the spin-averaged form factor behaves asymptotically as $1 /\left(Q^{2}\right)^{N-1}$ 2]. This hard-exchange picture and the resulting dimensional power counting rules 2, 3] can be formally extended onto other hard exclusive processes.

After the advent of quantum chromodynamics, this hard-gluon-exchange picture was formalized with the help of the QCD factorization approach to exclusive processes $[4,[5,6]$. This approach introduces the concept of hadron distribution amplitudes (DAs). They are fundamental nonperturbative functions describing the momentum distributions within rare parton configurations when the hadron is represented by a fixed number of Fock constituents (quarks, antiquarks and gluons). It was shown that in the $Q^{2} \rightarrow \infty$ limit, form factors can be written in a factorized form, as a convolution of distribution amplitudes related to hadrons in the initial and final state times a "shortdistance" coefficient function that is calculable in QCD perturbation theory. The leading contribution corresponds to DAs with minimal possible number of constituents - three for baryons and two for mesons.

The essential requirement for the applicability of this approach is a high virtuality of the exchanged gluons and also of the quarks inside the short distance subprocess. More generally, in the case of the nucleon form factors the hard perturbative QCD (pQCD) contribution is only the third term of the factorization expansion. Schematically, one can envisage the expansion of, say, the Dirac electromagnetic nucleon form factor $F_{1}\left(Q^{2}\right)$ of the form

$$
\begin{aligned}
F_{1}\left(Q^{2}\right) \sim & A\left(Q^{2}\right)+\left(\frac{\alpha_{s}\left(Q^{2}\right)}{\pi}\right) \frac{B\left(Q^{2}\right)}{Q^{2}} \\
& +\left(\frac{\alpha_{s}\left(Q^{2}\right)}{\pi}\right)^{2} \frac{C}{Q^{4}}+\ldots
\end{aligned}
$$

where $C$ is a constant determined by the nucleon DAs, while $A\left(Q^{2}\right)$ and $B\left(Q^{2}\right)$ are form-factor-type functions generated by contributions of low virtualities, see Fig. 1 The soft functions $A\left(Q^{2}\right)$ and $B\left(Q^{2}\right)$ are purely nonperturbative and cannot be further simplified e.g. factorized in terms of DAs. In the light-cone formalism, they are determined by overlap integrals of the soft parts of hadronic wave functions corresponding to large transverse separations. Various estimates suggest that $A\left(Q^{2}\right) \lesssim 1 / Q^{6}, B\left(Q^{2}\right) \lesssim 1 / Q^{4}$ and at very large $Q^{2}$ they are further suppressed by the $\mathrm{Su}-$ dakov form factor. To be precise, in higher orders in $\alpha_{s}(Q)$ there exist double-logarithmic contributions $\sim 1 / Q^{4}[]$ that are not factorized in the standard manner; however, also they are suppressed by the Sudakov mechanism [8, 9, 10]. Thus, the third term in (11) is formally the leading one at large $Q^{2}$ to power accuracy.

The main problem of the $\mathrm{pQCD}$ approach is a numerical suppression of each hard gluon exchange by the $\alpha_{s} / \pi$ factor which is a standard perturbation theory penalty for each extra loop. If, say, $\alpha_{s} / \pi \sim 0.1$, the pQCD contribution to baryon form factors is suppressed by a factor of $100 \mathrm{com}-$ pared to the purely soft term. As the result, the onset of the perturbative regime is postponed to very large momentum transfers since the factorizable pQCD contribution $O\left(1 / Q^{4}\right)$ has to win over 


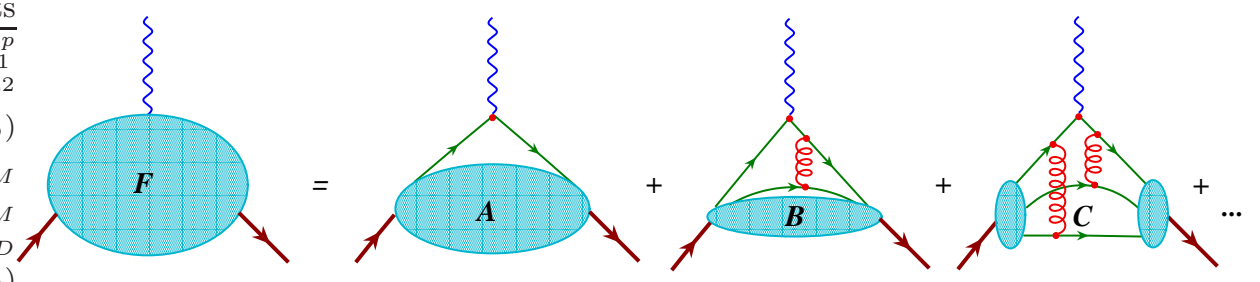

FIG. 1: Structure of QCD factorization for baryon form factors.

nonperturbative effects that are suppressed by extra powers of $1 / Q^{2}$, but do not involve small coefficients. There is a growing consensus that "soft" contributions play the dominant role at present energies. Indeed, it is known for a long time that the use of QCD-motivated models for the wave functions allows one to obtain, without much effort, soft contributions comparable in size to experimentally observed values (see, e.g. 11, 12, 13]). A modern trend 14, 15] is to use the concept of generalized parton distributions (GPDs) to describe/parametrize soft contributions in various exclusive reactions, see 16, 17, 18 for recent reviews, and the models of GPDs usually are chosen such that the experimental data on form factors are described by the soft contributions alone, cf. Refs. 19, 20, 21]. A subtle point for these semiphenomenological approaches is to avoid double counting of hard rescattering contributions "hidden" in the model-dependent hadron wave functions or GPD parametrizations.

The dominant role of the soft contribution for the pion form factor at moderate momentum transfers, up to $Q^{2} \sim 2-3 \mathrm{GeV}^{2}$, is supported by its calculation 22, 23 within the QCD sum rule approach [24]. The application of the method at higher $Q^{2}$ faces the problem that the inclusion of nonperturbative effects due to vacuum condensates through the expansion over inverse powers of the Borel parameter $M^{2}$ interferes with the large- $Q^{2}$ expansion of the form factors, producing an illbehaved series of the type $\sum_{n} c_{n}\left(Q^{2} / M^{2}\right)^{n}$. For the nucleon form factors, the QCD sum rule approach only works in the region of small momentum transfers $Q^{2}<1 \mathrm{GeV}^{2}$ [25, 26]. To extend the results to higher $Q^{2}$, it was proposed 27] to resum the $\left(Q^{2} / M^{2}\right)^{n}$ contributions originating from the Taylor expansion of simple models for nonlocal condensates. Another approach [23, 28] is to use the so-called local quark-hadron duality approximation, in which the condensates are effectively neglected. The parameter-free results for the pion and nucleon form factors obtained in this way are in a rather good agreement with the existing data.
We also have to mention the dispersion approach of Ref. [29, 30] which allows to analyze form factors for all momenta (space- and time-like) in a largely model-independent manner in terms of spectral functions on a hadronic level. Also, in future, one expects that the rapid development of lattice QCD will allow one to calculate baryon form factors to sufficient precision from first principles, see e.g. 31, 32, 33, 34]. Such studies are necessary and interesting in its own right, but do not add to our understanding of how QCD actually "works" to transfer the large momentum along the nucleon constituents, the quarks and gluons. The main motivation to study "hard" processes has always been to understand hadron properties in terms of quark and gluon degrees of freedom; for example, the rationale for the continuing measurements of the total inclusive cross section in deep inelastic scattering is to extract quark and gluon parton distributions. Similar, experimental measurements of the form factors at large momentum transfers should eventually allow one to determine baryon distribution amplitudes and this task is obscured by the presence of large "soft" contributions which have to be subtracted.

In Ref. 35 we have suggested to calculate baryon form factors for moderately large $Q^{2}$ using light-cone sum rules (LCSR) 36, 37. This technique is attractive because in LCSRs "soft" contributions to the form factors are calculated in terms of the same DAs that enter the pQCD calculation and there is no double counting. Thus, the LCSRs provide one with the most direct relation of the hadron form factors and distribution amplitudes that is available at present, with no other nonperturbative parameters.

The basic object of the LCSR approach is the correlation function

$$
\int d x e^{-i q x}\langle 0|T\{\eta(0) j(x)\}| N(P)\rangle
$$

in which $j$ represents the electromagnetic (or weak) probe and $\eta$ is a suitable operator with nucleon quantum numbers. The other (in this example, initial state) nucleon is explicitly represented by its 
state vector $|N(P)\rangle$, see a schematic representation in Fig. 2] When both the momentum transfer $Q^{2}$

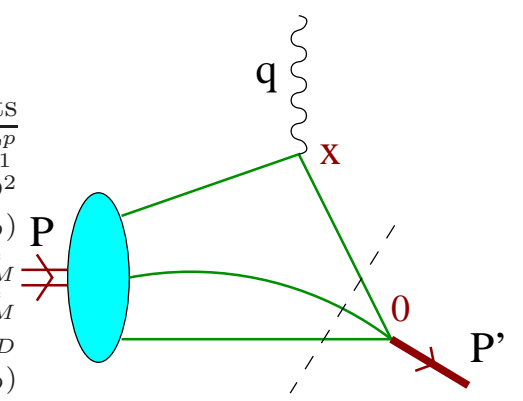

FIG. 2: Schematic structure of the light-cone sum rule for baryon form factors.

and the momentum $\left(P^{\prime}\right)^{2}=(P-q)^{2}$ flowing in the $\eta$ vertex are large and negative, the asymptotics of the correlation function is governed by the light-cone kinematics $x^{2} \rightarrow 0$ and can be studied using the operator product expansion (OPE) $T\{\eta(0) j(x)\} \sim \sum C_{i}(x) \mathcal{O}_{i}(0)$ on the light-cone $x^{2}=0$. The $x^{2}$-singularity of a particular perturbatively calculable short-distance factor $C_{i}(x)$ is determined by the twist of the relevant composite operator $\mathcal{O}_{i}$, whose matrix element $\left\langle 0\left|\mathcal{O}_{i}(0)\right| N(P)\right\rangle$ is given by an appropriate moment of the nucleon DA. Next, one can represent the answer in form of the dispersion integral in $\left(P^{\prime}\right)^{2}$ and define the nucleon contribution by the cutoff in the quarkantiquark invariant mass, the so-called interval of duality $s_{0}$ (or continuum threshold). The main role of the interval of duality is that it does not allow large momenta $\left|k^{2}\right|>s_{0}$ to flow through the $\eta$ vertex; to the lowest order $O\left(\alpha_{s}^{0}\right)$ one obtains a purely soft contribution to the form factor as a sum of terms ordered by twist of the relevant operators and hence including both the leading- and the higher-twist nucleon DAs. Note that, in difference to the hard mechanism, the contribution of higher-twist DAs is only suppressed by powers of $\left|\left(P^{\prime}\right)^{2}\right| \sim 1-2 \mathrm{GeV}^{2}$ (which is translated to the suppression by powers of the Borel parameter after applying the usual QCD sum rule machinery), but not by powers of $Q^{2}$. This feature is in agreement with the common wisdom that soft contributions are not constrained to small transverse separations.

The LCSR expansion also contains terms generating the asymptotic pQCD contributions. They appear at proper order in $\alpha_{s}$, i.e., in the $O\left(\alpha_{s}\right)$ term for the pion form factor, at the $\alpha_{s}^{2}$ order for the nucleon form factors, etc. In the pion case, it was explicitly demonstrated [38, 39] that the contribution of hard rescattering is correctly reproduced in the LCSR approach as a part of the $O\left(\alpha_{s}\right)$ correction. It should be noted that the diagrams of LCSR that contain the "hard" pQCD contributions also possess "soft" parts, i.e., one should perform a separation of "hard" and "soft" terms inside each diagram. As a result, the distinction between "hard" and "soft" contributions appears to be scale- and scheme-dependent [38]. During the last years there have been numerous applications of LCSRs to mesons, see [40, 41] for a review. Following the work 35] nucleon form factors were further considered in this framework in Refs. [42, 43, 44] and the weak decay $\Lambda_{b} \rightarrow p \ell \nu_{\ell}$ in [45]. The generalization to the $N \gamma \Delta$ transition form factor was worked out in [46].

In this paper, we go beyond the original work 35] in several important aspects. First, we present a detailed study using different interpolating currents for the nucleon and choose one which appears to be the optimal. Second, we calculate both the electromagnetic and weak decay form factors. Third, we make an update of the parameters of higher-twist DAs which feature prominently in this approach and are important numerically. We then formulate a simple model for the DAs that provides a good description of the available experimental data. Finally, we include a complete summary of higher-twist DAs and work out the light-cone expansion of three-quark currents for all Lorentz structures, which extends the results given in [35]. The presentation is organized as follows. Section 2 is introductory and contains the form factor definitions and some general discussion. Section 3 is devoted to the construction of the sum rules. We present here our numerical results and the conclusions. The final section 4 is reserved for a short summary and an outlook. The paper contains five appendices devoted to a summary of correlation functions to tree-level accuracy, summary of threequark nucleon distribution amplitudes, the OPE of generic three-quark amplitudes to twist-5 accuracy, and the QCD sum rule estimates for the parameters of higher-twist DAs.

\section{PRELIMINARIES}

\section{A. Electromagnetic Form Factors of the Nucleon}

The matrix element of the electromagnetic current

$$
j_{\mu}^{\mathrm{em}}(x)=e_{u} \bar{u}(x) \gamma_{\mu} u(x)+e_{d} \bar{d}(x) \gamma_{\mu} d(x)
$$

taken between nucleon states is conventionally written in terms of the Dirac and Pauli form factors 
$F_{1}\left(Q^{2}\right)$ and $F_{2}\left(Q^{2}\right)$ :

$$
\begin{aligned}
& \left\langle N\left(P^{\prime}\right)\left|j_{\mu}^{\mathrm{em}}(0)\right| N(P)\right\rangle= \\
& =\bar{N}\left(P^{\prime}\right)\left[\gamma_{\mu} F_{1}\left(Q^{2}\right)-i \frac{\sigma_{\mu \nu} q^{\nu}}{2 m_{N}} F_{2}\left(Q^{2}\right)\right] N(P),(3)
\end{aligned}
$$

where $P_{\mu}$ is the initial nucleon momentum, $P^{2}=$ $m_{N}^{2}, P^{\prime}=P-q, Q^{2}:=-q^{2}, \sigma_{\mu \nu}=\frac{i}{2}\left[\gamma_{\mu}, \gamma_{\nu}\right]$ and $N(P)$ is the nucleon spinor.

Experimental data on the scattering of electrons off nucleons, e.g. $e^{-}+p \rightarrow e^{-}+p$, is usually presented in terms of the electric $G_{E}\left(Q^{2}\right)$ and magnetic $G_{M}\left(Q^{2}\right)$ Sachs form factors which are related to $F_{1,2}\left(Q^{2}\right)$ as

$$
\begin{aligned}
& G_{M}\left(Q^{2}\right)=F_{1}\left(Q^{2}\right)+F_{2}\left(Q^{2}\right), \\
& G_{E}\left(Q^{2}\right)=F_{1}\left(Q^{2}\right)-\frac{Q^{2}}{4 m_{N}^{2}} F_{2}\left(Q^{2}\right) .
\end{aligned}
$$

In the Breit frame the form factors $G_{E}\left(Q^{2}\right)$ and $G_{M}\left(Q^{2}\right)$ can be thought of, loosely speaking, as the Fourier transforms of the charge distribution and magnetization density in the nucleon.

The normalization of the form factors at $Q^{2}=0$ is given by the nucleon charges and magnetic moments

$$
\begin{aligned}
& G_{E}^{p}(0)=1, G_{M}^{p}(0)=\mu_{p}=2.792847337(29), \\
& G_{E}^{n}(0)=0, G_{M}^{n}(0)=\mu_{n}=-1.91304272(45)(6)
\end{aligned}
$$

for the proton and the neutron, respectively 47].

Experimentally one finds 48, 49, 50, 51, 52, 53, $54,55,56,57,58$ that the magnetic form factors of both the proton and the neutron can be described by the famous dipole formula:

$$
\begin{aligned}
& G_{M}^{p}\left(Q^{2}\right)=\frac{\mu_{p}}{\left(1+\frac{Q^{2}}{\mu_{0}^{2}}\right)^{2}}, \\
& G_{M}^{n}\left(Q^{2}\right)=\frac{\mu_{n}}{\left(1+\frac{Q^{2}}{\mu_{0}^{2}}\right)^{2}},
\end{aligned}
$$

with $\mu_{0}^{2}=0.71 \mathrm{GeV}^{2}$. For the electric form factor of the neutron the measured values are close to zero [59, 60]. In the case of the electric form factor of the proton the situation was controversial for some time, with the experimental measurements using the classical method of Rosenbluth separation producing very different results compared to the ones obtained using the method of polarization transfer. Recently it was argued 61, 62, 63, 64, 65, 66, 67, 68, 69] that the former approach is not applicable for sufficiently large momentum transfers, as the contribution of the electric form factor to the spin-averaged cross section is strongly contaminated by contributions of the two-photon exchange. The existing estimates 61, 62, 63, 64, 65, 66, 67, 68, 69 indicate that the two-photon exchange corrections have the right sign and order of magnitude to bring the values obtained via the Rosenbluth separation to the ones measured using the polarization transfer, although the situation is not finally settled. In this work we rely on the polarization transfer data $70,71,72]$.

\section{B. Charged Weak Form Factors}

In order to describe charged current (CC) neutrino reactions like

$$
\begin{aligned}
& \nu_{\mu}+n \rightarrow \mu^{-}+p, \\
& \bar{\nu}_{\mu}+p \rightarrow \mu^{+}+n,
\end{aligned}
$$

one has to deal with matrix elements between nucleon states of the vector $V_{\mu}^{C C}$ and the axial-vector current $A_{\mu}^{C C}$ :

$$
\begin{aligned}
& V_{\mu}^{C C}(x)=\bar{\Psi} \gamma^{\mu} \tau^{+} \Psi(x), \\
& A_{\mu}^{C C}(x)=\bar{\Psi} \gamma^{\mu} \gamma_{5} \tau^{+} \Psi(x),
\end{aligned}
$$

where $\Psi$ is an (iso)spinor consisting of an up- and a down-quark and $\tau^{+}=1 / 2\left(\tau_{1}+i \tau_{2}\right)$ is a linear combination of the familiar Pauli matrices.

One defines the corresponding vector and the axial-vector form factors as

$$
\begin{aligned}
& \left\langle N\left(P^{\prime}\right)\left|V_{\mu}^{C C}(0)\right| N(P)\right\rangle= \\
& =\bar{N}\left(P^{\prime}\right)\left[\gamma_{\mu} F_{1}^{C C}\left(Q^{2}\right)-i \frac{\sigma_{\mu \nu} q^{\nu}}{2 m_{N}} F_{2}^{C C}\left(Q^{2}\right)\right] N(P), \\
& \left\langle N\left(P^{\prime}\right)\left|A_{\mu}^{C C}(0)\right| N(P)\right\rangle=\bar{N}\left(P^{\prime}\right)\left[\gamma_{\mu} G_{A}^{C C}\left(Q^{2}\right)\right. \\
& \left.\quad-\frac{q_{\mu}}{2 m_{N}} G_{P}^{C C}\left(Q^{2}\right)-i \frac{\sigma_{\mu \nu} q^{\nu}}{2 m_{N}} G_{T}^{C C}\left(Q^{2}\right)\right] \gamma_{5} N(P) .
\end{aligned}
$$

The vector form factors can be related with the electromagnetic ones with the help of isospin symmetry, to wit

$$
\begin{aligned}
\left\langle p\left|\bar{u} \gamma^{\mu} d\right| n\right\rangle & =\left\langle p\left|j_{e m}^{\mu}\right| p\right\rangle-\left\langle n\left|j_{e m}^{\mu}\right| n\right\rangle, \\
\left\langle p\left|\bar{u} \gamma^{\mu} \gamma_{5} d\right| n\right\rangle & =\left\langle p\left|\left(\bar{u} \gamma^{\mu} \gamma_{5} u-\bar{d} \gamma^{\mu} \gamma_{5} d\right)\right| p\right\rangle .
\end{aligned}
$$

The first relation gives, e.g.

$$
\begin{aligned}
& F_{1}^{C C}\left(Q^{2}\right)=F_{1}^{p}\left(Q^{2}\right)-F_{1}^{n}\left(Q^{2}\right), \\
& F_{2}^{C C}\left(Q^{2}\right)=F_{2}^{p}\left(Q^{2}\right)-F_{2}^{n}\left(Q^{2}\right) .
\end{aligned}
$$


The axial form factor $G_{A}^{C C}\left(Q^{2}\right)$ can be determined either from quasi-elastic neutrino scattering or from pion electroproduction (with the help of current algebra). The neutrino data are available for $Q^{2}$ values up to $3 \mathrm{GeV}^{2}$ 73, 74, 75, 76. They were reanalyzed recently in [77]. The pion electroproduction data exist for $Q^{2}<1 \mathrm{GeV}^{2}$, e.g. [78]. After the inclusion of a finite pion mass correction in the analysis [79], the extracted form factor agrees well with the determinations in neutrino scattering. All the existing experimental data for $G_{A}^{C C}\left(Q^{2}\right)$ at $Q^{2}<1 \mathrm{GeV}^{2}$ are very well described by the dipole formula:

$$
G_{A}^{C C}\left(Q^{2}\right)=\frac{g_{A}}{\left(1+\frac{Q^{2}}{M_{A}^{2}}\right)^{2}},
$$

with $g_{A}=1.267 \pm 0.004$. The mass parameter is fitted to be $M_{A}=1.001 \pm 0.020 \mathrm{GeV}$ and $M_{A}=$ $1.013 \pm 0.015 \mathrm{GeV}$ from neutrino scattering and pion electroproduction, respectively.

The pseudoscalar form factor $G_{P}^{C C}\left(Q^{2}\right)$ can be extracted separately from muon capture of the proton $\mu^{-}+p \rightarrow \nu_{\mu}+n$ or from pion electroproduction. In this case only the data for $Q^{2}$-values below $0.2 \mathrm{GeV}^{2}$ exist [80], which is to low for the application of our method. Using PCAC and the pion pole dominance model one can express the pseudoscalar form factor $G_{P}^{C C}$ in terms of the axial form factor $G_{A}^{C C}$ :

$$
G_{P}^{C C}\left(Q^{2}\right)=\frac{4 m_{N}^{2} G_{A}^{C C}\left(Q^{2}\right)}{Q^{2}+m_{\pi}^{2}} .
$$

This form is consistent with the conservation of the flavor nonsinglet axial current in the chiral limit $\left(m_{\pi}^{2} \rightarrow 0\right)$.

Finally, the tensor form factor $G_{T}^{C C}\left(Q^{2}\right)$ must vanish by virtue of the isospin symmetry and $\mathrm{T}$ invariance, so it is normally not included. The reason why we leave it in Eq. (13) is that in our approach the initial and the final state nucleons are treated differently, so that T-invariance is not manifest.

\section{Neutral Weak Form Factors}

The cross section for elastic neutrino-proton and neutrino-neutron scattering can be expressed in terms of matrix elements of a vector $V_{\mu}^{N C}$ and an axial-vector $A_{\mu}^{N C}$ neutral currents:

$$
V_{\mu}^{N C}(x)=\frac{1}{2}\left[\left(1-\frac{8}{3} \sin ^{2} \theta_{W}\right) \bar{u} \gamma^{\mu} u(x)\right.
$$

$$
\begin{array}{r}
\left.-\left(1-\frac{4}{3} \sin ^{2} \theta_{W}\right) \bar{d} \gamma^{\mu} d(x)\right], \\
A_{\mu}^{N C}(x)=\frac{1}{2}\left[\bar{u} \gamma^{\mu} \gamma_{5} u(x)-\bar{d} \gamma^{\mu} \gamma_{5} d(x)\right],
\end{array}
$$

where $\theta_{W}$ is the Weinberg angle. The matrix elements of neutral currents over the nucleon states are conventionally written as

$$
\begin{aligned}
& \left\langle N\left(P^{\prime}\right)\left|V_{\mu}^{N C}(0)\right| N(P)\right\rangle= \\
& =\bar{N}\left(P^{\prime}\right)\left[\gamma_{\mu} F_{1}^{N C}\left(Q^{2}\right)-i \frac{\sigma_{\mu \nu} q^{\nu}}{2 m_{N}} F_{2}^{N C}\left(Q^{2}\right)\right] N(P) \\
& \left\langle N\left(P^{\prime}\right)\left|A_{\mu}^{N C}(0)\right| N(P)\right\rangle=\bar{N}\left(P^{\prime}\right)\left[\gamma_{\mu} G_{A}^{N C}\left(Q^{2}\right)\right. \\
& \left.\quad-\frac{q_{\mu}}{2 m_{N}} G_{P}^{N C}\left(Q^{2}\right)-i \frac{\sigma_{\mu \nu} q^{\nu}}{2 m_{N}} G_{T}^{N C}\left(Q^{2}\right)\right] \gamma_{5} N(P) .
\end{aligned}
$$

The vector form factors $F_{1}^{N C}$ and $F_{2}^{N C}$ are, again, just linear combinations of the electromagnetic form factors of the nucleon. For the axial neutral weak form factors $\left(G_{A}^{N C}\right.$ and $\left.G_{P}^{N C}\right)$ there is little data, and only for $Q^{2}<1 \mathrm{GeV}^{2}$ 81 which is below the region we try to describe theoretically. The tensor form factor $G_{T}^{N C}\left(Q^{2}\right)$ must vanish by virtue of T-invariance; the reason we include it will become clear later.

\section{Light-Cone Kinematics}

Having in mind the practical construction of light-cone sum rules that involve nucleon DAs, we define a light-like vector $z_{\mu}$ by the condition

$$
q \cdot z=0, \quad z^{2}=0
$$

and introduce the second light-like vector vector

$$
p_{\mu}=P_{\mu}-\frac{1}{2} z_{\mu} \frac{m_{N}^{2}}{P \cdot z}, \quad p^{2}=0,
$$

so that $P \rightarrow p$ if the nucleon mass can be neglected, $m_{N} \rightarrow 0$. The photon momentum can be written as

$$
q_{\mu}=q_{\perp \mu}+z_{\mu} \frac{P \cdot q}{P \cdot z} .
$$

We also need the projector onto the directions orthogonal to $p$ and $z$,

$$
g_{\mu \nu}^{\perp}=g_{\mu \nu}-\frac{1}{p z}\left(p_{\mu} z_{\nu}+p_{\nu} z_{\mu}\right),
$$

and use the notation

$$
a_{z} \equiv a_{\mu} z^{\mu}, \quad a_{p} \equiv a_{\mu} p^{\mu},
$$


for arbitrary Lorentz vectors $a_{\mu}$. In turn, $a_{\perp}$ denotes the generic component of $a_{\mu}$ orthogonal to $z$ and $p$, in particular

$$
q_{\perp \mu}=q_{\mu}-\frac{p q}{p z} z_{\mu} .
$$

We use the standard Bjorken-Drell convention 82 for the metric and the Dirac matrices; in particular, $\gamma_{5}=i \gamma^{0} \gamma^{1} \gamma^{2} \gamma^{3}$, and the Levi-Civita tensor $\epsilon_{\mu \nu \lambda \sigma}$ is defined as the totally antisymmetric tensor with $\epsilon_{0123}=1$.

Assume for a moment that the nucleon moves in the positive $\mathbf{e}_{\mathbf{z}}$ direction, then $p^{+}$and $z^{-}$are the only nonvanishing components of $p$ and $z$, respectively. The infinite momentum frame can be visualized as the limit $p^{+} \sim Q \rightarrow \infty$ with fixed $P \cdot z=p \cdot z \sim 1$ where $Q$ is the large scale in the process. Expanding the matrix element in powers of $1 / p^{+}$introduces the power counting in $Q$. In this language, twist counts the suppression in powers of $p^{+}$. Similarly, the nucleon spinor $N_{\gamma}(P, \lambda)$ has to be decomposed in "large" and "small" components as

$$
\begin{aligned}
N_{\gamma}(P, \lambda) & =\frac{1}{2 p \cdot z}(\not p \not \not+\not \not p) N_{\gamma}(P, \lambda) \\
& =N_{\gamma}^{+}(P, \lambda)+N_{\gamma}^{-}(P, \lambda),
\end{aligned}
$$

where we have introduced two projection operators

$$
\Lambda^{+}=\frac{\not p \not z}{2 p \cdot z} \quad, \quad \Lambda^{-}=\frac{\not \not p}{2 p \cdot z}
$$

that project onto the "plus" and "minus" components of the spinor. Note the useful relations

$$
\not p N(P)=m_{N} N^{+}(P), \quad \not k N(P)=\frac{2 p \cdot z}{m_{N}} N^{-}(P)
$$

that are a consequence of the Dirac equation $\not P N(P)=m_{N} N(P)$. Using the explicit expressions for $N(P)$ it is easy to see that $\Lambda^{+} N=N^{+} \sim \sqrt{p^{+}}$ while $\Lambda^{-} N=N^{-} \sim 1 / \sqrt{p^{+}}$.

Note that all expressions are invariant under the reparametrization $z_{\mu} \rightarrow \alpha z_{\mu}$ where $\alpha$ is a real number; we will use this freedom to set $z_{\mu}$ equal to the "minus" component of the distance between the currents in the operator product.

\section{LIGHT-CONE SUM RULES FOR BARYON FORM FACTORS}

\section{A. Choice of the current}

As already mentioned, in the LCSR approach one of the participating nucleons is replaced by a suitable interpolating current for which there are several choices. Altogether, there exist three local operators with isospin $I=1 / 2$ numbers that do not involve derivatives 84 . They can be chosen as

$$
\begin{aligned}
\eta_{1}(x)= & \varepsilon^{i j k}\left[u^{i}(x) C \gamma_{\mu} u^{j}(x)\right] \gamma_{5} \gamma^{\mu} d^{k}(x), \\
\eta_{2}(x)= & \varepsilon^{i j k}\left[u^{i}(x) C \sigma_{\mu \nu} u^{j}(x)\right] \gamma_{5} \sigma^{\mu \nu} d^{k}(x),(31) \\
\eta_{3}(x)= & \frac{2}{3} \epsilon^{i j k}\left(\left[u^{i}(x) C \not u^{j}(x)\right] \gamma_{5} \not d^{k}(x)\right. \\
& \left.-\left[u^{i}(x) C \not d^{j}(x)\right] \gamma_{5} \not u^{k}(x)\right)
\end{aligned}
$$

where $u(x)$ and $d(x)$ are the $u$-quark and the $d$ quark field operators, respectively, $i, j, k$ are color indices, $C$ is the charge conjugation matrix [82] and $z$ is a light-like vector, $z^{2}=0$. The corresponding couplings

$$
\begin{aligned}
& \left\langle 0\left|\eta_{1}(0)\right| N(P)\right\rangle=\lambda_{1} m_{N} N(P), \\
& \left\langle 0\left|\eta_{2}(0)\right| N(P)\right\rangle=\lambda_{2} m_{N} N(P), \\
& \left\langle 0\left|\eta_{3}(0)\right| N(P)\right\rangle=f_{\mathrm{N}}(P z) \not N(P)
\end{aligned}
$$

are well known, albeit with limited precision, from the vast $\mathrm{QCD}$ sum rule literature, see Eq. (B.13) in Appendix B for the current estimates.

The operator $\eta_{1}$ is known as the Ioffe-current 85. There is overwhelming evidence (see, however 86]) that this current gives rise to more accurate and reliable sum rules compared to $\eta_{2}$ 87]. By this reason we do not use $\eta_{2}$ in the construction of the LCSRs below. The both constants $\lambda_{1}$ and $\lambda_{2}$ do appear in the sum rules, however, as they determine the normalization of the higher-twist-4 nucleon DAs.

In turn, the operator $\eta_{3}$ is twist-3 and the corresponding coupling $f_{N}$ determines the normalization of the leading twist nucleon DA [88]. Using the currents of lower twist is in general advantageous, as the corresponding correlation functions have lower dimension and are less affected by the model-dependent continuum subtraction. LCSRs obtained with this current are most close in spirit to $\mathrm{pQCD}$ factorization. The price to pay is that this current couples both to the spin $J=1 / 2$ and spin $J=3 / 2$ baryons and it is unclear whether the unwanted $I=3 / 2$ contributions to the correlation function are sufficiently suppressed. This current has been used more rarely in the practice of QCD sum rule calculations, so that there is less experience.

In Ref. 35] a modification of the current (32) was used

$$
\begin{aligned}
& \eta_{4}(x)=\epsilon^{i j k}\left[u^{i}(x) C \not u^{j}(x)\right] \gamma_{5} \not d^{k}(x), \\
& \left\langle 0\left|\eta_{4}(0)\right| N(P)\right\rangle=f_{\mathrm{N}}(P z) \not \not N(P),
\end{aligned}
$$


which, in difference to $\eta_{3}$, also couples to the isospin $I=3 / 2$ states (e.g. the $\Delta$-isobar).

A priory, it is not obvious whether using the pure isospin- $1 / 2$ or, similarly, pure spin- $1 / 2$ currents improves the accuracy of the sum rules. It is conceivable that the summation over quantum numbers makes the duality approximation for taking into account contributions of heavy resonances and the continuum more accurate and also suppresses poorly known contributions of higherdimension(twist) operators. In a different context, there have been various proposals to add together correlation functions of opposite parity, 89., use chirally projected quark fields, e.g. 90], etc.

We believe that there is no general recipe; one has to consider each case separately and the conclusions can vary. For the problem at hand, it was noticed in [4] that using the currents $\eta_{3}$ and $\eta_{4}$ one obtains numerical results for the nucleon form factors that differ significantly from one another. In this work we demonstrate that this difference is due to the contamination of the sum rules 35 by the contributions of isospin $I=3 / 2$ states and, therefore, the use of the pure-isospin current $\eta_{3}$ is strongly preferred compared to $\eta_{4}$. On the other hand, the sum rules obtained using $\eta_{1}$ and $\eta_{3}$ are complementary to a large extent and both of them are useful. Nevertheless, using the Ioffe current $\eta_{1}$ produces the sum rules that are more stable and seem to be superior in all respects, which makes this current to be our final choice.

\section{B. Correlation functions}

We consider the set of correlation functions

$$
\begin{gathered}
T_{\nu}^{i, \mathrm{em}}(P, q)=i \int \mathrm{d}^{4} x e^{i q x}\left\langle 0\left|T\left[\eta_{i}(0) j_{\nu}^{\mathrm{em}}(x)\right]\right| N(P)\right\rangle, \\
T_{\nu}^{i, \mathrm{a}}(P, q)=i \int \mathrm{d}^{4} x e^{i q x}\left\langle 0\left|T\left[\eta_{i}(0) j_{\nu}^{\mathrm{a}}(x)\right]\right| N(P)\right\rangle, \\
T_{\nu}^{i, \mathrm{v}}(P, q)=i \int \mathrm{d}^{4} x e^{i q x}\left\langle 0\left|T\left[\eta_{i}(0) j_{\nu}^{\mathrm{v}}(x)\right]\right| N(P)\right\rangle,
\end{gathered}
$$

where $T$ denotes time-ordering, $|N(P)\rangle$ is the proton state with four-momentum $P_{\mu}, P^{2}=m_{N}^{2}, \eta_{i}$ with $i=1,3,4$ are the nucleon currents defined in
(30). Further, $j_{\nu}^{\mathrm{em}}$ is the electromagnetic current defined (21) whereas $j_{\nu}^{\mathrm{a}}$ and $j_{\nu}^{\mathrm{v}}$ are the isospin-one axial and vector currents, respectively:

$$
\begin{aligned}
j_{\nu}^{\mathrm{a}, \mathrm{nc}}(x) & =\frac{1}{2}\left[\bar{u}(x) \gamma_{\nu} \gamma_{5} u(x)-\bar{d}(x) \gamma_{\nu} \gamma_{5} d(x)\right], \\
j_{\nu}^{\mathrm{a}, \mathrm{cc}}(x) & =\bar{u}(x) \gamma_{\nu} \gamma_{5} d(x), \\
j_{\nu}^{\mathrm{v}, \mathrm{nc}}(x) & =\frac{1}{2}\left[\bar{u}(x) \gamma_{\nu} u(x)-\bar{d}(x) \gamma_{\nu} d(x)\right], \\
j_{\nu}^{\mathrm{v}, \mathrm{cc}}(x) & =\bar{u}(x) \gamma_{\nu} d(x) .
\end{aligned}
$$

The correlation functions in (35) involve several invariant functions that can be separated by the appropriate projections. Lorentz structures that are most useful for writing the LCSRs are usually those containing the maximum power of the large momentum $p^{+} \sim p z$. We define, for the Ioffe current

$$
\begin{gathered}
\Lambda_{+} T_{z}^{1, \mathrm{em}}=(p z)\left\{m_{N} \mathcal{A}_{1}^{\mathrm{em}}+\not q_{\perp} \mathcal{B}_{1}^{\mathrm{em}}\right\} N^{+}(P), \\
\Lambda_{+} T_{z}^{1, \mathrm{a}}=(p z)\left\{m_{N} \mathcal{A}_{1}^{\mathrm{a}}+\not q_{\perp} \mathcal{B}_{1}^{\mathrm{a}}\right\} \gamma_{5} N^{+}(P), \\
\Lambda_{+} T_{z}^{1, \mathrm{v}}=(p z)\left\{m_{N} \mathcal{A}_{1}^{\mathrm{v}}+\not q_{\perp} \mathcal{B}_{1}^{\mathrm{v}}\right\} N^{+}(P),
\end{gathered}
$$

where $\mathcal{A}$ and $\mathcal{B}$ depend on the Lorentz-invariants $Q^{2}=-q^{2}$ and $P^{\prime 2}=(P-q)^{2}$. For the leadingtwist current $\eta_{3}$ we use instead

$$
\begin{aligned}
T_{z}^{3, \mathrm{em}} & =\frac{2(p z)^{3}}{m_{N}^{2}}\left\{m_{N} \mathcal{A}_{3}^{\mathrm{em}}+\not q_{\perp} \mathcal{B}_{3}^{\mathrm{em}}\right\} N^{-}(P), \\
T_{z}^{3, \mathrm{a}} & =\frac{2(p z)^{3}}{m_{N}^{2}}\left\{m_{N} \mathcal{A}_{3}^{\mathrm{a}}+\not q_{\perp} \mathcal{B}_{3}^{\mathrm{a}}\right\} \gamma_{5} N^{-}(P), \\
T_{z}^{3, \mathrm{v}} & =\frac{2(p z)^{3}}{m_{N}^{2}}\left\{m_{N} \mathcal{A}_{3}^{\mathrm{v}}+\not q_{\perp} \mathcal{B}_{3}^{\mathrm{v}}\right\} N^{-}(P),
\end{aligned}
$$

and similarly for $\eta_{4}$ :

$$
\begin{aligned}
T_{z}^{4, \mathrm{em}} & =\frac{2(p z)^{3}}{m_{N}^{2}}\left\{m_{N} \mathcal{A}_{4}^{\mathrm{em}}+\not q_{\perp} \mathcal{B}_{4}^{\mathrm{em}}\right\} N^{-}(P), \\
T_{z}^{4, \mathrm{a}} & =\frac{2(p z)^{3}}{m_{N}^{2}}\left\{m_{N} \mathcal{A}_{4}^{\mathrm{a}}+\not q_{\perp} \mathcal{B}_{4}^{\mathrm{a}}\right\} \gamma_{5} N^{-}(P), \\
T_{z}^{4, \mathrm{v}} & =\frac{2(p z)^{3}}{m_{N}^{2}}\left\{m_{N} \mathcal{A}_{4}^{\mathrm{v}}+\not q_{\perp} \mathcal{B}_{4}^{\mathrm{v}}\right\} N^{-}(P),
\end{aligned}
$$

The elastic nucleon form factor contribution of interest corresponds to a pole term in the variable $P^{\prime 2}$. For the relevant projections we get

$$
\mathcal{A}_{1}^{\mathrm{em}}=\frac{2 \lambda_{1} F_{1}^{\mathrm{em}}}{m_{N}^{2}-P^{\prime 2}}, \quad \mathcal{B}_{1}^{\mathrm{em}}=\frac{\lambda_{1} F_{2}^{\mathrm{em}}}{m_{N}^{2}-P^{\prime 2}}, \quad \mathcal{A}_{1}^{\mathrm{a}, \mathrm{nc}}=\frac{2 \lambda_{1} G_{A}^{N C}}{m_{N}^{2}-P^{\prime 2}}, \quad \mathcal{B}_{1}^{\mathrm{a}, \mathrm{nc}}=\frac{\lambda_{1} G_{T}^{N C}}{m_{N}^{2}-P^{\prime 2}}
$$




$$
\begin{array}{ll}
\mathcal{A}_{1}^{\mathrm{v}, \mathrm{cc}}=\frac{2 \lambda_{1} F_{1}^{\mathrm{v}, \mathrm{cc}}}{m_{N}^{2}-P^{\prime 2}}, & \mathcal{B}_{1}^{\mathrm{v}, \mathrm{cc}}=\frac{\lambda_{1} F_{2}^{\mathrm{v}, \mathrm{cc}}}{m_{N}^{2}-P^{\prime 2}}, \\
\mathcal{A}_{3,4}^{\mathrm{em}}=\frac{2 f_{\mathrm{N}} F_{1}^{\mathrm{em}}}{m_{N}^{2}-P^{\prime 2}}, & \mathcal{B}_{3,4}^{\mathrm{em}}=\frac{-f_{\mathrm{N}} F_{2}^{\mathrm{em}}}{m_{N}^{2}-P^{\prime 2}}, \\
\mathcal{A}_{3,4}^{\mathrm{v}, \mathrm{cc}}=\frac{2 f_{\mathrm{N}} F_{1}^{\mathrm{v}, \mathrm{cc}}}{m_{N}^{2}-P^{\prime 2}}, & \mathcal{B}_{3,4}^{\mathrm{v}, \mathrm{cc}}=\frac{-f_{\mathrm{N}} F_{2}^{\mathrm{v}, \mathrm{cc}}}{m_{N}^{2}-P^{\prime 2}},
\end{array}
$$

Note that the pseudoscalar form factor $G_{P}$ does not contribute to the $z_{\nu} T_{\nu}$ projection because of the condition $q z=0$. It can be extracted from the LCSR for other structures, or through the relation to the axial form factor in Eq. (17) which is exact to our accuracy.

On the other hand, the correlation functions can be calculated in QCD for sufficiently large negative $P^{\prime 2}$ and $q^{2}=-Q^{2}$ in terms of nucleon DAs using the OPE. The corresponding expressions (to tree-level accuracy) are collected in Appendix A. Matching between the two representations one obtains the light-cone sum rule that relates the nucleon form factors with nucleon DAs. The precise procedure was described many times in the literature (see e.g. [35]) so we omit the technical steps. The resulting sum rules depend on two parameters: the continuum threshold $s_{0} \simeq(1.5 \mathrm{GeV})^{2}$ and Borel parameter $M^{2}$ which defines the scale at which the matching between the two representations is done. The dependence on the Borel parameter is rather weak. For definiteness, in the plots shown below we take $M^{2}=2 \mathrm{GeV}^{2}$.

\section{Results: Ioffe current}

In this section we present LCSR predictions for the nucleon form factors that are obtained using the Ioffe interpolating current $\eta_{1}$ for the proton. The form factors are plotted in the range of the momentum transfers $1 \leq Q^{2} \leq 10 \mathrm{GeV}^{2}$; for smaller $Q^{2}$ our approach is not applicable, for larger $Q^{2}$ we expect that radiative corrections to the sum rules (that include in particular the usual pQCD contribution) will become dominant. The calculations are done using two representative sets of nucleon distribution amplitudes: asymptotic DAs (solid curves) and including the corrections estimated using QCD sum rules (dashed curves), see Appendices B,C,D for the definitions. At this stage we do not attempt to fit the form factors by tuning the parameters of DAs, the difference between the solid and the dashed curves gives more or less the range of form factor values that can be obtained

$$
\begin{gathered}
\mathcal{A}_{1}^{\mathrm{a}, \mathrm{cc}}=\frac{2 \lambda_{1} G_{A}^{C C}}{m_{N}^{2}-P^{\prime 2}}, \quad \mathcal{B}_{1}^{\mathrm{a}, \mathrm{cc}}=\frac{\lambda_{1} G_{T}^{C C}}{m_{N}^{2}-P^{\prime 2}} \\
\mathcal{A}_{3,4}^{\mathrm{a}, \mathrm{nc}}=\frac{-2 f_{\mathrm{N}} G_{A}^{N C}}{m_{N}^{2}-P^{\prime 2}}, \quad \mathcal{B}_{3,4}^{\mathrm{a}, \mathrm{nc}}=\frac{f_{\mathrm{N}} G_{T}^{N C}}{m_{N}^{2}-P^{\prime 2}} \\
\mathcal{A}_{3,4}^{\mathrm{a}, \mathrm{cc}}=\frac{-2 f_{\mathrm{N}} G_{A}^{C C}}{m_{N}^{2}-P^{\prime 2}}, \quad \mathcal{B}_{3,4}^{\mathrm{a}, \mathrm{cc}}=\frac{f_{\mathrm{N}} G_{T}^{C C}}{m_{N}^{2}-P^{\prime 2}},
\end{gathered}
$$

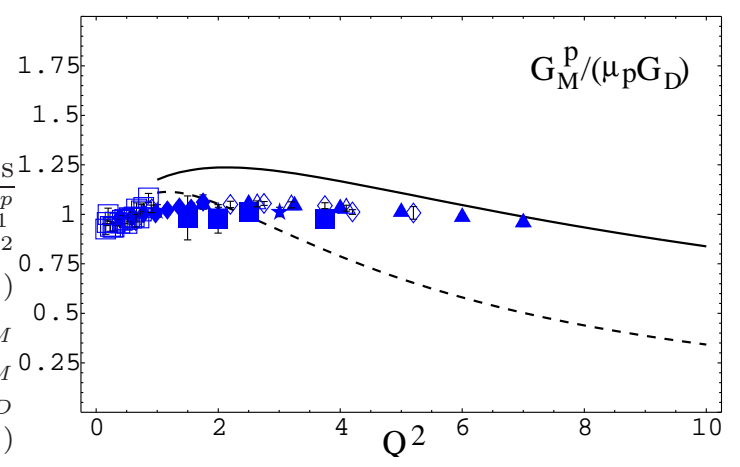

FIG. 3: LCSR prediction (Ioffe current) for the magnetic form factor of the proton normalized to the dipole form factor $G_{M}^{p} /\left(\mu_{p} G_{D}\right)$. The data points: $\star$ : SLAC 1994 [48]; $\mathbf{\Delta}:$ SLAC 1994 [49]; 口: SLAC 1970 [50]*; ४: Bonn $1971[51]^{*} ; \square$ : Stanford $1966[52]^{*} ; \diamond$ : JLab 2004 54]; $\triangle$ : JLab 2005 55]. (*: Data actually taken from [53]).

with the DAs of "reasonable" shape.

The prediction for the proton magnetic form factor normalized to the dipole form factor, $G_{M}^{p} /\left(\mu_{p} G_{D}\right)$, where

$$
G_{D}=\left(1+Q^{2} / 0.71 \mathrm{GeV}^{2}\right)^{2},
$$

is shown in Fig. B] Both the $Q^{2}$ dependence and the magnitude of the form factor is reproduced rather well, especially if using asymptotic DAs.

The result for the ratio of the proton electric form factor to the magnetic for factor, $\mu_{p} G_{E}^{p} / G_{M}^{p}$,

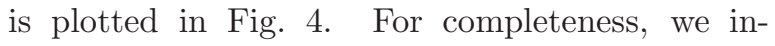
clude on this plot the data obtained both via Rosenbluth separation and the polarization transfer techniques, although the former one is most likely flawed. Most interestingly, this ratio appears to be very sensitive to the shape of nucleon DAs. Whereas the experimental data obtained via Rosenbluth separation could nicely be described by asymptotic DAs alone, the polarization transfer data require considerable corrections.

The LCSR predictions for the neutron are shown in Fig. [5 and Fig. [6 for the magnetic and the electric form factors, respectively. In this case, again, the magnetic form factor is described rea- 


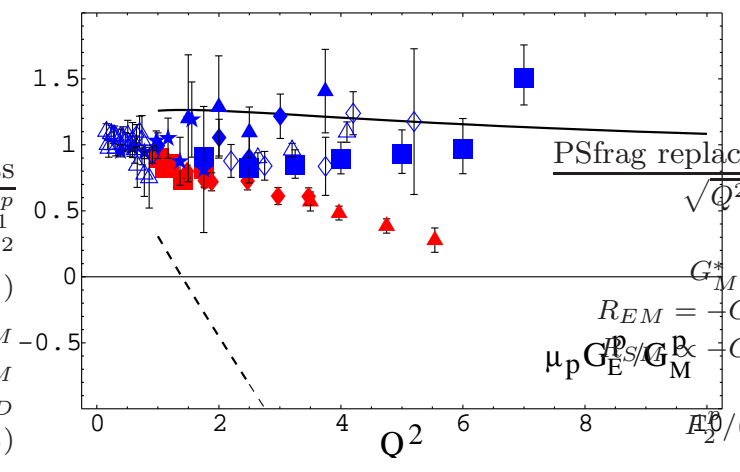

FIG. 4: LCSR prediction (Ioffe current) for the electric form factor of the proton normalized to the magnetic form factor: $\mu_{p} G_{E}^{p} / G_{M}^{p}$. The data points shown in red are obtained via Polarization transfer: $\Delta$ : Jefferson LAB 2002 [72]; ㅁ: Jefferson LAB 2001 [71]; son LAB 2000 70]; The data points shown in blue are obtained via Rosenbluth separation: घ: SLAC 1994 49]; $\diamond:$ SLAC 1994 [48]; $\mathbf{\Delta}$ : SLAC 1970 50] *; $\star$ : Bonn $1971[51]^{*} ; \square$ : Stanford $1966[52]^{*} ; \diamond$ : JLab 2004 54]; $\triangle$ : JLab 2005 [55]. (*: Data actually taken from 53]). (Color identification refers to the online version)

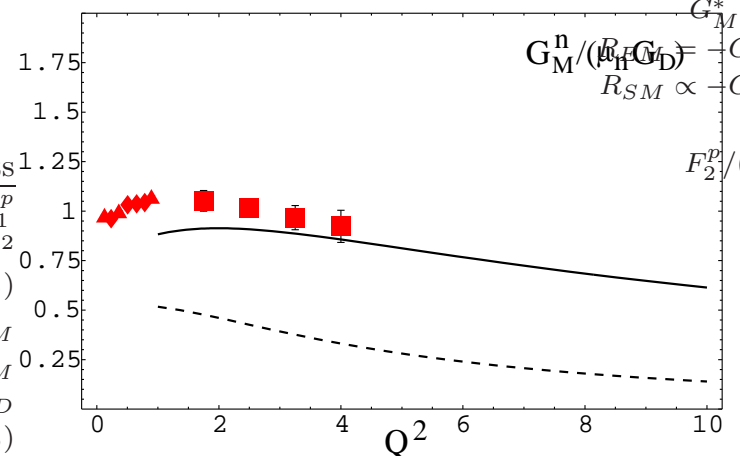

FIG. 5: LCSR prediction (Ioffe current) for the magnetic form factor of the neutron normalized to the dipole form factor $G_{M}^{n} /\left(\mu_{n} G_{D}\right)$. The data points: SLAC 1993 [56]; $\mathbf{\Delta}$ : Mainz 2002 [57]; ४: Mainz 1998 [58].

sonably well by asymptotic DAs, while the magnitude and even the sign of the electric form factor depends on their shape. Further, the LCSR prediction for the axial form factor of the proton $G_{A}^{C C}$ normalized to $G_{D}^{(a)}=1.267 /\left(1+Q^{2}\right)^{2}$ is shown in Fig. 7 Experimentally, this ratio is close to one. A more steep $Q^{2}$-dependence of the axial form factor compared to the electromagnetic ones seems to be correctly reproduced, and also the normalization agrees within $50 \%$ accuracy.

Last but not least, in Fig. 8 we show the LCSR result for the form factor $G_{T}^{C C}$ normalized to $G_{A}^{C C}$. As mentioned above, this form factor is forbidden by T-invariance so that this ratio has to be

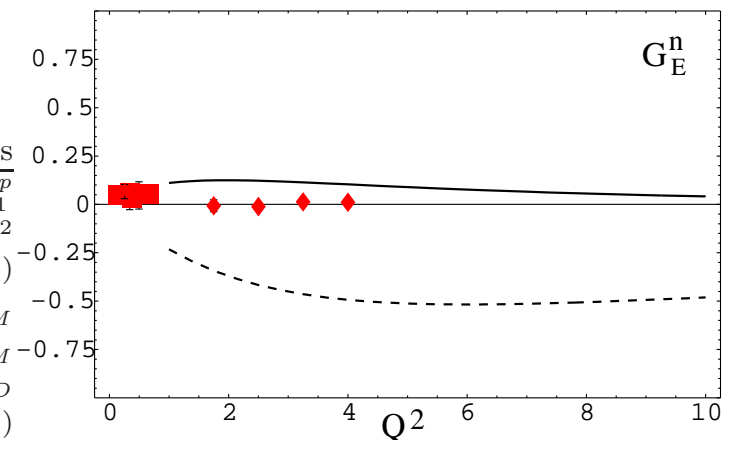

FIG. 6: LCSR prediction (Ioffe current) for the electric form factor of the neutron $G_{E}^{n}\left(Q^{2}\right)$. The data points: Red symbols: experimental values: S SLAC 1993 [56]; \: Jefferson Lab 2001 [59]; $\mathbf{0}$ : Mainz 1999 [60].

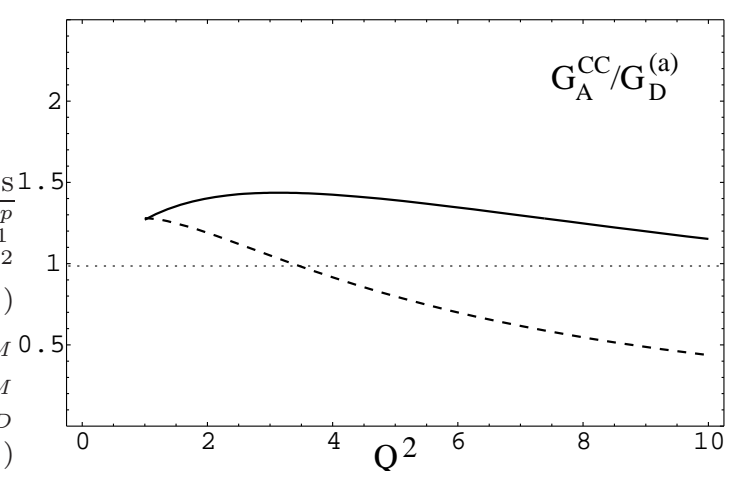

FIG. 7: LCSR prediction (Ioffe current) for the axial form factor $G_{A}^{C C}$ normalized to $G_{D}^{(a)}=g_{A} /\left(1+Q^{2}\right)^{2}$.

zero. In the LCSR approach the final and the initial state nucleons are treated differently and the T-invariance is not manifest. Smallness of $G_{T}$ is therefore an indication of how good the nucleon state is separated from the continuum by the simple duality assumption. We observe that $G_{T}^{C C} / G_{A}^{C C}$ strongly depends on the shape of the nucleon DAs. It is small and negative for asymptotic DAs but becomes positive if the DAs acquire large corrections.

\section{Results: Leading-twist currents}

\section{Checking isospin relations}

One of the main motivations for our study is to find out the optimal nucleon current for the calculation of nucleon form factors within the framework of LCSR.

In Ref. 35 the current $\eta_{4}$, Eq. (34), was used which in difference to $\eta_{3}$, Eq. (32), couples both to isospin $I=1 / 2$ and $I=3 / 2$ states. A priory, it is not obvious which current is better since the sum- 


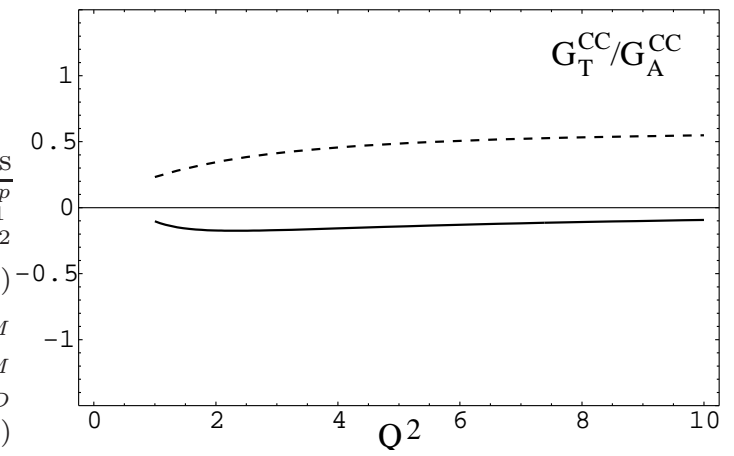

FIG. 8: LCSR prediction (Ioffe current) for the tensor form factor $G_{T}^{C C}$ normalized to $G_{A}^{C C}$.

mation over quantum numbers may improve the accuracy of the duality approximation for the continuum and also it usually suppresses poorly known contributions of higher-dimension(twist) operators. The new observation of this work is that this argumentation can be tested by checking the isospin relations e.g. for the vector current:

$$
\begin{aligned}
& F_{1}^{C C}\left(Q^{2}\right)=F_{1}^{p}\left(Q^{2}\right)-F_{1}^{n}\left(Q^{2}\right), \\
& F_{2}^{C C}\left(Q^{2}\right)=F_{2}^{p}\left(Q^{2}\right)-F_{2}^{n}\left(Q^{2}\right),
\end{aligned}
$$

cf. (14). These relations are fulfilled identically if the $\eta_{3}$ (or $\eta_{1}$ ) current is used, because in this case the correlation functions (35) satisfy isospin relations by themselves. However, when using the $\eta_{4}$ current, the extracted form factors satisfy the relations in (41) only approximately, within the sum rule accuracy. In particular, their violation provides one with a direct measure of the contamination of the nucleon contribution by isospin $I=3 / 2$ states.

The results are shown in Fig. 9 for $F_{1}$ and $F_{2}$ on the two upper and two lower panels, respectively. We see that (unphysical) isospin breaking is relatively moderate in case that asymptotic nucleon DAs are chosen, but it explodes if the DAs acquire significant corrections. The situation with axial form factors proves to be similar. Since the ultimate goal of our study is to determine nucleon DAs from the comparison to the data, such a behavior presents a crucial disadvantage. We conclude that the LCSRs with the current $\eta_{4}$ do not pass the test; they are strongly contaminated with the isospin $I=3 / 2$ contributions and do not allow for any quantitative form factor determinations. Hence, hereafter we drop the $\eta_{4}$ current and only consider $\eta_{3}$ (and $\left.\eta_{1}\right)$.

\section{Nucleon form factors for $\eta_{3}$}

Here we present LCSR results for the nucleon form factors, obtained using the interpolating current current $\eta_{3}$. Electromagnetic form factors are shown in Fig. 10] and weak form factors in Fig. 11] respectively. As above, the calculations are done using asymptotic DAs (solid curves) and including the corrections estimated using QCD sum rules (dashed curves), see Appendices B,C,D for details. The agreement with the data is in general somewhat worse compared to the calculations using the Ioffe current and, most interestingly, the corrections to asymptotic DAs "work" in opposite direction. We repeat that nonzero values obtained for the tensor form factor $G_{T}$ are artifact of our approach and can be used to quantify the error estimates.

As a matter of principle, sum rules using all interpolating currents have to produce the same results. In practice it has never been the case and the optimal choice of the interpolating current is a very important part of the QCD sum rule method. In our case it is possible that the difference between predictions based on $\eta_{1}$ and $\eta_{3}$ currents will decrease when radiative corrections to the sum rules are included. Still, on the basis of information that we have now and the experience of QCD sum rule calculations with baryons in general, we believe that the Ioffe current $\eta_{1}$ provides the best option for the construction of the LCSRs. The LCSRs based on the leading twist current $\eta_{3}$ are valid and useful for making a consistency check since their structure and the relative weight of DAs of different twist is very different.

To illustrate this issue we show in Fig. 12 the LCSR results for $F_{1}^{p}$ and $F_{2}^{p}$ obtained using the interpolating current $\eta_{1}$ (upper two panels) and $\eta_{3}$ (lower two panels) and asymptotic nucleon DAs. On each plot, solid curves correspond to the sum of contributions of all twists and the dashed-dotted curves show the contribution of leading-twist DAs only, including the corresponding nucleon mass corrections. Notice that the $Q^{2}$ dependence of the leading-twist and the higher-twist contributions is almost the same. This is to be expected, since higher-twist corrections to the sum rules are only suppressed by a power of the Borel parameter, not a power of $Q^{2}$. On the other hand, the relative weight of the leading-twist and the highertwist terms depends strongly on the current: E.g. for Ioffe current $F_{1}$ is almost entirely higher-twist, whereas for the $\eta_{3}$ current the leading-twist contribution is dominant. Also for $F_{2}$ the sum rules based on the $\eta_{3}$ current are more sensitive to the 

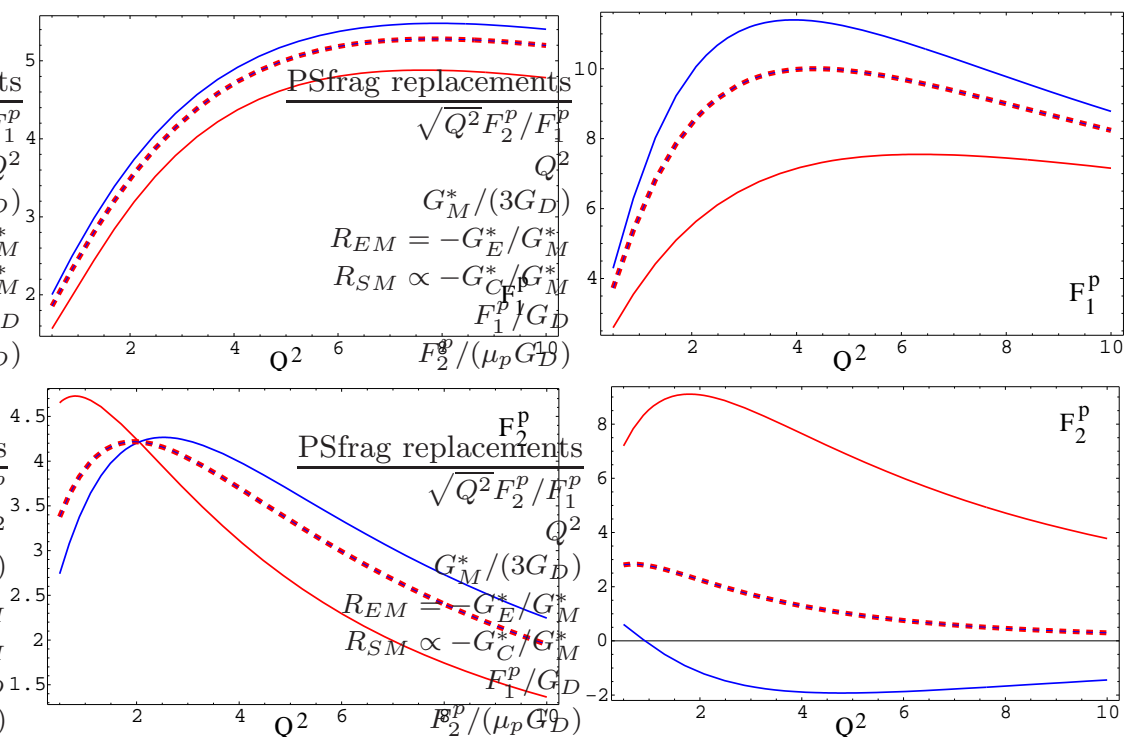

FIG. 9: Test of the isospin relations for the form factor $F_{1}$ (upper panels) and $F_{2}$ (lower panels) using the asymptotic form of the nucleon DAs (left panels) and including corrections estimated from QCD sum rules (right panels). The red (lower) and the blue (upper) solid curves show the r.h.s and the l.h.s. of the relation in 41), obtained from the LCSRs using the current $\eta_{4}$ and normalized to the dipole form factor $G_{D}$. The blue-red thick dashed curves shows the LCSR result obtained with the $\eta_{3}$ current, in which case the isospin relation is satisfied identically. (Color identification refers to the online version.)

leading-twist DAs and may be more useful to obtain restrictions on the corresponding parameters. We expect that such differences will be moderated upon inclusion of the radiative corrections to the sum rules. However, the Ioffe current-based sum rules will most likely still provide higher accuracy for the form factors.

\section{E. A model for the nucleon distribution amplitudes}

The nucleon DAs provide the principal nonperturbative input to the LCSRs. As we have seen, in many cases experimental data are in between the LCSR calculations that asymptotic and QCD sum rule-based DAs. This suggests that a good description of the data is possible by tuning the parameters of the DAs. As a demonstration, we present here the results obtained using a simple model in which the deviation from the asymptotic DAs is taken to be one third of that suggested by the QCD sum rule estimates.

The corresponding parameters are:

$$
\begin{aligned}
& A_{1}^{u}=0.13, \quad V_{1}^{d}=0.30, \\
& f_{1}^{d}=0.33, \quad f_{1}^{u}=0.09, \quad f_{2}^{d}=0.25 .
\end{aligned}
$$

where the first two refer to the leading twist-3 and the rest correspond to twist- 4 . These values are not unreasonable, since QCD sum rules are known to overestimate the matrix elements of higher conformal spin operators, and we just made the simplest assumption that all sum rule results have to be rescaled by the same factor. Our leading-twist parameter $V_{1}$ is very close to the phenomenological Bolz-Kroll model [93]; $A_{1}$ is somewhat bigger but the dependence of the leading-order sum rules on this parameter is weak. To this accuracy, the sum rules also do not depend on the parameters $\lambda_{2}$ and $f_{2}^{d}$; this dependence is present, however, in the transition form factors like $\gamma^{*} N \rightarrow \Delta$.

The calculations using this model are shown by solid curves for the electromagnetic form factors in Fig. 13 and weak form factors in Fig. 14 respectively. In addition, in Fig. 15 we plot the corresponding $\sqrt{Q^{2}} F_{2}^{p} / F_{1}^{p}$ ratio. On the same plots we show by the dotted curves the effect of the variation of the couplings ratio $f_{N} / \lambda_{1}$ (see Eqs. (33) within a conservative $30 \%$ error range. This ratio determines the overall normalization of the leading-twist DAs compared to higher twist so that the sensitivity to $f_{N} / \lambda_{1}$ is a good indication of the relative size of the leading-twist contributions to the LCSRs.

One sees that the experimental data on the electromagnetic form factors are reproduced very well, and, most welcome, the unphysical tensor form factor $G_{T}$ becomes consistent with zero. Also for the axial form factor there is a good agreement, both 

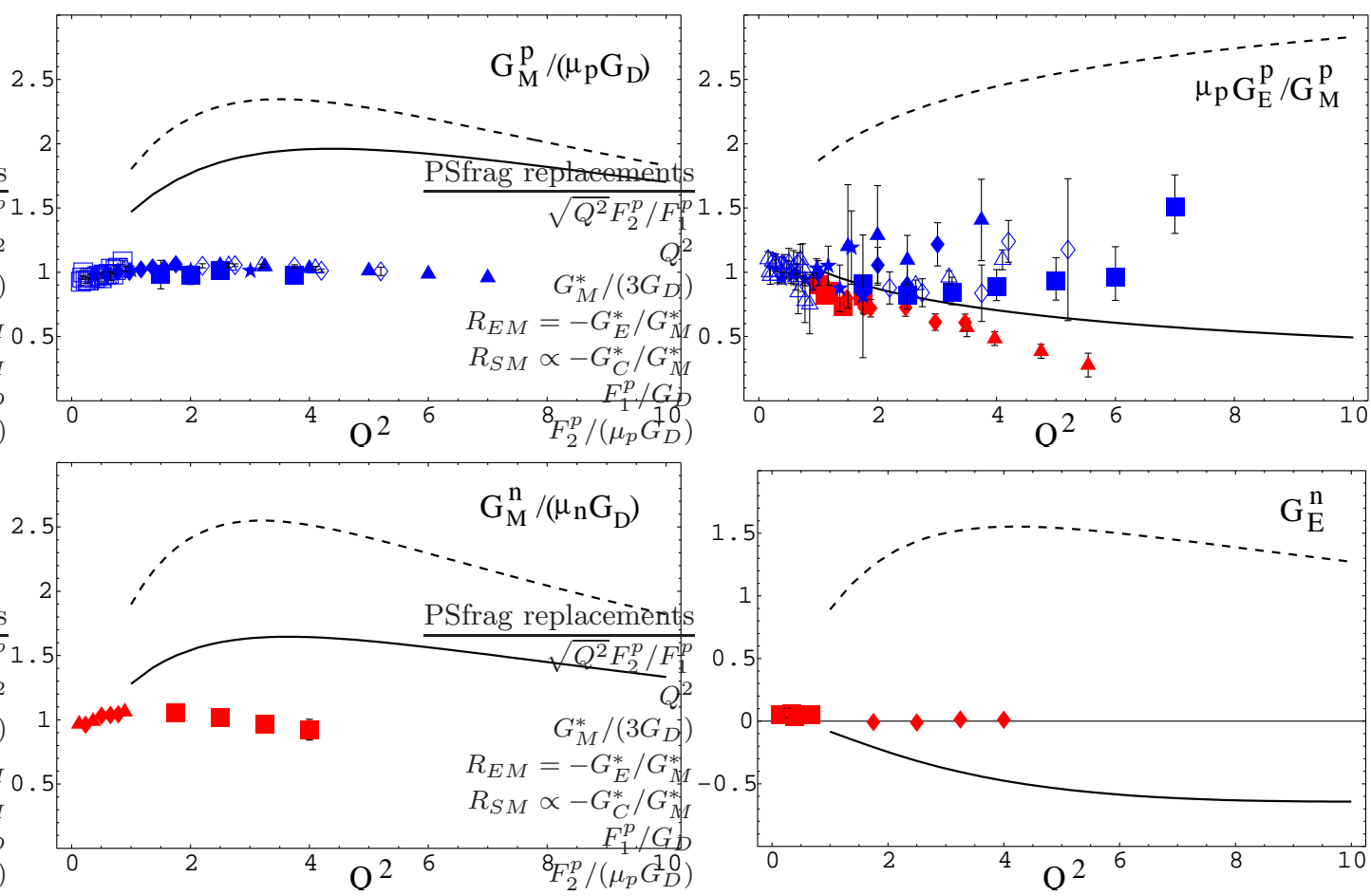

FIG. 10: LCSR results for the electromagnetic form factors of the nucleon, obtained using the leading-twist-3 interpolating current $\eta_{3}$. Identification of the curves and the data points is the same as in Figs. 3, 6
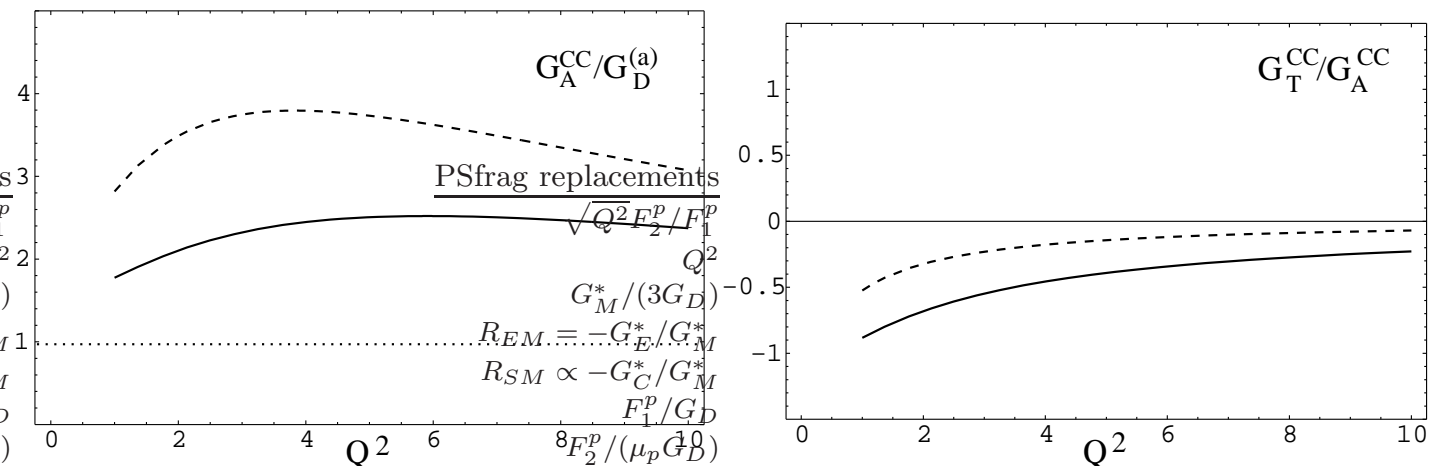

FIG. 11: LCSR results for the axial form factor of the proton $G_{A}^{C C}$ normalized to $G_{D}^{(a)}=g_{A} /\left(1+Q^{2}\right)^{2}($ left panel) and tensor form factor $G_{T}^{C C}$ normalized $G_{A}^{C C}$ (right panel), obtained using the leading-twist-3 interpolating current $\eta_{3}$.

in shape and normalization.

Last but not least, we can use the same set of DAs to calculate the $\gamma^{*} N \rightarrow \Delta$ transition form factors within the LCSR approach, following Ref. 46. The results are shown in Fig. [16] In this case we also get a much better agreement with the experimental data on the electric form factor compared to the calculations that use asymptotic or sum rulebased DAs.

We should warn that the model in Eq. (42) is not based on any systematic attempt to fit the data and in fact we believe that such any fitting would be premature before the radiative corrections to the LCSR are calculated. In addition, one has to take into account the scale dependence of the parameters of the DAs and study in more detail the dependence of the sum rules on the Borel parameter. Still, the very possibility to describe many different form factors using the same set of DAs is nontrivial and indicates the selfconsistency of our approach. The true parameters of the DAs are probably not far from the numbers quoted in Eq. (42), although at this stage we cannot give any error estimates. 

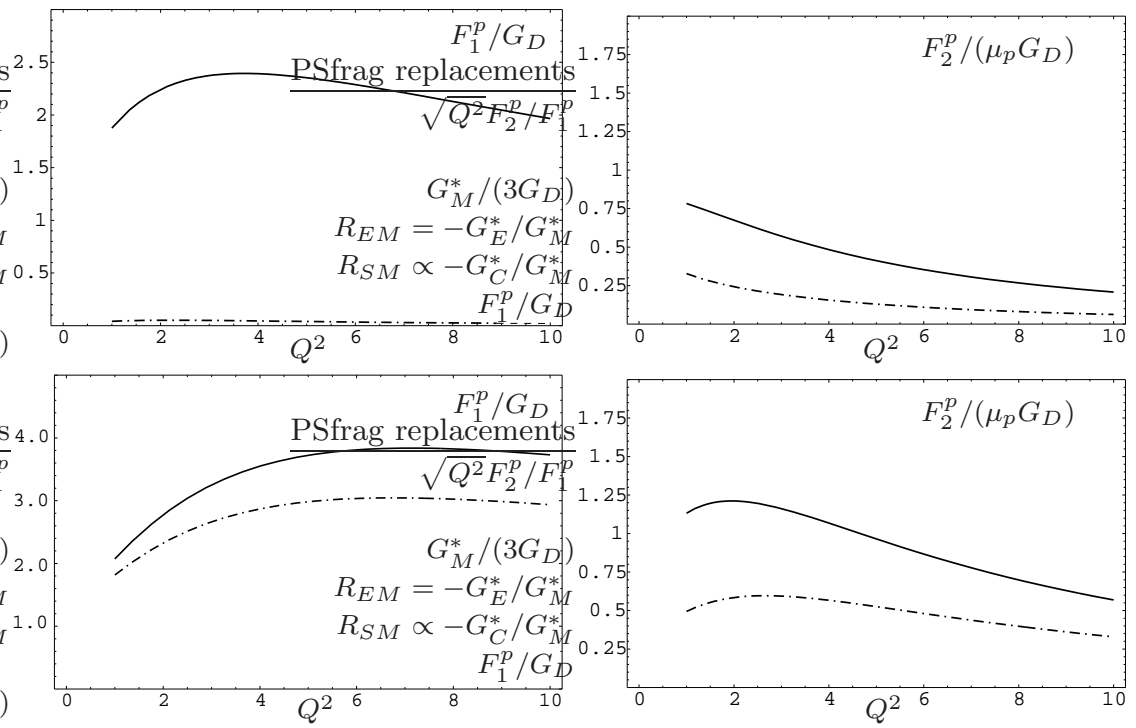

FIG. 12: LCSR results (solid curves) for the electromagnetic form factors of the nucleon, $F_{1}^{p}$ (left) and $F_{2}^{p}$ (right) obtained using the interpolating current $\eta_{1}$ (upper two panels) and $\eta_{3}$ (lower two panels) and asymptotic nucleon DAs. On each plot, the dashed-dotted curves show the contribution of leading-twist DAs only, including the corresponding nucleon mass corrections.
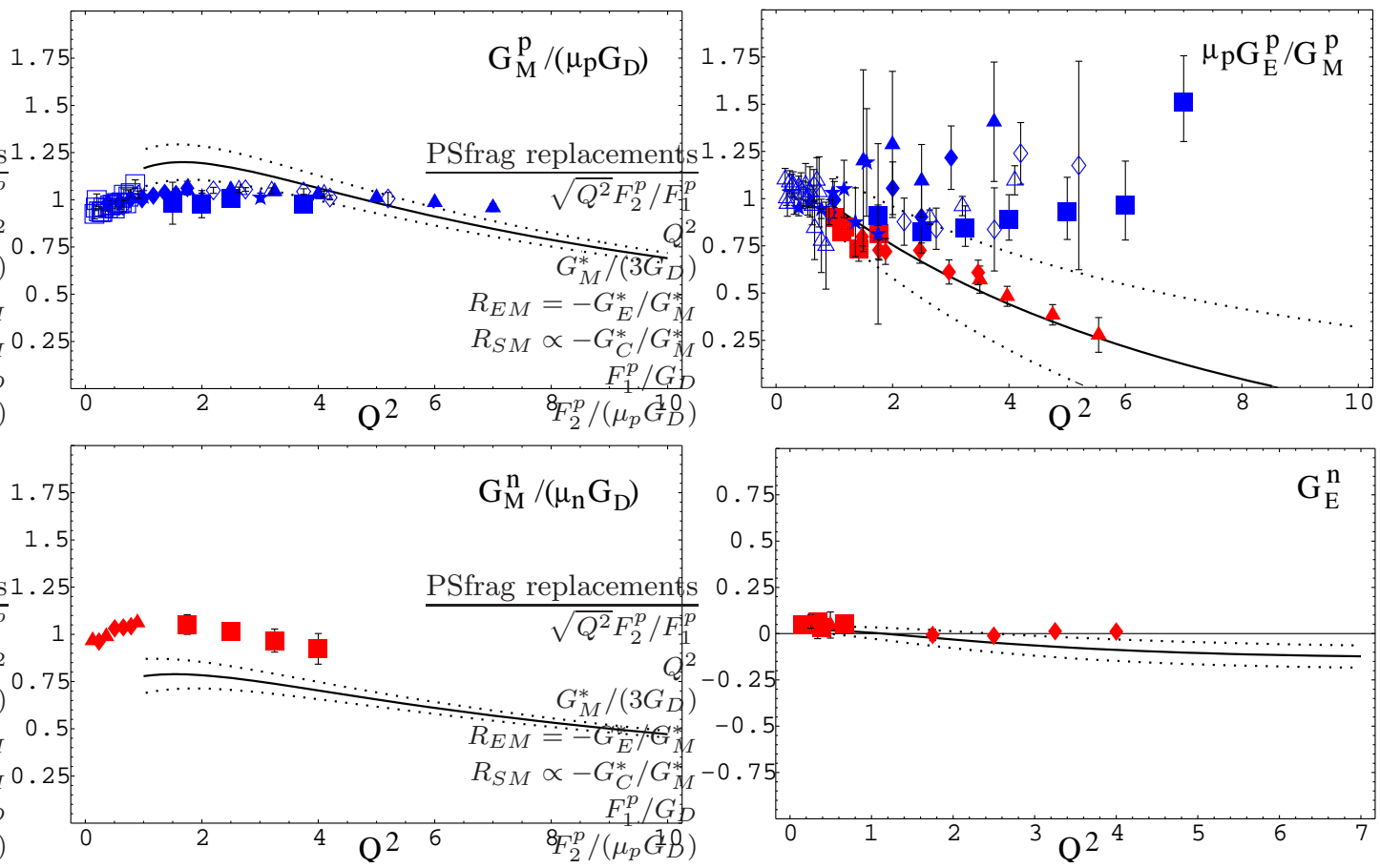

FIG. 13: LCSR results (solid curves) for the electromagnetic form factors of the nucleon, obtained using the model of the nucleon DAs (42) and the Ioffe current $\eta_{1}$. The dotted curves show the effect of the variation of the ratio $f_{N} / \lambda_{1}$ by $30 \%$. Identification of the data points is the same as in Figs. 36

\section{CONCLUSIONS}

Following the proposal in Ref. [35] we have made a comprehensive study of leading order light-cone sum rules for the electromagnetic and weak nucleon form factors. We presented detailed results that are obtained using different interpolating currents for the nucleon and argue that the Ioffe current appears to be the optimal. We make an update of the QCD sum rule estimates of the shape parame- 

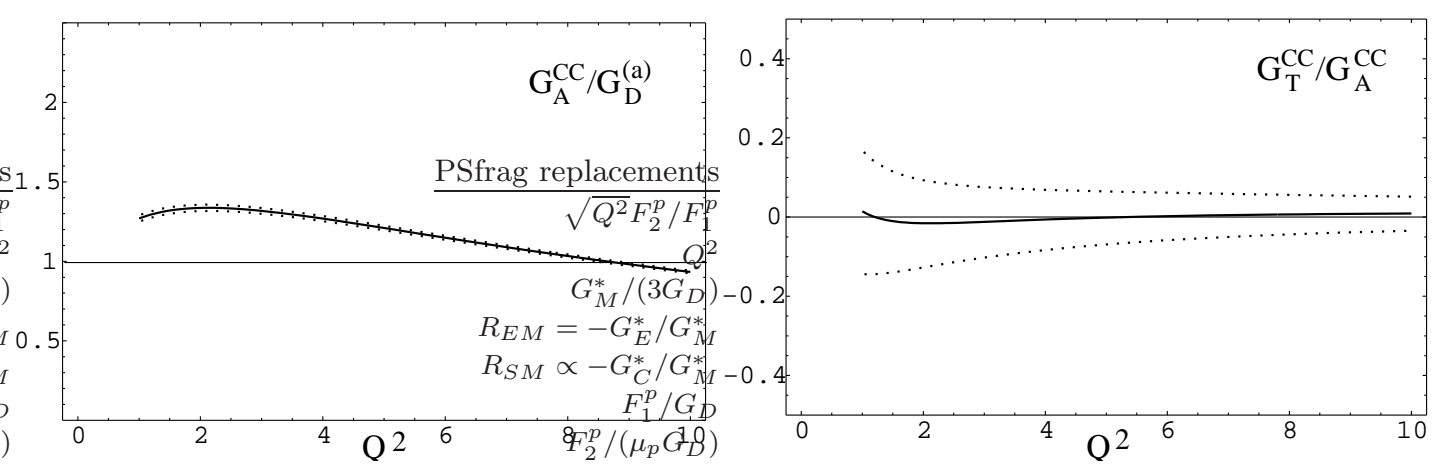

FIG. 14: LCSR results (solid curves) for the axial form factor of the proton $G_{A}^{C C}$ normalized to $G_{D}^{(a)}=g_{A} /\left(1+Q^{2}\right)^{2}$ (left panel) and tensor form factor $G_{T}^{C C}$ normalized to $G_{A}^{C C}$ (right panel), obtained using obtained using the model of the nucleon DAs (42) and the Ioffe current $\eta_{1}$. The dotted curves show the effect of the variation of the ratio $f_{N} / \lambda_{1}$ by $30 \%$.

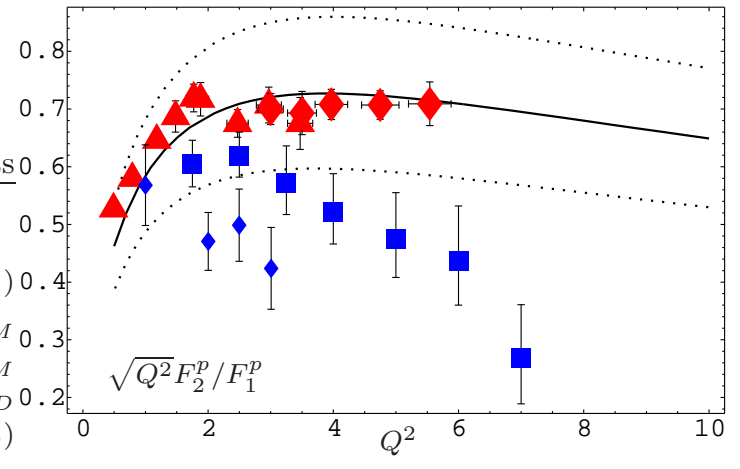

FIG. 15: LCSR results (solid curves) for the ratio $\sqrt{Q^{2}} F_{2}^{p} / F_{1}^{p}$ obtained using the model of the nucleon DAs (42) and the Ioffe current $\eta_{1}$. Data points: Red symbols: experimental values obtained via Polarization transfer: $\boldsymbol{\Delta} \wedge$ : M. Jones (private communication); Blue symbols: experimental values obtained via Rosenbluth separation: $\square$ : SLAC 1994 [49]; [48]. (Color identification refers to the online version)

ters of higher-twist DAs and also present a simple model that seems to reproduce main features of the experimental data remarkably well. In addition we included a complete summary of higher-twist DAs and some new expressions for the OPE of threequark operators which extend the results given in 35 .

Our main conclusion in this work is that the LCSR approach to baryon form factors seems to be sufficiently accurate to allow one to get a quantitative description of hard exclusive reactions with baryons. From the theory point of view, this technique is attractive because in LCSRs "soft" contributions to the form factors are calculated in terms of the same DAs that enter the pQCD calculation and there is no double counting. The asymptotic pQCD limit, in fact, formally corresponds to a part of the two-loop $\alpha_{s}^{2}$ radiative correction to the sum rules. Thus, the LCSRs provide one with the most direct relation of the hadron form factors and distribution amplitudes that is available at present, with no other nonperturbative parameters.

We remind that the sum rules considered in this work are tree-level. Further progress in this direction requires the calculation of the radiative one-loop correction to contributions of twist-3 and twist-4 operators. It would also be very valuable to have lattice evaluations for at least some of the parameters that enter the DAs, most importantly the $f_{N} / \lambda_{1}$ ratio.

\section{Acknowledgement}

We would like to thank N. Mahnke for collaboration in early stages of this work and Daowei Wang for pointing out misprints in Appendix B of 35] and for comparing the results for $\mathcal{A}_{1}^{M}$ and $\mathcal{T}_{1}^{M}$.

\section{APPENDICES}

\section{APPENDIX A: SUMMARY OF CORRELATION FUNCTIONS}

In this Appendix we present the tree-level results for the correlation functions defined in Eq. (37), (38). The correlation functions are expressed in terms of nucleon DAs that are summarized in Appendix $\mathrm{B}$ below. We use the following notations:

$$
\begin{aligned}
& \widetilde{F}\left(x_{3}\right)=\int_{1}^{x_{3}} d x_{3}^{\prime} \int_{0}^{1-x_{3}^{\prime}} d x_{1} F\left(x_{1}, 1-x_{1}-x_{3}^{\prime}, x_{3}^{\prime}\right), \\
& \widetilde{F}\left(x_{3}\right)=\int_{1}^{x_{3}} d x_{3}^{\prime} \int_{1}^{x_{3}^{\prime}} d x_{3}^{\prime \prime}
\end{aligned}
$$




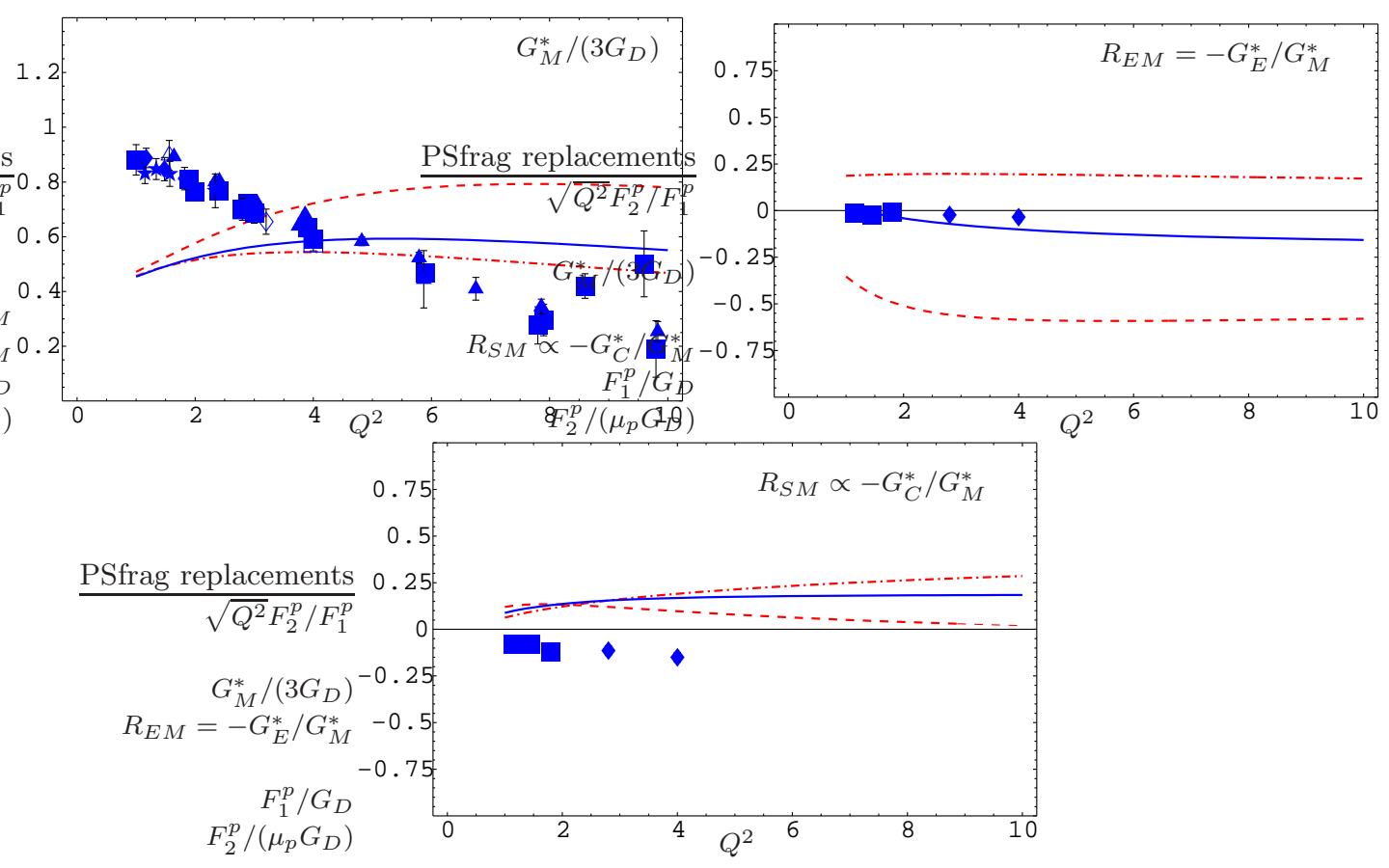

FIG. 16: $\gamma * N \rightarrow \Delta$ transition form factors in the LCSR approach [46]. The blue solid curve corresponds to the calculation using the model in Eq. (42). The red dash-dotted and dashed curves are obtained using the asymptotic and the QCD sum rule motivated DAs, respectively. For the references to the experimental data see [46]. (Color identification refers to the online version)

$$
\times \int_{0}^{1-x_{3}^{\prime \prime}} d x_{1} F\left(x_{1}, 1-x_{1}-x_{3}^{\prime \prime}, x_{3}^{\prime \prime}\right)
$$

and

$$
\begin{aligned}
\widehat{F}\left(x_{2}\right) & =\int_{1}^{x_{2}} d x_{2}^{\prime} \int_{0}^{1-x_{2}^{\prime}} d x_{1} F\left(x_{1}, x_{2}^{\prime}, 1-x_{1}-x_{2}^{\prime}\right), \\
\widehat{\widehat{F}}\left(x_{2}\right) & =\int_{1}^{x_{2}} d x_{2}^{\prime} \int_{1}^{x_{2}^{\prime}} d x_{2}^{\prime \prime} \\
& \times \int_{0}^{1-x_{2}^{\prime \prime}} d x_{1} F\left(x_{1}, x_{2}^{\prime \prime}, 1-x_{1}-x^{\prime \prime}\right), \quad(\mathrm{A} .2
\end{aligned}
$$

where $F=A, V, T$ is a generic nucleon DA that depends on the three valence quark momentum fractions, and also shorthand notations for the combinations of the DAs:

$$
\begin{aligned}
& V_{43}=V_{4}-V_{3}, \\
& V_{123}=V_{1}-V_{2}-V_{3}, \\
& V_{1345}=-2 V_{1}+V_{3}+V_{4}+2 V_{5}, \\
& V_{12345}=2 V_{1}-V_{2}-V_{3}-V_{4}-V_{5},
\end{aligned}
$$

$$
V_{123456}=-V_{1}+V_{2}+V_{3}+V_{4}+V_{5}-V_{6},(
$$

$$
\begin{aligned}
& A_{34}=A_{3}-A_{4} \\
& A_{123}=-A_{1}+A_{2}-A_{3} \\
& A_{1345}=-2 A_{1}-A_{3}-A_{4}+2 A_{5} \\
& A_{12345}=2 A_{1}-A_{2}+A_{3}+A_{4}-A_{5} \\
& A_{123456}=A_{1}-A_{2}+A_{3}+A_{4}-A_{5}+A_{6}
\end{aligned}
$$

and also

$$
\begin{aligned}
& T_{137}=T_{1}-T_{3}-T_{7}, \\
& T_{13478}=2 T_{1}-T_{3}-T_{4}-T_{7}-T_{8}, \\
& T_{134678}=T_{1}-T_{3}-T_{4}+T_{6}-T_{7}-T_{8} .
\end{aligned}
$$

In addition, in this Appendix we use

$$
q_{3} \equiv q-x_{3} P, \quad q_{2} \equiv q-x_{2} P .
$$

In this notation we obtain, for the correlation functions involving the Ioffe current $\eta_{1}$ : 


$$
\begin{aligned}
& \mathcal{A}_{1}^{\mathrm{em}}=2 e_{d} \int_{0}^{1} d x_{3}\left\{\frac{Q^{2}+q_{3}^{2}}{q_{3}^{4}} \widetilde{V}_{123}+\frac{x_{3}}{q_{3}^{2}} \int_{0}^{\bar{x}_{3}} d x_{1} V_{3}\left(x_{i}\right)+\frac{x_{3}^{2} m_{N}^{2}}{q_{3}^{4}} \widetilde{V}_{43}\right\} \\
& +2 e_{u} \int_{0}^{1} d x_{2}\left\{\frac{x_{2}}{q_{2}^{2}} \int_{0}^{\bar{x}_{2}} d x_{1}\left[-2 V_{1}+3 V_{3}+A_{3}\right]\left(x_{i}\right)-\frac{2 x_{2} m_{N}^{2}}{q_{2}^{4}} \mathcal{V}_{1}^{M(u)}+\frac{Q^{2}-q_{2}^{2}}{q_{2}^{4}} \widehat{V}_{123}\right. \\
& \left.+\frac{Q^{2}+q_{2}^{2}}{q_{2}^{4}} \widehat{A}_{123}-\frac{x_{2}^{2} m_{N}^{2}}{q_{2}^{4}}\left[\widehat{V}_{1345}-2 \widehat{V}_{43}+\widehat{A}_{34}\right]-\frac{2 x_{2} m_{N}^{2}}{q_{2}^{4}} \widehat{\widehat{V}}_{123456}\right\}, \\
& \mathcal{B}_{1}^{\mathrm{em}}=-2 e_{d} \int_{0}^{1} d x_{3}\left\{\frac{1}{q_{3}^{2}} \int_{0}^{\bar{x}_{3}} d x_{1} V_{1}\left(x_{i}\right)+\frac{m_{N}^{2}}{q_{3}^{4}} \mathcal{V}_{1}^{M(d)}-\frac{x_{3} m_{N}^{2}}{q_{3}^{4}}\left[\widetilde{V}_{123}-\widetilde{V}_{43}\right]\right\} \\
& +2 e_{u} \int_{0}^{1} d x_{2}\left\{\frac{1}{q_{2}^{2}} \int_{0}^{\bar{x}_{2}} d x_{1}\left[V_{1}+A_{1}\right]\left(x_{i}\right)+\frac{m_{N}^{2}}{q_{2}^{4}}\left[\mathcal{V}_{1}^{M(u)}+\mathcal{A}_{1}^{M(u)}\right]\right. \\
& \left.+\frac{x_{2} m_{N}^{2}}{q_{2}^{4}}\left[\widehat{V}_{1345}+\widehat{V}_{123}+\widehat{A}_{123}-2 \widehat{V}_{43}+\widehat{A}_{34}\right]\right\} \\
& \mathcal{A}_{1}^{\mathrm{a}, \mathrm{nc}}=\int_{0}^{1} d x_{3}\left\{\frac{Q^{2}+q_{3}^{2}}{q_{3}^{4}} \widetilde{V}_{123}+\frac{x_{3}}{q_{3}^{2}} \int_{0}^{\bar{x}_{3}} d x_{1} V_{3}\left(x_{i}\right)+\frac{x_{3}^{2} m_{N}^{2}}{q_{3}^{4}} \widetilde{V}_{43}\right\} \\
& +\int_{0}^{1} d x_{2}\left\{\frac{x_{2}}{q_{2}^{2}} \int_{0}^{\bar{x}_{2}} d x_{1}\left[2 A_{1}+3 A_{3}+V_{3}\right]\left(x_{i}\right)+\frac{2 x_{2} m_{N}^{2}}{q_{2}^{4}} \mathcal{A}_{1}^{M(u)}+\frac{Q^{2}-q_{2}^{2}}{q_{2}^{4}} \widehat{A}_{123}\right. \\
& \left.+\frac{Q^{2}+q_{2}^{2}}{q_{2}^{4}} \widehat{V}_{123}+\frac{x_{2}^{2} m_{N}^{2}}{q_{2}^{4}}\left[\widehat{A}_{1345}-2 \widehat{A}_{34}+\widehat{V}_{43}\right]-\frac{2 x_{2} m_{N}^{2}}{q_{2}^{4}} \widehat{\widehat{A}}_{123456}\right\}, \\
& \mathcal{B}_{1}^{\mathrm{a}, \mathrm{nc}}=\int_{0}^{1} d x_{3}\left\{\frac{1}{q_{3}^{2}} \int_{0}^{\bar{x}_{3}} d x_{1} V_{1}\left(x_{i}\right)+\frac{m_{N}^{2}}{q_{3}^{4}} \mathcal{V}_{1}^{M(d)}-\frac{x_{3} m_{N}^{2}}{q_{3}^{4}}\left[\widetilde{V}_{123}-\widetilde{V}_{43}\right]\right\} \\
& +\int_{0}^{1} d x_{2}\left\{\frac{1}{q_{2}^{2}} \int_{0}^{\bar{x}_{2}} d x_{1}\left[V_{1}+A_{1}\right]\left(x_{i}\right)+\frac{m_{N}^{2}}{q_{2}^{4}}\left[\mathcal{V}_{1}^{M(u)}+\mathcal{A}_{1}^{M(u)}\right]\right. \\
& \left.+\frac{x_{2} m_{N}^{2}}{q_{2}^{4}}\left[\widehat{A}_{1345}-\widehat{V}_{123}-\widehat{A}_{123}-2 \widehat{A}_{34}+\widehat{V}_{43}\right]\right\} \text {. }
\end{aligned}
$$

For the correlation functions involving the leading-twist current $\eta_{3}$ we get

$$
\begin{aligned}
\mathcal{A}_{3}^{\mathrm{em}}= & -\frac{4}{3} e_{u} \int_{0}^{1} \mathrm{~d} x_{2}\left\{\frac{x_{2}}{q_{2}^{2}} \int_{0}^{\bar{x}_{2}} \mathrm{~d} x_{1}\left[V_{1}+2 T_{1}\right]\left(x_{i}\right)+\frac{x_{2} m_{N}^{2}}{q_{2}^{4}}\left(\mathcal{V}_{1}^{M(u)}+2 \mathcal{T}_{1}^{M(u)}\right)-\frac{x_{2}^{2} m_{N}^{2}}{q_{2}^{4}}\left[\widehat{V}_{12345}+2 \widehat{T}_{13478}\right]\right. \\
& \left.-\frac{2 x_{2}^{3} m_{N}^{4}}{q_{2}^{6}}\left[\widehat{\widehat{V}}_{123456}-2 \widehat{\widehat{T}}_{134678}\right]\right\}-\frac{2}{3} e_{d} \int_{0}^{1} \mathrm{~d} x_{3}\left\{\left(x_{2} \rightarrow x_{3}, \widehat{F} \rightarrow \widetilde{F}\right)\right\} \\
\mathcal{B}_{3}^{\mathrm{em}}= & \frac{4}{3} e_{u} \int_{0}^{1} \mathrm{~d} x_{2}\left\{\frac{x_{2} m_{N}^{2}}{q_{2}^{4}}\left[\widehat{V}_{123}+2 \widehat{T}_{137}\right]+\frac{2 x_{2}^{2} m_{N}^{4}}{q_{2}^{6}}\left[\widehat{\widehat{V}}_{123456}-2 \widehat{\widehat{T}}_{134678}\right]\right\} \\
+ & \frac{2}{3} e_{d} \int_{0}^{1} \mathrm{~d} x_{3}\left\{\left(x_{2} \rightarrow x_{3}\right), \widehat{F} \rightarrow \widetilde{F}\right\}, \\
\mathcal{A}_{3}^{\mathrm{a}, \mathrm{nc}}= & \frac{2}{3} \int_{0}^{1} \mathrm{~d} x_{2}\left\{\frac{x_{2}}{q_{2}^{2}} \int_{0}^{\bar{x}_{2}} d x_{1}\left[A_{1}+2 T_{1}\right]\left(x_{i}\right)+\frac{x_{2} m_{N}^{2}}{q_{2}^{4}}\left[\mathcal{A}_{1}^{M(u)}+2 \mathcal{T}_{1}^{M(u)}\right]-\frac{x_{2}^{2} m_{N}^{2}}{q_{2}^{4}}\left[\widehat{A}_{12345}+2 \widehat{T}_{13478}\right]\right. \\
& \left.+\frac{2 x_{2}^{3} m_{N}^{4}}{q_{2}^{6}}\left[\widehat{\widehat{A}}_{123456}+2 \widehat{\widehat{T}}_{134678}\right]\right\}-\frac{1}{3} \int_{0}^{1} \mathrm{~d} x_{3}\left\{\frac{x_{3}}{q_{3}^{2}} \int_{0}^{\bar{x}_{3}} d x_{1}\left[V_{1}\left(x_{i}\right)-2 T_{1}\left(x_{i}\right)\right]\right.
\end{aligned}
$$




$$
\begin{aligned}
& \left.+\frac{x_{3} m_{N}^{2}}{q_{3}^{4}}\left[\mathcal{V}_{1}^{M(d)}-2 \mathcal{T}_{1}^{M(d)}\right]-\frac{x_{3}^{2} m_{N}^{2}}{q_{3}^{4}}\left[\widetilde{V}_{12345}-2 \widetilde{T}_{13478}\right]-\frac{2 x_{3}^{3} m_{N}^{4}}{q_{3}^{6}}\left[\widetilde{\widetilde{V}}_{123456}+2 \widetilde{\widetilde{T}}_{134678}\right]\right\}, \\
\mathcal{B}_{3}^{\mathrm{a}, \mathrm{nc}=}= & \frac{2}{3} \int_{0}^{1} \mathrm{~d} x_{2}\left\{\frac{x_{2} m_{N}^{2}}{q_{2}^{4}}\left[\widehat{A}_{123}-2 \widehat{T}_{137}\right]+\frac{2 x_{2}^{2} m_{N}^{4}}{q_{2}^{6}}\left[\widehat{\widehat{A}}_{123456}+2 \widehat{\widehat{T}}_{134678}\right]\right\} \\
& -\frac{1}{3} \int_{0}^{1} \mathrm{~d} x_{3}\left\{\frac{x_{3} m_{N}^{2}}{q_{3}^{4}}\left[\widetilde{V}_{123}-2 \widetilde{T}_{137}\right]+\frac{2 x_{3}^{2} m_{N}^{4}}{q_{3}^{6}}\left[\widetilde{\widetilde{V}}_{123456}+2 \widetilde{\widetilde{T}}_{134678}\right]\right\},
\end{aligned}
$$

and, finally, for the correlation functions involving $\eta_{4}$ :

$$
\begin{aligned}
& \mathcal{A}_{4}^{\mathrm{em}}=-4 e_{u} \int_{0}^{1} \mathrm{~d} x_{2}\left\{\frac{x_{2}}{q_{2}^{2}} \int_{0}^{\bar{x}_{2}} d x_{1} V_{1}\left(x_{i}\right)+\frac{x_{2} m_{N}^{2}}{q_{2}^{4}} \mathcal{V}_{1}^{M(u)}-\frac{x_{2}^{2} m_{N}^{2}}{q_{2}^{4}} \widehat{V}_{12345}-\frac{2 x_{2}^{3} m_{N}^{4}}{q_{2}^{6}} \widehat{\widehat{V}}_{123456}\right\} \\
& -2 e_{d} \int_{0}^{1} \mathrm{~d} x_{3}\left\{\left(x_{2} \rightarrow x_{3}, \widehat{F} \rightarrow \widetilde{F}\right)\right\} \\
& \mathcal{B}_{4}^{\mathrm{em}}=4 e_{u} \int_{0}^{1} \mathrm{~d} x_{2}\left\{\frac{x_{2} m_{N}^{2}}{q_{2}^{4}} \widehat{V}_{123}+\frac{2 x_{2}^{2} m_{N}^{4}}{q_{2}^{6}} \widehat{\widehat{V}}_{123456}\right\}+2 e_{d} \int_{0}^{1} \mathrm{~d} x_{3}\left\{\left(x_{2} \rightarrow x_{3}\right), \widehat{F} \rightarrow \widetilde{F}\right\}, \\
& \mathcal{A}_{4}^{\mathrm{a}, \mathrm{nc}}=2 \int_{0}^{1} \mathrm{~d} x_{2}\left\{\frac{x_{2}}{q_{2}^{2}} \int_{0}^{\bar{x}_{2}} d x_{1} A_{1}\left(x_{i}\right)+\frac{x_{2} m_{N}^{2}}{q_{2}^{4}} \mathcal{A}_{1}^{M(u)}-\frac{x_{2}^{2} m_{N}^{2}}{q_{2}^{4}} \widehat{A}_{12345}+\frac{2 x_{2}^{3} m_{N}^{4}}{q_{2}^{6}} \widehat{\widehat{A}}_{123456}\right\} \\
& -\int_{0}^{1} \mathrm{~d} x_{3}\left\{\frac{x_{3}}{q_{3}^{2}} \int_{0}^{\bar{x}_{3}} d x_{1} V_{1}\left(x_{i}\right)+\frac{x_{3} m_{N}^{2}}{q_{3}^{4}} \mathcal{V}_{1}^{M(d)}-\frac{x_{3}^{2} m_{N}^{2}}{q_{3}^{4}} \widetilde{V}_{12345}-\frac{2 x_{3}^{3} m_{N}^{4}}{q_{3}^{6}} \widetilde{\widetilde{V}}_{123456}\right\}, \\
& \mathcal{B}_{4}^{\mathrm{a}, \mathrm{nc}}=-2 \int_{0}^{1} \mathrm{~d} x_{2}\left\{\frac{x_{2} m_{N}^{2}}{q_{2}^{4}} \widehat{A}_{123}+\frac{2 x_{2}^{2} m_{N}^{4}}{q_{2}^{6}} \widehat{\widehat{A}}_{123456}\right\}-\int_{0}^{1} \mathrm{~d} x_{3}\left\{\frac{x_{3} m_{N}^{2}}{q_{3}^{4}} \widetilde{V}_{123}+\frac{2 x_{3}^{2} m_{N}^{4}}{q_{3}^{6}} \widetilde{\widetilde{V}}_{123456}\right\} \\
& \mathcal{A}_{4}^{\mathrm{v}, \mathrm{cc}}=-2 \int_{0}^{1} \mathrm{~d} x_{2}\left\{\frac{x_{2}}{q_{2}^{2}} \int_{0}^{\bar{x}_{2}} d x_{1}\left[V_{1}-A_{1}-2 T_{1}\right]\left(x_{i}\right)+\frac{x_{2} m_{N}^{2}}{q_{2}^{4}}\left[\mathcal{V}_{1}^{M(u)}-\mathcal{A}_{1}^{M(u)}-2 \mathcal{T}_{1}^{M(u)}\right]\right. \\
& \left.+\frac{x_{2}^{2} m_{N}^{2}}{q_{2}^{4}}\left[-\widehat{V}_{12345}+\widehat{A}_{12345}+2 \widehat{T}_{13478}\right]-\frac{2 x_{2}^{3} m_{N}^{4}}{q_{2}^{6}}\left[\widehat{\widehat{V}}_{123456}+\widehat{\widehat{A}}_{123456}+2 \widehat{\widehat{T}}_{134678}\right]\right\}, \\
& \mathcal{B}_{4}^{\mathrm{v}, \mathrm{cc}}=2 \int_{0}^{1} \mathrm{~d} x_{2}\left\{\frac{x_{2} m_{N}^{2}}{q_{2}^{4}}\left[\widehat{V}_{123}+\widehat{A}_{123}+2 \widehat{T}_{137}\right]+\frac{2 x_{2}^{2} m_{N}^{4}}{q_{2}^{6}}\left[\widehat{\widehat{V}}_{123456}+\widehat{\widehat{A}}_{123456}+2 \widehat{\widehat{T}}_{134678}\right]\right\}, \\
& \mathcal{A}_{4}^{\mathrm{a}, \mathrm{cc}}=2 \int_{0}^{1} \mathrm{~d} x_{2}\left\{\frac{x_{2}}{q_{2}^{2}} \int_{0}^{\bar{x}_{2}} d x_{1}\left[V_{1}-A_{1}+2 T_{1}\right]\left(x_{i}\right)+\frac{x_{2} m_{N}^{2}}{q_{2}^{4}}\left[\mathcal{V}_{1}^{M(u)}-\mathcal{A}_{1}^{M(u)}+2 \mathcal{T}_{1}^{M(u)}\right]\right. \\
& \left.+\frac{x_{2}^{2} m_{N}^{2}}{q_{2}^{4}}\left[-\widehat{V}_{12345}+\widehat{A}_{12345}-2 \widehat{T}_{13478}\right]-\frac{2 x_{2}^{3} m_{N}^{4}}{q_{2}^{6}}\left[\widehat{\widehat{V}}_{123456}+\widehat{\widehat{A}}_{123456}-2 \widehat{\widehat{T}}_{134678}\right]\right\}, \\
& \mathcal{B}_{4}^{\mathrm{a}, \mathrm{cc}}=2 \int_{0}^{1} \mathrm{~d} x_{2}\left\{\frac{x_{2} m_{N}^{2}}{q_{2}^{4}}\left[\widehat{V}_{123}+\widehat{A}_{123}-2 \widehat{T}_{137}\right]+\frac{2 x_{2}^{2} m_{N}^{4}}{q_{2}^{6}}\left[\widehat{\widehat{V}}_{123456}+\widehat{\widehat{A}}_{123456}-2 \widehat{\widehat{T}}_{134678}\right]\right\} \text {. }
\end{aligned}
$$

In all expressions the functions with a "tilde" and a "hat" have $x_{3}$ and $x_{2}$ as an argument, respectively, cf. (A.1), A.2). Also, in the terms involving two integrations over the momentum fractions, the remaining momentum fraction is replaced by using $x_{1}+x_{2}+x_{3}=1$. The results in (A.10) agree with the corresponding expressions in [35] up to two misprints: a factor two in the $\mathcal{V}^{M(u)}$-term and the sign of the $V_{123456}$ contribution; the other expressions are new.

The answers for the neutral vector current $j_{\nu}^{\mathrm{v}, \mathrm{nc}}$ are easily obtained from the corresponding expressions for the electromagnetic current by a substitution $e_{u} \rightarrow 1 / 2$ and $e_{d} \rightarrow-1 / 2$. Also, since the currents $\eta_{1}$ and $\eta_{3}$ are pure isospin $I=1 / 2$, the correlation functions involving the flavor-changing charged currents 
$j_{\nu}^{\mathrm{v}, \mathrm{cc}}$ and $j_{\nu}^{\mathrm{a}, \mathrm{cc}}$ are given in terms of the corresponding correlation functions involving the flavor-conserving currents by exact isospin relations

$$
\mathcal{A}_{i}^{\mathrm{v}, \mathrm{cc}}=2 \mathcal{A}_{i}^{\mathrm{v}, \mathrm{nc}}, \quad \mathcal{B}_{i}^{\mathrm{v}, \mathrm{cc}}=2 \mathcal{B}_{i}^{\mathrm{v}, \mathrm{nc}}, \quad \mathcal{A}_{i}^{\mathrm{a}, \mathrm{cc}}=2 \mathcal{A}_{i}^{\mathrm{a}, \mathrm{nc}}, \quad \mathcal{B}_{i}^{\mathrm{a}, \mathrm{cc}}=2 \mathcal{B}_{i}^{\mathrm{a}, \mathrm{nc}} \quad i=1,3 .
$$

These relations are not manifest because they involve isospin relations between different nucleon DAs and provide a nontrivial check of the calculation.

The Borel transformation and the continuum subtraction are performed by using the following substitution rules:

$$
\begin{aligned}
\int \mathrm{d} x \frac{\varrho(x)}{(q-x P)^{2}}= & -\int_{0}^{1} \frac{\mathrm{d} x}{x} \frac{\varrho(x)}{\left(s-P^{\prime 2}\right)} \rightarrow-\int_{x_{0}}^{1} \frac{\mathrm{d} x}{x} \varrho(x) \exp \left(-\frac{\bar{x} Q^{2}}{x M^{2}}-\frac{\bar{x} m_{N}^{2}}{M^{2}}\right) \\
\int \mathrm{d} x \frac{\varrho(x)}{(q-x P)^{4}}= & \int_{0}^{1} \frac{\mathrm{d} x}{x^{2}} \frac{\varrho(x)}{\left(s-P^{\prime 2}\right)^{2}} \rightarrow \frac{1}{M^{2}} \int_{x_{0}}^{1} \frac{\mathrm{d} x}{x^{2}} \varrho(x) \exp \left(-\frac{\bar{x} Q^{2}}{x M^{2}}-\frac{\bar{x} m_{N}^{2}}{M^{2}}\right)+\frac{\varrho\left(x_{0}\right) e^{-s_{0} / M^{2}}}{Q^{2}+x_{0}^{2} m_{N}^{2}} \\
\int \mathrm{d} x \frac{\varrho(x)}{(q-x P)^{6}}= & -\int_{0}^{1} \frac{\mathrm{d} x}{x^{3}} \frac{\varrho(x)}{\left(s-P^{\prime 2}\right)^{3}} \rightarrow-\frac{1}{2 M^{4}} \int_{x_{0}}^{1} \frac{\mathrm{d} x}{x^{3}} \varrho(x) \exp \left(-\frac{\bar{x} Q^{2}}{x M^{2}}-\frac{\bar{x} m_{N}^{2}}{M^{2}}\right) \\
& -\frac{1}{2} \frac{\varrho\left(x_{0}\right) e^{-s_{0} / M^{2}}}{x_{0}\left(Q^{2}+x_{0}^{2} m_{N}^{2}\right) M^{2}}+\frac{1}{2} \frac{x_{0}^{2}}{Q^{2}+x_{0}^{2} m_{N}^{2}}\left[\frac{d}{d x_{0}} \frac{\varrho\left(x_{0}\right)}{x_{0}\left(Q^{2}+x_{0}^{2} m_{N}^{2}\right)}\right] e^{-s_{0} / M^{2}}
\end{aligned}
$$

where $M$ is the Borel parameter, $s=\frac{1-x}{x} Q^{2}+(1-x) m_{N}^{2}$ and $x_{0}$ is the solution of the corresponding quadratic equation for $s=s_{0}$ :

$$
x_{0}=\left[\sqrt{\left(Q^{2}+s_{0}-m_{N}^{2}\right)^{2}+4 m_{N}^{2} Q^{2}}-\left(Q^{2}+s_{0}-m_{N}^{2}\right)\right] /\left(2 m_{N}^{2}\right) .
$$

The contributions $\sim e^{-s_{0} / M^{2}}$ in Eq. A.15) correspond to the "surface terms" arising from successive partial integrations to reduce the power in the denominators $(q-x P)^{2 N}=\left(s-P^{\prime 2}\right)^{2 N}(-x)^{2 N}$ with $N>1$ to the usual dispersion representation with the denominator $\sim\left(s-P^{\prime 2}\right)$. Without continuum subtraction, i.e. in the limit $s_{0} \rightarrow \infty$ these terms vanish.

In addition, in the hadronic representation for the same correlation functions one has to make the substitution

$$
\frac{1}{m_{N}^{2}-P^{\prime 2}} \rightarrow e^{-m_{N}^{2} / M^{2}}
$$

As an example, we present here the final sum rules for $F_{1}^{p}, F_{2}^{p}$ and $G_{A}^{\mathrm{NC}}$ obtained using the Ioffe current:

$$
\begin{aligned}
& F_{1}^{\mathrm{em}}\left(Q^{2}\right)=\frac{1}{2 \lambda_{1}}\left[\int_{x_{0}}^{1} \mathrm{~d} x\left(-\frac{\varrho_{2}^{a}(x)}{x}+\frac{\varrho_{4}^{a}(x)}{x^{2} M^{2}}\right) \exp \left(-\frac{\bar{x} Q^{2}}{x M^{2}}+\frac{x m_{N}^{2}}{M^{2}}\right)+\frac{\varrho_{4}^{a}\left(x_{0}\right) e^{-\left(s_{0}-m_{N}^{2}\right) / M^{2}}}{Q^{2}+x_{0}^{2} m_{N}^{2}}\right], \\
& F_{2}^{\mathrm{em}}\left(Q^{2}\right)=\frac{1}{\lambda_{1}}\left[\int_{x_{0}}^{1} \mathrm{~d} x\left(-\frac{\varrho_{2}^{b}(x)}{x}+\frac{\varrho_{4}^{b}(x)}{x^{2} M^{2}}\right) \exp \left(-\frac{\bar{x} Q^{2}}{x M^{2}}+\frac{x m_{N}^{2}}{M^{2}}\right)+\frac{\varrho_{4}^{b}\left(x_{0}\right) e^{-\left(s_{0}-m_{N}^{2}\right) / M^{2}}}{Q^{2}+x_{0}^{2} m_{N}^{2}}\right], \\
& G_{A}^{\mathrm{NC}}\left(Q^{2}\right)=\frac{1}{2 \lambda_{1}}\left[\int_{x_{0}}^{1} \mathrm{~d} x\left(-\frac{\varrho_{2}^{c}(x)}{x}+\frac{\varrho_{4}^{c}(x)}{x^{2} M^{2}}\right) \exp \left(-\frac{\bar{x} Q^{2}}{x M^{2}}+\frac{x m_{N}^{2}}{M^{2}}\right)+\frac{\varrho_{4}^{c}\left(x_{0}\right) e^{-\left(s_{0}-m_{N}^{2}\right) / M^{2}}}{Q^{2}+x_{0}^{2} m_{N}^{2}}\right],
\end{aligned}
$$

with

$$
\varrho_{2}^{a}(x)=2 e_{d}\left\{\widetilde{V}_{123}+x \int_{0}^{\bar{x}} d x_{1} V_{3}\left(x_{i}\right)+\right\}+2 e_{u}\left\{x \int_{0}^{\bar{x}} d x_{1}\left[-2 V_{1}+3 V_{3}+A_{3}\right]\left(x_{i}\right)-\widehat{V}_{123}+\widehat{A}_{123}\right\},
$$




$$
\begin{aligned}
& \varrho_{4}^{a}(x)= 2 e_{d}\left\{Q^{2} \widetilde{V}_{123}+x^{2} m_{N}^{2} \widetilde{V}_{43}\right\}+2 e_{u}\left\{Q^{2}\left(\widehat{V}_{123}+\widehat{A}_{123}\right)-x^{2} m_{N}^{2}\left[\widehat{V}_{1345}-2 \widehat{V}_{43}+\widehat{A}_{34}\right]\right. \\
&\left.-2 x m_{N}^{2}\left(\mathcal{V}_{1}^{M(u)}+\widehat{\widehat{V}}_{123456}\right)\right\}, \\
& \varrho_{2}^{b}(x)=-2 e_{d}\left\{\int_{0}^{\bar{x}} d x_{1} V_{1}\left(x_{i}\right)\right\}+2 e_{u}\left\{\int_{0}^{\bar{x}} d x_{1}\left[V_{1}+A_{1}\right]\left(x_{i}\right)\right\}, \\
& \varrho_{4}^{b}(x)=-2 e_{d} m_{N}^{2}\left\{\mathcal{V}_{1}^{M(d)}-x\left[\widetilde{V}_{123}-\widetilde{V}_{43}\right]\right\}+2 e_{u} m_{N}^{2}\left\{\left[\mathcal{V}_{1}^{M(u)}+\mathcal{A}_{1}^{M(u)}\right]\right. \\
&\left.+x\left[\widehat{V}_{1345}+\widehat{V}_{123}+\widehat{A}_{123}-2 \widehat{V}_{43}+\widehat{A}_{34}\right]\right\}, \\
& \varrho_{2}^{c}(x)=\left\{\widetilde{V}_{123}+x \int_{0}^{\bar{x}} d x_{1} V_{3}\left(x_{i}\right)\right\}+\left\{x \int_{0}^{\bar{x}} d x_{1}\left[2 A_{1}+3 A_{3}+V_{3}\right]\left(x_{i}\right)-\widehat{A}_{123}+\widehat{V}_{123}\right\}, \\
& \varrho_{4}^{c}(x)=\left\{Q^{2} \widetilde{V}_{123}+x^{2} m_{N}^{2} \widetilde{V}_{43}\right\}+\left\{Q^{2}\left(\widehat{A}_{123}+\widehat{V}_{123}\right)+x^{2} m_{N}^{2}\left[\widehat{A}_{1345}-2 \widehat{A}_{34}+\widehat{V}_{43}\right]\right. \\
&\left.+2 x m_{N}^{2}\left(\mathcal{A}_{1}^{M(u)}-\widehat{A}_{123456}\right)\right\} .
\end{aligned}
$$

Form factors of the neutron are obtained by the substitution $e_{u} \leftrightarrow e_{d}$.

\section{APPENDIX B: NUCLEON DISTRIBUTION AMPLITUDES}

In the following we give a summary of the three-quark distribution amplitudes from twist-3 to twist-6 as obtained in [83]. The DAs are defined by the matrix element of the renormalized three-quark operator at light-like separations

$$
\begin{aligned}
& 4\left\langle 0\left|\varepsilon^{i j k} u_{\alpha}^{i}\left(a_{1} z\right) u_{\beta}^{j}\left(a_{2} z\right) d_{\gamma}^{k}\left(a_{3} z\right)\right| P\right\rangle= \\
& =S_{1} m_{N} C_{\alpha \beta}\left(\gamma_{5} N^{+}\right)_{\gamma}+S_{2} m_{N} C_{\alpha \beta}\left(\gamma_{5} N^{-}\right)_{\gamma}+P_{1} m_{N}\left(\gamma_{5} C\right)_{\alpha \beta} N_{\gamma}^{+}+P_{2} m_{N}\left(\gamma_{5} C\right)_{\alpha \beta} N_{\gamma}^{-} \\
& +V_{1}(\not \not C)_{\alpha \beta}\left(\gamma_{5} N^{+}\right)_{\gamma}+V_{2}(\not \not C)_{\alpha \beta}\left(\gamma_{5} N^{-}\right)_{\gamma}+\frac{V_{3}}{2} m_{N}\left(\gamma_{\perp} C\right)_{\alpha \beta}\left(\gamma^{\perp} \gamma_{5} N^{+}\right)_{\gamma} \\
& +\frac{V_{4}}{2} m_{N}\left(\gamma_{\perp} C\right)_{\alpha \beta}\left(\gamma^{\perp} \gamma_{5} N^{-}\right)_{\gamma}+V_{5} \frac{m_{N}^{2}}{2 p z}(\not C)_{\alpha \beta}\left(\gamma_{5} N^{+}\right)_{\gamma}+\frac{m_{N}^{2}}{2 p z} V_{6}(\not C)_{\alpha \beta}\left(\gamma_{5} N^{-}\right)_{\gamma} \\
& +A_{1}\left(\not p \gamma_{5} C\right)_{\alpha \beta} N_{\gamma}^{+}+A_{2}\left(\not \partial \gamma_{5} C\right)_{\alpha \beta} N_{\gamma}^{-}+\frac{A_{3}}{2} m_{N}\left(\gamma_{\perp} \gamma_{5} C\right)_{\alpha \beta}\left(\gamma^{\perp} N^{+}\right)_{\gamma} \\
& +\frac{A_{4}}{2} m_{N}\left(\gamma_{\perp} \gamma_{5} C\right)_{\alpha \beta}\left(\gamma^{\perp} N^{-}\right)_{\gamma}+A_{5} \frac{m_{N}^{2}}{2 p z}\left(\not \gamma \gamma_{5} C\right)_{\alpha \beta} N_{\gamma}^{+}+\frac{m_{N}^{2}}{2 p z} A_{6}\left(\not z \gamma_{5} C\right)_{\alpha \beta} N_{\gamma}^{-} \\
& +T_{1}\left(i \sigma_{\perp p} C\right)_{\alpha \beta}\left(\gamma^{\perp} \gamma_{5} N^{+}\right)_{\gamma}+T_{2}\left(i \sigma_{\perp p} C\right)_{\alpha \beta}\left(\gamma^{\perp} \gamma_{5} N^{-}\right)_{\gamma}+T_{3} \frac{m_{N}}{p z}\left(i \sigma_{p z} C\right)_{\alpha \beta}\left(\gamma_{5} N^{+}\right)_{\gamma} \\
& +T_{4} \frac{m_{N}}{p z}\left(i \sigma_{z p} C\right)_{\alpha \beta}\left(\gamma_{5} N^{-}\right)_{\gamma}+T_{5} \frac{m_{N}^{2}}{2 p z}\left(i \sigma_{\perp z} C\right)_{\alpha \beta}\left(\gamma^{\perp} \gamma_{5} N^{+}\right)_{\gamma}+\frac{m_{N}^{2}}{2 p z} T_{6}\left(i \sigma_{\perp z} C\right)_{\alpha \beta}\left(\gamma^{\perp} \gamma_{5} N^{-}\right)_{\gamma} \\
& +m_{N} \frac{T_{7}}{2}\left(\sigma_{\perp \perp^{\prime}} C\right)_{\alpha \beta}\left(\sigma^{\perp \perp^{\prime}} \gamma_{5} N^{+}\right)_{\gamma}+m_{N} \frac{T_{8}}{2}\left(\sigma_{\perp \perp^{\prime}} C\right)_{\alpha \beta}\left(\sigma^{\perp \perp^{\prime}} \gamma_{5} N^{-}\right)_{\gamma},
\end{aligned}
$$


Dirac indices and $C$ is the charge-conjugation matrix 82]. Each DA $F=V_{i}, A_{i}, T_{i}, S_{i}, P_{i}$ can be represented as

$$
F\left(a_{j}, P x\right)=\int \mathcal{D} x e^{-i P x \sum_{i} x_{i} a_{i}} F\left(x_{i}\right),
$$

where the functions $F\left(x_{i}\right)$ depend on the dimensionless variables $x_{i}, 0<x_{i}<1, \sum_{i} x_{i}=1$ which correspond to the longitudinal momentum fractions carried by the quarks inside the nucleon. The integration measure is defined as

$$
\int \mathcal{D} x=\int_{0}^{1} \mathrm{~d} x_{1} \mathrm{~d} x_{2} \mathrm{~d} x_{3} \delta\left(x_{1}+x_{2}+x_{3}-1\right) .
$$

Distribution amplitudes can be expanded in contributions of operators with a given conformal spin [83. This is convenient since operators with different spin do not mix under renormalization in one loop. More importantly, only the operators with the same spin can be related by equations of motion so that the truncation of the conformal spin expansion at a certain order produces a selfconsistent approximation. In Ref. 83] the contributions of the leading and the next-to-leading order in conformal expansion have been taken into account.

To this accuracy one obtains twist-3 DAs:

$$
\begin{aligned}
V_{1}\left(x_{i}, \mu\right)= & 120 x_{1} x_{2} x_{3}\left[\phi_{3}^{0}(\mu)+\phi_{3}^{+}(\mu)\left(1-3 x_{3}\right)\right], \\
A_{1}\left(x_{i}, \mu\right)= & 120 x_{1} x_{2} x_{3}\left(x_{2}-x_{1}\right) \phi_{3}^{-}(\mu), \\
T_{1}\left(x_{i}, \mu\right)= & 120 x_{1} x_{2} x_{3}\left[\phi_{3}^{0}(\mu)\right. \\
& \left.\left.-\frac{1}{2}\left(\phi_{3}^{+}-\phi_{3}^{-}\right)(\mu)\left(1-3 x_{3}\right)\right] . \quad \text { B. } 4\right)
\end{aligned}
$$

Twist-4 distribution amplitudes:

$$
\begin{aligned}
& V_{2}\left(x_{i}, \mu\right)=24 x_{1} x_{2}\left[\phi_{4}^{0}(\mu)+\phi_{4}^{+}(\mu)\left(1-5 x_{3}\right)\right], \\
& A_{2}\left(x_{i}, \mu\right)=24 x_{1} x_{2}\left(x_{2}-x_{1}\right) \phi_{4}^{-}(\mu), \\
& T_{2}\left(x_{i}, \mu\right)=24 x_{1} x_{2}\left[\xi_{4}^{0}(\mu)+\xi_{4}^{+}(\mu)\left(1-5 x_{3}\right)\right], \\
& V_{3}\left(x_{i}, \mu\right)=12 x_{3}\left[\psi_{4}^{0}(\mu)\left(1-x_{3}\right)\right. \\
& \quad+\psi_{4}^{+}(\mu)\left(1-x_{3}-10 x_{1} x_{2}\right) \\
& \left.\quad+\psi_{4}^{-}(\mu)\left(x_{1}^{2}+x_{2}^{2}-x_{3}\left(1-x_{3}\right)\right)\right], \\
& A_{3}\left(x_{i}, \mu\right)=12 x_{3}\left(x_{2}-x_{1}\right)\left[\left(\psi_{4}^{0}+\psi_{4}^{+}\right)(\mu)\right. \\
& \left.\quad+\psi_{4}^{-}(\mu)\left(1-2 x_{3}\right)\right], \\
& T_{3}\left(x_{i}, \mu\right)=6 x_{3}\left[\left(\phi_{4}^{0}+\psi_{4}^{0}+\xi_{4}^{0}\right)(\mu)\left(1-x_{3}\right)\right. \\
& \quad+\left(\phi_{4}^{+}+\psi_{4}^{+}+\xi_{4}^{+}\right)(\mu)\left(1-x_{3}-10 x_{1} x_{2}\right) \\
& \left.\quad+\left(\phi_{4}^{-}-\psi_{4}^{-}+\xi_{4}^{-}\right)(\mu)\left(x_{1}^{2}+x_{2}^{2}-x_{3}\left(1-x_{3}\right)\right)\right],
\end{aligned}
$$

$$
\begin{aligned}
& T_{7}\left(x_{i}, \mu\right)=6 x_{3}\left[\left(\phi_{4}^{0}+\psi_{4}^{0}-\xi_{4}^{0}\right)(\mu)\left(1-x_{3}\right)\right. \\
& \quad+\left(\phi_{4}^{+}+\psi_{4}^{+}-\xi_{4}^{+}\right)(\mu)\left(1-x_{3}-10 x_{1} x_{2}\right) \\
& \left.\quad+\left(\phi_{4}^{-}-\psi_{4}^{-}-\xi_{4}^{-}\right)(\mu)\left(x_{1}^{2}+x_{2}^{2}-x_{3}\left(1-x_{3}\right)\right)\right], \\
& S_{1}\left(x_{i}, \mu\right)=6 x_{3}\left(x_{2}-x_{1}\right)\left[\left(\phi_{4}^{0}+\psi_{4}^{0}+\xi_{4}^{0}+\phi_{4}^{+}+\psi_{4}^{+}\right.\right. \\
& \left.\left.\quad+\xi_{4}^{+}\right)(\mu)+\left(\phi_{4}^{-}-\psi_{4}^{-}+\xi_{4}^{-}\right)(\mu)\left(1-2 x_{3}\right)\right], \\
& P_{1}\left(x_{i}, \mu\right)=6 x_{3}\left(x_{1}-x_{2}\right)\left[\left(\phi_{4}^{0}+\psi_{4}^{0}-\xi_{4}^{0}+\phi_{4}^{+}+\psi_{4}^{+}\right.\right. \\
& \left.\left.\quad-\xi_{4}^{+}\right)(\mu)+\left(\phi_{4}^{-}-\psi_{4}^{-}-\xi_{4}^{-}\right)(\mu)\left(1-2 x_{3}\right)\right] .
\end{aligned}
$$

Note that $T_{3}$ and $T_{7}$ differ only in the sign of the $\xi$-contributions, while $P_{1}$ and $S_{1}$ differ only in the sign of the $\phi$ - and $\psi$-contributions.

Twist-5 distribution amplitudes:

$$
\begin{aligned}
& V_{4}\left(x_{i}, \mu\right)=3\left[\psi_{5}^{0}(\mu)\left(1-x_{3}\right)\right. \\
& +\psi_{5}^{+}(\mu)\left(1-x_{3}-2\left(x_{1}^{2}+x_{2}^{2}\right)\right) \\
& \left.+\psi_{5}^{-}(\mu)\left(2 x_{1} x_{2}-x_{3}\left(1-x_{3}\right)\right)\right], \\
& A_{4}\left(x_{i}, \mu\right)=3\left(x_{2}-x_{1}\right)\left[-\psi_{5}^{0}(\mu)+\psi_{5}^{+}(\mu)\left(1-2 x_{3}\right)\right. \\
& \left.+\psi_{5}^{-}(\mu) x_{3}\right], \\
& T_{4}\left(x_{i}, \mu\right)=\frac{3}{2}\left[\left(\phi_{5}^{0}+\psi_{5}^{0}+\xi_{5}^{0}\right)(\mu)\left(1-x_{3}\right)\right. \\
& +\left(\phi_{5}^{+}+\psi_{5}^{+}+\xi_{5}^{+}\right)(\mu)\left(1-x_{3}-2\left(x_{1}^{2}+x_{2}^{2}\right)\right) \\
& \left.+\left(\phi_{5}^{-}-\psi_{5}^{-}+\xi_{5}^{-}\right)(\mu)\left(2 x_{1} x_{2}-x_{3}\left(1-x_{3}\right)\right)\right], \\
& T_{8}\left(x_{i}, \mu\right)=\frac{3}{2}\left[\left(\phi_{5}^{0}+\psi_{5}^{0}-\xi_{5}^{0}\right)(\mu)\left(1-x_{3}\right)\right. \\
& +\left(\phi_{5}^{+}+\psi_{5}^{+}-\xi_{5}^{+}\right)(\mu)\left(1-x_{3}-2\left(x_{1}^{2}+x_{2}^{2}\right)\right) \\
& \left.+\left(\phi_{5}^{-}-\psi_{5}^{-}-\xi_{5}^{-}\right)(\mu)\left(2 x_{1} x_{2}-x_{3}\left(1-x_{3}\right)\right)\right], \\
& V_{5}\left(x_{i}, \mu\right)=6 x_{3}\left[\phi_{5}^{0}(\mu)+\phi_{5}^{+}(\mu)\left(1-2 x_{3}\right)\right], \\
& A_{5}\left(x_{i}, \mu\right)=6 x_{3}\left(x_{2}-x_{1}\right) \phi_{5}^{-}(\mu) \text {, } \\
& T_{5}\left(x_{i}, \mu\right)=6 x_{3}\left[\xi_{5}^{0}(\mu)+\xi_{5}^{+}(\mu)\left(1-2 x_{3}\right)\right], \\
& S_{2}\left(x_{i}, \mu\right)=\frac{3}{2}\left(x_{2}-x_{1}\right)\left[-\left(\phi_{5}^{0}+\psi_{5}^{0}+\xi_{5}^{0}\right)(\mu)\right. \\
& +\left(\phi_{5}^{+}+\psi_{5}^{+}+\xi_{5}^{+}\right)(\mu)\left(1-2 x_{3}\right) \\
& \left.+\left(\phi_{5}^{-}-\psi_{5}^{-}+\xi_{5}^{-}\right)(\mu) x_{3}\right] \text {, } \\
& P_{2}\left(x_{i}, \mu\right)=\frac{3}{2}\left(x_{2}-x_{1}\right)\left[-\left(-\phi_{5}^{0}-\psi_{5}^{0}+\xi_{5}^{0}\right)(\mu)\right. \\
& +\left(-\phi_{5}^{+}-\psi_{5}^{+}+\xi_{5}^{+}\right)(\mu)\left(1-2 x_{3}\right) \\
& \left.+\left(-\phi_{5}^{-}+\psi_{5}^{-}+\xi_{5}^{-}\right)(\mu) x_{3}\right] \text {, }
\end{aligned}
$$


Note that $T_{4}$ and $T_{8}$ differ only in the sign of the $\xi$-contributions, while $P_{2}$ and $S_{2}$ differ only in the sign of the $\phi$ - and $\psi$-contributions. Note that the results for $S_{2}$ and $P_{2}$ quoted in 83] contain misprints.

Finally, twist-6 distribution amplitudes:

$$
\begin{aligned}
& V_{6}\left(x_{i}, \mu\right)=2\left[\phi_{6}^{0}(\mu)+\phi_{6}^{+}(\mu)\left(1-3 x_{3}\right)\right], \\
& A_{6}\left(x_{i}, \mu\right)=2\left(x_{2}-x_{1}\right) \phi_{6}^{-}, \\
& T_{6}\left(x_{i}, \mu\right)=2\left[\phi_{6}^{0}(\mu)-\frac{1}{2}\left(\phi_{6}^{+}-\phi_{6}^{-}\right)\left(1-3 x_{3}\right)\right] .
\end{aligned}
$$

In all cases $\mu$ is the renormalization scale.

The coefficients in the above expansions can be expressed in terms of eight non-perturbative parameters $f_{N}, \lambda_{1}, \lambda_{2}, f_{1}^{u}, f_{1}^{d}, f_{2}^{d}, A_{1}^{u}, V_{1}^{d}$ which are defined in Appendix D, see also [83], section 3.2. The corresponding relations read, for the leading conformal spin:

$$
\begin{aligned}
& \phi_{3}^{0}=\phi_{6}^{0}=f_{N}, \quad \phi_{4}^{0}=\phi_{5}^{0}=\frac{1}{2}\left(f_{N}+\lambda_{1}\right), \\
& \xi_{4}^{0}=\xi_{5}^{0}=\frac{1}{6} \lambda_{2}, \psi_{4}^{0}=\psi_{5}^{0}=\frac{1}{2}\left(f_{N}-\lambda_{1}\right) .
\end{aligned}
$$

For the next-to-leading spin, for twist-3:

$$
\phi_{3}^{-}=\frac{21}{2} f_{N} A_{1}^{u}, \phi_{3}^{+}=\frac{7}{2} f_{N}\left(1-3 V_{1}^{d}\right),
$$

for twist-4:

$$
\begin{aligned}
& \phi_{4}^{+}=\frac{1}{4}\left[f_{N}\left(3-10 V_{1}^{d}\right)+\lambda_{1}\left(3-10 f_{1}^{d}\right)\right], \\
& \phi_{4}^{-}=-\frac{5}{4}\left[f_{N}\left(1-2 A_{1}^{u}\right)-\lambda_{1}\left(1-2 f_{1}^{d}-4 f_{1}^{u}\right)\right], \\
& \psi_{4}^{+}=-\frac{1}{4}\left[f_{N}\left(2+5 A_{1}^{u}-5 V_{1}^{d}\right)-\lambda_{1}\left(2-5 f_{1}^{d}-5 f_{1}^{u}\right)\right], \\
& \psi_{4}^{-}=\frac{5}{4}\left[f_{N}\left(2-A_{1}^{u}-3 V_{1}^{d}\right)-\lambda_{1}\left(2-7 f_{1}^{d}+f_{1}^{u}\right)\right], \\
& \xi_{4}^{+}=\frac{1}{16} \lambda_{2}\left(4-15 f_{2}^{d}\right), \xi_{4}^{-}=\frac{5}{16} \lambda_{2}\left(4-15 f_{2}^{d}\right),(\mathrm{B} .10)
\end{aligned}
$$

for twist-5:

$$
\begin{aligned}
\phi_{5}^{+} & =-\frac{5}{6}\left[f_{N}\left(3+4 V_{1}^{d}\right)-\lambda_{1}\left(1-4 f_{1}^{d}\right)\right], \\
\phi_{5}^{-} & =-\frac{5}{3}\left[f_{N}\left(1-2 A_{1}^{u}\right)-\lambda_{1}\left(f_{1}^{d}-f_{1}^{u}\right)\right], \\
\psi_{5}^{+} & =-\frac{5}{6}\left[f_{N}\left(5+2 A_{1}^{u}-2 V_{1}^{d}\right)-\lambda_{1}\left(1-2 f_{1}^{d}-2 f_{1}^{u}\right)\right], \\
\psi_{5}^{-} & =\frac{5}{3}\left[f_{N}\left(2-A_{1}^{u}-3 V_{1}^{d}\right)+\lambda_{1}\left(f_{1}^{d}-f_{1}^{u}\right)\right], \\
\xi_{5}^{+} & \left.=\frac{5}{36} \lambda_{2}\left(2-9 f_{2}^{d}\right), \quad \xi_{5}^{-}=-\frac{5}{4} \lambda_{2} f_{2}^{d}, \quad, \quad \mathrm{~B} .11\right)
\end{aligned}
$$

and for twist- 6 :

$$
\begin{aligned}
& \phi_{6}^{+}=\frac{1}{2}\left[f_{N}\left(1-4 V_{1}^{d}\right)-\lambda_{1}\left(1-2 f_{1}^{d}\right)\right], \\
& \phi_{6}^{-}=\frac{1}{2}\left[f_{N}\left(1+4 A_{1}^{u}\right)+\lambda_{1}\left(1-4 f_{1}^{d}-2 f_{1}^{u}\right)\right] .
\end{aligned}
$$

The normalization of all DAs is determined by three dimensionful parameters $f_{N}, \lambda_{1}, \lambda_{2}$ that are well known from the QCD sum rule literature and correspond to nucleon couplings to the existing three different three-quark local operators with the correct spin and isospin, see [84]. The numerical values (at the scale $\mu=1 \mathrm{GeV}$ ) are [83, 88]:

$$
\begin{aligned}
& f_{N}=(5.0 \pm 0.5) \cdot 10^{-3} \mathrm{GeV}^{2} \\
& \lambda_{1}=-(2.7 \pm 0.9) \cdot 10^{-2} \mathrm{GeV}^{2} \\
& \lambda_{2}=(5.4 \pm 1.9) \cdot 10^{-2} \mathrm{GeV}^{2}
\end{aligned}
$$

see also Appendix D. The remaining five parameters determine the shape of the DAs (deviation from the asymptotic form) and their values are much more controversial. Asymptotic DAs correspond to the choice

$$
\begin{aligned}
& V_{1}^{d}=\frac{1}{3}, A_{1}^{u}=0 \\
& f_{1}^{d}=\frac{3}{10}, f_{1}^{u}=\frac{1}{10}, f_{2}^{d}=\frac{4}{15} .
\end{aligned}
$$

The leading-twist-3 parameters $V_{1}^{d}$ and $A_{1}^{u}$ were calculated using QCD sum rules in Refs [88, 91, 92] with the result 92 ]

$$
\begin{aligned}
& A_{1}^{u}=0.38 \pm 0.15 \\
& V_{1}^{d}=0.23 \pm 0.03
\end{aligned}
$$

while the remaining twist-4 shape parameters $f_{1}^{d}, f_{1}^{u}, f_{2}^{d}$ were estimated by the same method in 83. In this work we reconsider the corresponding sum rules (see Appendix D) and obtain a new estimate

$$
\begin{gathered}
f_{1}^{d}=0.40 \pm 0.05, \\
f_{2}^{d}=0.22 \pm 0.05, \\
f_{1}^{u}=0.07 \pm 0.05 .
\end{gathered}
$$

The set of nucleon DAs obtained using the parameters in B.15 and B.16 is sometimes referred to as the Chernyak-Zhitnitsky-like model of the DAs.

Alternatively, there exists a phenomenological model for the leading-twist DA [93] which was obtained by modelling the soft contribution by a convolution of light-cone wave functions. This model 
is much closer to the asymptotic DA compared to the CZ-model and corresponds to the choice 93 .

$$
\begin{gathered}
A_{1}^{u}=1 / 14, \\
V_{1}^{d}=13 / 42,
\end{gathered}
$$

Estimates of the higher-twist DAs in the same tech- nique are not available.

In the main text we suggest one more model that allows one to obtain good agreement with the data on the nucleon form factors within the LCSR approach. The corresponding parameters are given in Eq. (42).

\section{APPENDIX C: OPERATOR PRODUCT EXPANSION OF THREE-QUARK CURRENTS}

In this Appendix we present the tree-level expansion of the nucleon matrix element of the three-quark operator in terms of nucleon DAs to the twist-5 accuracy. The general Lorentz decomposition reads 83]

$$
\begin{aligned}
& \left\langle 0\left|\varepsilon^{i j k} u_{\alpha}^{i}\left(a_{1} x\right) u_{\beta}^{j}\left(a_{2} x\right) d_{\gamma}^{k}\left(a_{3} x\right)\right| P\right\rangle \\
= & \mathcal{S}_{1} m_{N} C_{\alpha \beta}\left(\gamma_{5} N\right)_{\gamma}+\mathcal{S}_{2} m_{N}^{2} C_{\alpha \beta}\left(\not x \gamma_{5} N\right)_{\gamma}+\mathcal{P}_{1} m_{N}\left(\gamma_{5} C\right)_{\alpha \beta} N_{\gamma}+\mathcal{P}_{2} m_{N}^{2}\left(\gamma_{5} C\right)_{\alpha \beta}(\not x N)_{\gamma} \\
& +\left(\mathcal{V}_{1}+\frac{x^{2} m_{N}^{2}}{4} \mathcal{V}_{1}^{M}\right)(P C)_{\alpha \beta}\left(\gamma_{5} N\right)_{\gamma}+\mathcal{V}_{2} m_{N}(P C)_{\alpha \beta}\left(\not x \gamma_{5} N\right)_{\gamma}+\mathcal{V}_{3} m_{N}\left(\gamma_{\mu} C\right)_{\alpha \beta}\left(\gamma^{\mu} \gamma_{5} N\right)_{\gamma} \\
& +\mathcal{V}_{4} m_{N}^{2}(\not x C)_{\alpha \beta}\left(\gamma_{5} N\right)_{\gamma}+\mathcal{V}_{5} m_{N}^{2}\left(\gamma_{\mu} C\right)_{\alpha \beta}\left(i \sigma^{\mu \nu} x_{\nu} \gamma_{5} N\right)_{\gamma}+\mathcal{V}_{6} m_{N}^{3}(\not x C)_{\alpha \beta}\left(\not x \gamma_{5} N\right)_{\gamma} \\
& +\left(\mathcal{A}_{1}+\frac{x^{2} m_{N}^{2}}{4} \mathcal{A}_{1}^{M}\right)\left(P \gamma_{5} C\right)_{\alpha \beta} N_{\gamma}+\mathcal{A}_{2} m_{N}\left(P \gamma_{5} C\right)_{\alpha \beta}(\not x N)_{\gamma}+\mathcal{A}_{3} m_{N}\left(\gamma_{\mu} \gamma_{5} C\right)_{\alpha \beta}\left(\gamma^{\mu} N\right)_{\gamma} \\
& +\mathcal{A}_{4} m_{N}^{2}\left(\not x \gamma_{5} C\right)_{\alpha \beta} N \gamma+\mathcal{A}_{5} m_{N}^{2}\left(\gamma_{\mu} \gamma_{5} C\right)_{\alpha \beta}\left(i \sigma^{\mu \nu} x_{\nu} N\right)_{\gamma}+\mathcal{A}_{6} m_{N}^{3}\left(\not x \gamma_{5} C\right)_{\alpha \beta}(\not x N)_{\gamma} \\
& +\left(\mathcal{T}_{1}+\frac{x^{2} m_{N}^{2}}{4} \mathcal{T}_{1}^{M}\right)\left(P^{\nu} i \sigma_{\mu \nu} C\right)_{\alpha \beta}\left(\gamma^{\mu} \gamma_{5} N\right)_{\gamma}+\mathcal{T}_{2} m_{N}\left(x^{\mu} P^{\nu} i \sigma_{\mu \nu} C\right)_{\alpha \beta}\left(\gamma_{5} N\right)_{\gamma} \\
& +\mathcal{T}_{3} m_{N}\left(\sigma_{\mu \nu} C\right)_{\alpha \beta}\left(\sigma^{\mu \nu} \gamma_{5} N\right)_{\gamma}+\mathcal{T}_{4} m_{N}\left(P^{\nu} \sigma_{\mu \nu} C\right)_{\alpha \beta}\left(\sigma^{\mu \varrho} x_{\varrho} \gamma_{5} N\right)_{\gamma}+\mathcal{T}_{5} m_{N}^{2}\left(x^{\nu} i \sigma_{\mu \nu} C\right)_{\alpha \beta}\left(\gamma^{\mu} \gamma_{5} N\right)_{\gamma} \\
& +\mathcal{T}_{6} m_{N}^{2}\left(x^{\mu} P^{\nu} i \sigma_{\mu \nu} C\right)_{\alpha \beta}\left(\not x \gamma_{5} N\right)_{\gamma}+\mathcal{T}_{7} m_{N}^{2}\left(\sigma_{\mu \nu} C\right)_{\alpha \beta}\left(\sigma^{\mu \nu} \not x \gamma_{5} N\right)_{\gamma}+\mathcal{T}_{8} m_{N}^{3}\left(x^{\nu} \sigma_{\mu \nu} C\right)_{\alpha \beta}\left(\sigma^{\mu \varrho} x_{\varrho} \gamma_{5} N\right)_{\gamma} .
\end{aligned}
$$

where it is assumed that the "calligrafic" functions depend on $x^{2}$ at most logarithmically. Leaving aside the terms in $x^{2}, \mathcal{V}_{1}^{M}, \mathcal{A}_{1}^{M}$ and $\mathcal{T}_{1}^{M}$, the rest of the functions can be expressed, to the tree-level accuracy, in terms of the nucleon DAs at the renormalization scale $\mu^{2} \sim\left|1 / x^{2}\right|$ :

$\mathcal{S}_{1}=S_{1}, \quad 2 P x \mathcal{S}_{2}=S_{1}-S_{2}, \quad \mathcal{P}_{1}=P_{1}, \quad 2 P x \mathcal{P}_{2}=P_{2}-P_{1}, \quad \mathcal{V}_{1}=V_{1}, \quad 2 P x \mathcal{V}_{2}=V_{1}-V_{2}-V_{3}$,

$2 \mathcal{V}_{3}=V_{3}, \quad 4 P x \mathcal{V}_{4}=-2 V_{1}+V_{3}+V_{4}+2 V_{5}, \quad 4 P x \mathcal{V}_{5}=V_{4}-V_{3}$,

$4(P x)^{2} \mathcal{V}_{6}=-V_{1}+V_{2}+V_{3}+V_{4}+V_{5}-V_{6}, \quad \mathcal{A}_{1}=A_{1}, \quad 2 P x \mathcal{A}_{2}=-A_{1}+A_{2}-A_{3}$,

$2 \mathcal{A}_{3}=A_{3}, \quad 4 P x \mathcal{A}_{4}=-2 A_{1}-A_{3}-A_{4}+2 A_{5}, \quad 4 P x \mathcal{A}_{5}=A_{3}-A_{4}$,

$4(P x)^{2} \mathcal{A}_{6}=A_{1}-A_{2}+A_{3}+A_{4}-A_{5}+A_{6}, \quad \mathcal{T}_{1}=T_{1}, \quad 2 P x \mathcal{T}_{2}=T_{1}+T_{2}-2 T_{3}, \quad 2 \mathcal{T}_{3}=T_{7}$,

$2 P x \mathcal{T}_{4}=T_{1}-T_{2}-2 T_{7}, \quad 2 P x \mathcal{T}_{5}=-T_{1}+T_{5}+2 T_{8}, \quad 4(P x)^{2} \mathcal{T}_{6}=2 T_{2}-2 T_{3}-2 T_{4}+2 T_{5}+2 T_{7}+2 T_{8}$,

$4 P x \mathcal{T}_{7}=T_{7}-T_{8}, \quad 4(P x)^{2} \mathcal{T}_{8}=-T_{1}+T_{2}+T_{5}-T_{6}+2 T_{7}+2 T_{8}$

In the following we present the calculation of the $O\left(x^{2}\right)$ corrections to the light-cone expansion of the three-quark operator in Eq. C.1 in a simplified situation, where positions of two of the three 
quarks coincide. This approximation is sufficient for the derivation of LCSRs to the tree-level accuracy. The strategy is based on the approach developed in $94,95,96$. We present a slightly more expanded derivation than it was given in [35], where only $\mathcal{V}_{1}^{M}$ was obtained. Note that considering the vector and axial-vector Lorentz projections is suffi- cient since the tensor ones can be determined with the help of isospin relations 83 .

Consider first the case where positions of the two $u$-quarks coincide. This situation occurs when the $d$-quark interacts with the (electromagnetic) probe, hence we refer to it as the $d$-quark contribution:

$$
\begin{array}{r}
x^{\alpha}\left\langle 0\left|\varepsilon^{i j k}\left[u^{i} C \gamma_{\alpha} u^{j}\right](0) d_{\gamma}^{k}(x)\right| P\right\rangle=-x^{\alpha}\left[\left(\mathcal{V}_{1}+\frac{x^{2} m_{N}^{2}}{4} \mathcal{V}_{1}^{M(d)}\right) P_{\alpha}\left(\gamma_{5} N\right)_{\gamma}+\mathcal{V}_{2} m_{N} P_{\alpha}\left(\not x \gamma_{5} N\right)_{\gamma}\right. \\
\left.+\mathcal{V}_{3} m_{N}\left(\gamma_{\alpha} \gamma_{5} N\right)_{\gamma}+\mathcal{V}_{4} m_{N}^{2} x_{\alpha}\left(\gamma_{5} N\right)_{\gamma}+\mathcal{V}_{6} m_{N}^{3} x_{\alpha}\left(\not x \gamma_{5} N\right)_{\gamma}\right], \\
x^{\alpha}\left\langle 0\left|\varepsilon^{i j k}\left[u^{i} C \gamma_{\alpha} \gamma_{5} u^{j}\right](0) d_{\gamma}^{k}(x)\right| P\right\rangle=-x^{\alpha}\left[\left(\mathcal{A}_{1}+\frac{x^{2} m_{N}^{2}}{4} \mathcal{A}_{1}^{M(d)}\right) P_{\alpha}\left(\gamma_{5} N\right)_{\gamma}+\mathcal{A}_{2} m_{N} P_{\alpha}\left(\not x \gamma_{5} N\right)_{\gamma}\right. \\
\left.+\mathcal{A}_{3} m_{N}\left(\gamma_{\alpha} \gamma_{5} N\right)_{\gamma}+\mathcal{A}_{4} m_{N}^{2} x_{\alpha}\left(\gamma_{5} N\right)_{\gamma}+\mathcal{A}_{6} m_{N}^{3} x_{\alpha}\left(\not x \gamma_{5} N\right)_{\gamma}\right] .
\end{array}
$$

We remind that $\mathcal{V}_{1}, \mathcal{A}_{1}$ start at leading twist3 , and hence $\mathcal{V}_{1}^{M(d)}, \mathcal{A}_{1}^{M(d)}$ are of twist-5. Strictly speaking, since we are not taking into account twist- 6 contributions induced by $O\left(x^{2}\right)$ corrections to $\mathcal{V}_{2}, \mathcal{A}_{2}$ and $\mathcal{V}_{3}, \mathcal{A}_{3}$, in order to be consistent we have to discard the contribution of $\mathcal{V}_{6}$ and $\mathcal{A}_{6}$ altogether. This contribution to the sum rules appears to be numerically negligible, however.

For definiteness, consider the vector projection. The meaning of the separation between $\mathcal{V}_{1}$ and $\mathcal{V}_{1}^{M(d)}$ is most easily understood upon the short distance expansion $x_{\mu} \rightarrow 0$. In this way, the nonlo- cal "light-ray" operator in the l.h.s. of Eq. C.3. is Taylor-expanded in a series of local operators with three quark fields and the increasing number of (covariant) derivatives acting on the d-quark. The separation of the leading twist part of each local operator corresponds to the symmetrisation over all Lorentz indices and the subtraction of traces. Without loss of generality, we can consider the matrix element contracted with an additional factor $x_{\alpha}$, see Eq. C.3 , so that the symmetrisation is achieved. To subtract the traces, we formally write

$$
\left.x_{\alpha} d(x)\right|_{\mathrm{lt}}=\sum_{n=0}^{\infty} \frac{1}{n !}\left[x_{\alpha} x_{\mu_{1}} \ldots x_{\mu_{n}}-\frac{x^{2}}{4}\left(\frac{2}{n+1}\right) \sum_{\mu_{i}, \mu_{j}}\left(x_{\alpha} \ldots g_{\mu_{i} \mu_{j}} \ldots x_{\mu_{n}}\right)\right] \partial^{\mu_{1}} \ldots \partial^{\mu_{n}} d(0),
$$

where 'lt' stands for the leading-twist part. Observing that $\frac{1}{n+1}=\int_{0}^{1} \mathrm{~d} t t^{n}$ the subtracted contributions $O\left(x^{2}\right)$ can be reassembled in the form of a non-local string operator:

$$
\begin{aligned}
& \left\langle 0\left|\varepsilon^{i j k}\left[u^{i} C \not x u^{j}\right](0) d_{\gamma}^{k}(x)\right| P\right\rangle= \\
& =\left\langle 0\left|\left[\varepsilon^{i j k}\left[u^{i} C \not x u^{j}\right](0) d_{\gamma}^{k}(x)\right]_{1-\mathrm{t}}\right| P\right\rangle
\end{aligned}
$$

$$
+\frac{x^{2}}{4} \int_{0}^{1} \mathrm{~d} t \frac{\partial^{2}}{\partial x_{\alpha} \partial x^{\alpha}}\left\langle 0\left|\varepsilon^{i j k}\left[u^{i} C \not x u^{j}\right](0) d_{\gamma}^{k}(t x)\right| P\right\rangle
$$

Alternatively, the same result can be obtained by observing 94 that the leading-twist light-ray operator has to satisfy the homogeneous Laplace equa- 
tion

$$
\frac{\partial^{2}}{\partial x_{\lambda} \partial x^{\lambda}}\left\langle 0\left|\left[\varepsilon^{i j k}\left[u^{i} C \not \not u^{j}\right](0) d_{\gamma}^{k}(x)\right]_{\mathrm{lt}}\right| P\right\rangle=0 .
$$

Using QCD equations of motion the third line in Eq. C.5. can be simplified to

$$
\begin{aligned}
& \frac{\partial^{2}}{\partial x_{\alpha} \partial x^{\alpha}} \varepsilon^{i j k}\left[u^{i} C \not x u^{j}\right](0) d_{\gamma}^{k}(t x)= \\
& =2 t \varepsilon^{i j k}\left[u^{i} C \gamma^{\alpha} u^{j}\right](0) D_{\alpha} d_{\gamma}^{k}(t x)+\text { gluons } \\
& =2 t \partial_{\alpha} \varepsilon^{i j k}\left[u^{i} C \gamma^{\alpha} u^{j}\right](0) d_{\gamma}^{k}(t x)+\text { gluons }
\end{aligned}
$$

where $\partial_{\alpha}$ is a derivative with respect to the overall translation 94]; for the matrix element we can make the substitution $\partial_{\alpha} \rightarrow-i P_{\alpha}$. Inserting this result in Eq. C.5 we finally obtain

$$
\begin{aligned}
\left\langle 0\left|\varepsilon^{i j k}\left[u^{i} C \not x u^{j}\right](0) d_{\gamma}^{k}(x)\right| P\right\rangle= \\
=\left\langle 0\left|\left[\varepsilon^{i j k}\left[u^{i} C \not x u^{j}\right](0) d_{\gamma}^{k}(x)\right]_{\mathrm{lt}}\right| P\right\rangle+\frac{x^{2}}{4}\left(-i 2 P_{\alpha}\right) \\
\\
\quad \int_{0}^{1} \mathrm{~d} t t\left\langle 0\left|\varepsilon^{i j k}\left[u^{i} C \gamma^{\alpha} u^{j}\right](0) d_{\gamma}^{k}(t x)\right| P\right\rangle \\
\quad+\text { gluons. }
\end{aligned}
$$

Notice that the r.h.s. only involves (up to corrections with additional gluons) the already known distribution amplitudes. This equation therefore allows us to determine $\mathcal{V}^{M(d)}$ - which appears on the l.h.s. of Eq. C.8 - up to gluonic corrections.

Consider the first term on the r.h.s. of Eq. (C.8). We can write

$$
\begin{aligned}
\langle 0| & {\left[\varepsilon^{i j k}\left[u^{i} C \not x u^{j}\right](0) d_{\gamma}^{k}(x)\right]_{\mathrm{lt}}|P\rangle=} \\
= & -\int \mathcal{D} x\left[e^{-i P \cdot x x_{3}}(P x)\right]_{\mathrm{lt}} \mathcal{V}_{1}\left(\gamma_{5} N\right)_{\gamma} \\
& -\int \mathcal{D} x\left[e^{-i P \cdot x x_{3}}\left(\not x \gamma_{5} N\right)_{\gamma}\right]_{\mathrm{lt}}(P x) \mathcal{V}_{2} m_{N} \\
& -\int \mathcal{D} x\left[e^{-i P \cdot x x_{3}}\left(\not x \gamma_{5} N\right)_{\gamma}\right]_{\mathrm{lt}} \mathcal{V}_{3} m_{N}+\ldots(.
\end{aligned}
$$

where $\left[e^{-i P \cdot x x_{3}}(P \cdot x)\right]_{\mathrm{lt}}$ and $\left[e^{-i P \cdot x x_{3}} \not x\right]_{\mathrm{lt}}$ are the leading-twist components for the free fields, defined as the solutions of the corresponding homogeneous Laplace equation [95]. Note that the factor $P x$ in the second line in Eq. [C.9) is not included under the $[\ldots]_{\text {lt }}$ bracket since $(P x) \mathcal{V}_{2}=1 / 2\left(V_{1}-V_{2}-V_{3}\right)$ is a function of momentum fractions only and does not contain any dependence on the position vector $x$. To the required $\mathcal{O}\left(x^{2}\right)$ accuracy, the leadingtwist exponents $\left[e^{-i P \cdot x x_{3}} \ldots\right]_{1 \mathrm{t}}$ can be obtained from the expression given in 95 ]:

$$
\left[e^{-i P \cdot x x_{3}}\right]_{1 \mathrm{t}}=e^{-i P \cdot x x_{3}}+\frac{x^{2} m_{N}^{2} x_{3}^{2}}{4} \int_{0}^{1} \mathrm{~d} t e^{-i P \cdot x x_{3} t},
$$

by taking the derivative with respect to $x_{3}$ and with respect to $P_{\mu}$. One gets

$$
\begin{gathered}
{\left[e^{-i P \cdot x x_{3}}(P \cdot x)\right]_{\mathrm{lt}}=(P x)\left[e^{-i P \cdot x x_{3}}\right.} \\
\left.+\frac{x^{2} m_{N}^{2} x_{3}^{2}}{4} \int_{0}^{1} \mathrm{~d} t e^{-i P \cdot x x_{3} t}\right], \\
{\left[e^{-i P \cdot x_{3}} \not x\right]_{\mathrm{lt}}=\not x\left[e^{-i P \cdot x x_{3}}\right.} \\
\left.+\frac{1}{4} x^{2} m_{N}^{2} x_{3}^{2} \int_{0}^{1} \mathrm{~d} t t^{2} e^{-i P \cdot x x_{3} t}\right] \\
+\frac{i}{2} \not P x_{3} x^{2} \int_{0}^{1} \mathrm{~d} t t e^{-i P \cdot x x_{3} t} .
\end{gathered}
$$

Note that we have corrected a misprint in 35] in the last term of the second equation, where a factor $1 / 2$ arises instead of $1 / 4$.

The corresponding contribution to $\mathcal{V}_{1}^{M(d)}$ is proportional to the nucleon mass squared and involves the leading twist distribution amplitude, being an exact analogue of the Nachtmann power suppressed correction in deep inelastic scattering. The second contribution on the r.h.s. in Eq. (C.8) is special for the exclusive kinematics since it involves a derivative over the total translation that vanishes for forward matrix elements. Its explicit form is easily found by contracting the three-quark matrix element in Eq. C.3 with $P_{\alpha}$ instead of $x_{\alpha}$ and inserting the resulting expression in Eq. [C.8). One gets

$$
\begin{aligned}
& \frac{x^{2}}{4}\left(-2 i P_{\alpha}\right) \int_{0}^{1} \mathrm{~d} t t\left\langle 0\left|\varepsilon^{i j k}\left[u^{i} C \gamma^{\alpha} u^{j}\right](0) d_{\gamma}^{k}(t x)\right| P\right\rangle= \\
& =\frac{x^{2} m_{N}^{2}}{4} i \int \mathcal{D} x \int_{0}^{1} \mathrm{~d} t t e^{-i P \cdot x x_{3} t}\left(V_{1}+V_{5}\right)\left(\gamma_{5} N\right)_{\gamma} \\
& \quad+\ldots,
\end{aligned}
$$

where the ellipses stand for other Lorentz structures that do not contribute to $\mathcal{V}_{1}^{M(d)}$. Inserting everything into Eq. C.5 we arrive at

$$
\begin{aligned}
& (P x) \int \mathrm{d} x_{3} e^{-i x_{3} P \cdot x} \mathcal{V}_{1}^{M(d)}\left(x_{3}\right)= \\
& =(P x) \int \mathcal{D} x x_{3}^{2} \int_{0}^{1} \mathrm{~d} t e^{-i P \cdot x x_{3} t} V_{1} \\
& \quad-i \int \mathcal{D} x x_{3} \int_{0}^{1} \mathrm{~d} t t e^{-i t x_{3} P \cdot x}\left(V_{1}-V_{2}\right) \\
& +\frac{1}{P x} \int \mathcal{D} x e^{-i x_{3} P \cdot x}\left(-2 V_{1}+V_{3}+V_{4}+2 V_{5}\right) .
\end{aligned}
$$


In order to solve this equation we expand both sides at short distances and obtain the moments of $\mathcal{V}_{1}^{M(d)}$ with respect to $x_{3}$ expressed through moments of the distribution amplitudes defined as $V_{i}^{(d)(n)}=$ $\int \mathcal{D} x x_{3}^{n} V_{i}\left(x_{i}\right)$. One finds

$$
\begin{aligned}
& \int \mathrm{d} x_{3} x_{3}^{n} \mathcal{V}_{1}^{M(d)}\left(x_{3}\right)=\frac{1}{n+1}\left[V_{1}^{(d)(n+2)}\right. \\
& -\frac{1}{n+3}\left(V_{1}-V_{2}\right)^{(d)(n+2)}-\frac{1}{n+3}\left(V_{1}+V_{5}\right)^{(d)(n+1)} \\
& \left.+\frac{1}{n+2}\left(-2 V_{1}+V_{3}+V_{4}+2 V_{5}\right)^{(d)(n+2)}\right], \quad(\text { C. } 14)
\end{aligned}
$$

up to contributions of multiparton distribution amplitudes with extra gluons that have been neglected. The corresponding expression for $\mathcal{V}_{1}^{M(d)}\left(x_{3}\right)$ in the momentum fraction space is easily obtained by inserting the conformal expansions for $V_{1}, \ldots, V_{6}$ and inverting the moment equation, see below.

The analysis of the $u$-quark contribution is performed in a similar way. We consider the matrix element

$$
\begin{array}{r}
x^{\alpha}\left\langle 0\left|\varepsilon^{i j k}\left[u^{i}(0) C \gamma_{\alpha} u^{j}(x)\right] d_{\gamma}^{k}(0)\right| P\right\rangle=-x^{\alpha}\left[\left(\mathcal{V}_{1}+\frac{x^{2} m_{N}^{2}}{4} \mathcal{V}_{1}^{M(u)}\right) P_{\alpha}\left(\gamma_{5} N\right)_{\gamma}+\mathcal{V}_{2} m_{N} P_{\alpha}\left(\not x \gamma_{5} N\right)_{\gamma}\right. \\
\left.+\mathcal{V}_{3} m_{N}\left(\gamma_{\alpha} \gamma_{5} N\right)_{\gamma}+\mathcal{V}_{4} m_{N}^{2} x_{\alpha}\left(\gamma_{5} N\right)_{\gamma}+\mathcal{V}_{6} m_{N}^{3} x_{\alpha}\left(\not x \gamma_{5} N\right)_{\gamma}\right]
\end{array}
$$

and find repeating the same steps that lead to Eq. (C.5):

$$
\begin{aligned}
& \left\langle 0\left|\varepsilon^{i j k}\left[u^{i}(0) C \not x u^{j}(x)\right] d_{\gamma}^{k}(0)\right| P\right\rangle=\left\langle 0\left|\left[\varepsilon^{i j k}\left[u^{i}(0) C \not x u^{j}(x)\right] d_{\gamma}^{k}(0)\right]_{\mathrm{lt}}\right| P\right\rangle \\
& +\frac{x^{2}}{4} \int_{0}^{1} \mathrm{~d} t \frac{\partial^{2}}{\partial x_{\alpha} \partial x^{\alpha}}\left\langle 0\left|\varepsilon^{i j k}\left[u^{i}(0) C \not x u^{j}(t x)\right] d_{\gamma}^{k}(0)\right| P\right\rangle=\left\langle 0\left|\left[\varepsilon^{i j k}\left[u^{i}(0) C \not x u^{j}(x)\right] d_{\gamma}^{k}(0)\right]_{\mathrm{lt}}\right| P\right\rangle+\text { gluons, }
\end{aligned}
$$

the only difference being that the term corresponding to a total translation does not arise in this case. For the moments with respect to $x_{2}$ we get

$$
\begin{aligned}
& \int \mathrm{d} x_{2} x_{2}^{n} \mathcal{V}_{1}^{M(u)}\left(x_{2}\right)=\frac{1}{n+1}\left[V_{1}^{(u)(n+2)}\right. \\
& -\frac{1}{n+3}\left(V_{1}-V_{2}\right)^{(u)(n+2)} \\
& \left.+\frac{1}{n+2}\left(-2 V_{1}+V_{3}+V_{4}+2 V_{5}\right)^{(u)(n+2)}\right] .
\end{aligned}
$$

Inserting the conformal expansions for $V_{1}, \ldots, V_{6}$ and inverting the moment equations we find

$$
\begin{aligned}
\mathcal{V}_{1}^{M(u)}\left(x_{2}\right) & =\int_{0}^{1-x_{2}} d x_{1} V_{1}^{M}\left(x_{1}, x_{2}, 1-x_{1}-x_{2}\right) \\
& =\frac{x_{2}^{2}}{24}\left(f_{N} C_{f}^{u}+\lambda_{1} C_{\lambda}^{u}\right),
\end{aligned}
$$

$$
\begin{aligned}
\mathcal{V}_{1}^{M(d)}\left(x_{3}\right) & =\int_{0}^{1-x_{3}} d x_{1} V_{1}^{M}\left(x_{1}, 1-x_{1}-x_{3}, x_{3}\right) \\
& =\frac{x_{3}^{2}}{24}\left(f_{N} C_{f}^{d}+\lambda_{1} C_{\lambda}^{d}\right)
\end{aligned}
$$

with

$$
\begin{aligned}
C_{f}^{u}= & \left(1-x_{2}\right)^{3}\left[113+495 x_{2}-552 x_{2}^{2}\right. \\
& -10 A_{1}^{u}\left(1-3 x_{2}\right) \\
& \left.+2 V_{1}^{d}\left(113-951 x_{2}+828 x_{2}^{2}\right)\right], \\
C_{\lambda}^{u}= & -\left(1-x_{2}\right)^{3}\left[13-20 f_{1}^{d}+3 x_{2}+10 f_{1}^{u}\left(1-3 x_{2}\right)\right], \\
C_{f}^{d}= & -\left(1-x_{3}\right)\left[1441+505 x_{3}-3371 x_{3}^{2}+3405 x_{3}^{3}\right. \\
& -1104 x_{3}^{4}-24 V_{1}^{d}\left(207-3 x_{3}-368 x_{3}^{2}+412 x_{3}^{3}\right. \\
& \left.\left.-138 x_{3}^{4}\right)\right]-12\left(73-220 V_{1}^{d}\right) \ln \left[x_{3}\right], \\
C_{\lambda}^{d}= & -\left(1-x_{3}\right)\left[11+131 x_{3}-169 x_{3}^{2}+63 x_{3}^{3}\right. \\
& \left.-30 f_{1}^{d}\left(3+11 x_{3}-17 x_{3}^{2}+7 x_{3}^{3}\right)\right]
\end{aligned}
$$




$$
-12\left(3-10 f_{1}^{d}\right) \ln \left[x_{3}\right]
$$

This result agrees with [35].

Similarly we get for the axial-vector functions:

$$
\begin{aligned}
& \int \mathrm{d} x_{3} x_{3}^{n} \mathcal{A}_{1}^{M(d)}\left(x_{3}\right)=\frac{1}{n+1}\left[A_{1}^{(d)(n+2)}\right. \\
& -\frac{1}{n+3}\left(A_{1}-A_{2}\right)^{(d)(n+2)} \\
& +\frac{1}{n+2}\left(-2 A_{1}-A_{3}-A_{4}+2 A_{5}\right)^{(d)(n+2)} \\
& \left.-\frac{1}{n+3}\left(A_{1}+A_{5}\right)^{(d)(n+1)}\right], \\
& \int \mathrm{d} x_{2} x_{2}^{n} \mathcal{A}_{1}^{M(u)}\left(x_{2}\right)=\frac{1}{n+1}\left[A_{1}^{(u)(n+2)}\right. \\
& -\frac{1}{n+3}\left(A_{1}-A_{2}\right)^{(u)(n+2)} \\
& \left.+\frac{1}{n+2}\left(-2 A_{1}-A_{3}-A_{4}+2 A_{5}\right)^{(u)(n+2)}\right] .
\end{aligned}
$$

which is solved by

$$
\begin{aligned}
\mathcal{A}_{1}^{M(u)}\left(x_{2}\right) & =\int_{0}^{1-x_{2}} d x_{1} A_{1}^{M}\left(x_{1}, x_{2}, 1-x_{1}-x_{2}\right) \\
& =\frac{x_{2}^{2}}{24}\left(1-x_{2}\right)^{3}\left(f_{N} D_{f}^{u}+\lambda_{1} D_{\lambda}^{u}\right), \\
\mathcal{A}_{1}^{M(d)}\left(x_{3}\right) & =\int_{0}^{1-x_{3}} d x_{1} A_{1}^{M}\left(x_{1}, 1-x_{1}-x_{3}, x_{3}\right) \\
& =0,
\end{aligned}
$$

with

$$
\begin{aligned}
D_{f}^{u}= & 11+45 x_{2}-2 A_{1}^{u}\left(113-951 x_{2}+828 x_{2}^{2}\right) \\
& +10 V_{1}^{d}\left(1-30 x_{2}\right), \\
D_{\lambda}^{u}= & 29-45 x_{2}-10 f_{1}^{u}\left(7-9 x_{2}\right)-20 f_{1}^{d}\left(5-6 x_{2}\right) .
\end{aligned}
$$

Finally, using the isospin relation in Eq. (2.20) of [83] one obtains the $T_{1}^{M}$ functions in terms of $V_{1}^{M}$ and $A_{1}^{M}$ :

$\mathcal{T}_{1}^{M(u)}(x)=\frac{1}{2}\left[V_{1}^{M(d)}(x)+V_{1}^{M(u)}(x)-A_{1}^{M(u)}(x)\right]$,

$\mathcal{T}_{1}^{M(d)}(x)=V_{1}^{M(u)}(x)+A_{1}^{M(u)}(x)$.

Inserting the above expressions we get:

$$
\begin{aligned}
& \mathcal{T}_{1}^{M(u)}(x)=\frac{x^{2}}{48}\left(f_{N} E_{f}^{u}+\lambda_{1} E_{\lambda}^{u}\right), \\
& \mathcal{T}_{1}^{M(d)}(x)=\frac{x^{2}(1-x)^{3}}{6}\left(f_{N} E_{f}^{d}+\lambda_{1} E_{\lambda}^{d}\right)(\mathrm{C} .24)
\end{aligned}
$$

with

$$
\begin{aligned}
E_{f}^{u}= & -\left[( 1 - x ) \left(1339+259 x-2021 x^{2}+1851 x^{3}\right.\right. \\
& -552 x^{4}-72 A_{1}^{u}(1-x)^{3}(3-23 x) \\
& \left.\left.-24 V_{1}^{d}\left(216-99 x-134 x^{2}+196 x^{3}-69 x^{4}\right)\right)\right] \\
& -12\left(73-220 V_{1}^{d}\right) \ln [x], \\
E_{\lambda}^{u}= & -\left[( 1 - x ) \left(53+5 x-43 x^{2}+21 x^{3}\right.\right. \\
& \left.\left.-30\left(7-x-5 x^{2}+3 x^{3}\right) f_{1}^{d}-60(1-x)^{3} f_{1}^{u}\right)\right] \\
& -12\left(3-10 f_{1}^{d}\right) \ln [x], \\
E_{f}^{d=} & 31+135 x-138 x^{2} \\
& -\left(59-483 x+414 x^{2}\right)\left(A_{1}^{u}-V_{1}^{d}\right), \\
E_{\lambda}^{d=} & 4(1-3 x)-10(2-3 x)\left(f_{1}^{d}+f_{1}^{u}\right) .
\end{aligned}
$$

Note that the $x^{2}$-corrections do not depend on $\lambda_{2}$. Our results agree with the ones obtained in [45].

\section{APPENDIX D: ASYMPTOTIC DISTRIBUTION AMPLITUDES}

For completeness we present the set of DAs that is obtained by setting contributions of higher conformal spin operators to zero, cf. (B.14). The subtlety is that conformal symmetry is broken by nucleon mass corrections. As a consequence, "kinematic" higher twist corrections of higher spin have to be retained in order to satisfy EOM. For the relevant parameters we get: for twist-3

$$
\phi_{3}^{0}=f_{N}, \quad \phi_{3}^{-}=0, \quad \phi_{3}^{+}=0 ;
$$

for twist-4

$\phi_{4}^{0}=\frac{1}{2}\left(f_{N}+\lambda_{1}\right), \quad \phi_{4}^{+}=-\frac{1}{12} f_{N}, \quad \phi_{4}^{-}=-\frac{5}{4} f_{N}$,

$\psi_{4}^{0}=\frac{1}{2}\left(f_{N}-\lambda_{1}\right), \quad \psi_{4}^{+}=-\frac{1}{12} f_{N}, \quad \psi_{4}^{-}=\frac{5}{4} f_{N}$,

$\xi_{4}^{0}=\frac{1}{6} \lambda_{2}, \quad \xi_{4}^{+}=0, \quad \xi_{4}^{-}=0$

for twist-5

$$
\begin{aligned}
& \phi_{5}^{0}=\frac{1}{2}\left(f_{N}+\lambda_{1}\right), \quad \psi_{5}^{0}=\frac{1}{2}\left(f_{N}-\lambda_{1}\right) \\
& \phi_{5}^{+}=-\frac{1}{18}\left(65 f_{N}+3 \lambda_{1}\right), \quad \phi_{5}^{-}=-\frac{1}{3}\left(5 f_{N}-\lambda_{1}\right), \\
& \psi_{5}^{+}=-\frac{1}{18}\left(65 f_{N}-3 \lambda_{1}\right), \quad \psi_{5}^{-}=\frac{1}{3}\left(5 f_{N}+\lambda_{1}\right), \\
& \xi_{5}^{0}=\frac{1}{6} \lambda_{2}, \quad \xi_{5}^{+}=-\frac{1}{18} \lambda_{2}, \quad \xi_{5}^{-}=-\frac{1}{3} \lambda_{2}, \quad \text { (D.3) }
\end{aligned}
$$


and for twist- 6

$$
\begin{aligned}
& \phi_{6}^{0}=f_{N}, \quad \phi_{6}^{+}=-\frac{1}{30}\left(5 f_{N}+6 \lambda_{1}\right), \\
& \phi_{6}^{-}=\frac{1}{10}\left(5 f_{N}-2 \lambda_{1}\right) .
\end{aligned}
$$

The corresponding twist-3 DAs are:

$$
\begin{aligned}
& V_{1}\left(x_{i}\right)=120 x_{1} x_{2} x_{3} f_{N}, \quad A_{1}\left(x_{i}\right)=0 \\
& T_{1}\left(x_{i}\right)=120 x_{1} x_{2} x_{3} f_{N}
\end{aligned}
$$

twist-4:

$$
\begin{aligned}
& V_{2}\left(x_{i}\right)=2 x_{1} x_{2}\left[5\left(1+x_{3}\right) f_{N}+6 \lambda_{1}\right], \\
& A_{2}\left(x_{i}\right)=30 x_{1} x_{2}\left(x_{1}-x_{2}\right) f_{N} \\
& T_{2}\left(x_{i}\right)=4 x_{1} x_{2} \lambda_{2} \\
& V_{3}\left(x_{i}\right)=x_{3}\left[5 \left(1+2 x_{1} x_{2}-4 x_{3}\right.\right. \\
& \left.\left.\quad+3\left(x_{1}^{2}+x_{2}^{2}+x_{3}^{2}\right)\right) f_{N}-6\left(1-x_{3}\right) \lambda_{1}\right] \\
& A_{3}\left(x_{i}\right)=-2\left(x_{1}-x_{2}\right) x_{3}\left[5\left(2-3 x_{3}\right) f_{N}-3 \lambda_{1}\right], \\
& T_{3}\left(x_{i}\right)=x_{3}\left[5 \left(1+2 x_{1} x_{2}+2 x_{3}\right.\right. \\
& \left.\left.\quad-3\left(x_{1}^{2}+x_{2}^{2}+x_{3}^{2}\right)\right) f_{N}+\left(1-x_{3}\right) \lambda_{2}\right], \\
& T_{7}\left(x_{i}\right)=x_{3}\left[5 \left(1+2 x_{1} x_{2}+2 x_{3}\right.\right. \\
& \left.\left.\quad-3\left(x_{1}^{2}+x_{2}^{2}+x_{3}^{2}\right)\right) f_{N}-\left(1-x_{3}\right) \lambda_{2}\right]
\end{aligned}
$$

twist-5:

$$
\begin{aligned}
& V_{4}\left(x_{i}\right)=-\frac{1}{3}\left(28-65\left(x_{1}^{2}+x_{2}^{2}\right)-30 x_{1} x_{2}-13 x_{3}\right. \\
& \left.-15 x_{3}^{2}\right) f_{N}-\left(1+\left(x_{1}-x_{2}\right)^{2}-x_{3}^{2}\right) \lambda_{1}, \\
& A_{4}\left(x_{i}\right)=\frac{1}{3}\left(x_{1}-x_{2}\right)\left[\left(37-80 x_{3}\right) f_{N}-6 \lambda_{1}\right], \\
& T_{4}\left(x_{i}\right)=-\frac{1}{6}\left[2 \left(28-65\left(x_{1}^{2}+x_{2}^{2}\right)+30 x_{1} x_{2}-43 x_{3}\right.\right. \\
& \left.\left.+15 x_{3}^{2}\right) f_{N}-\left(1+x_{1}^{2}-6 x_{1} x_{2}+x_{2}^{2}+2 x_{3}-3 x_{3}^{2}\right) \lambda_{2}\right], \\
& T_{8}\left(x_{i}\right)=-\frac{1}{6}\left[2 \left(28-65\left(x_{1}^{2}+x_{2}^{2}\right)+30 x_{1} x_{2}-43 x_{3}\right.\right. \\
& \left.\left.+15 x_{3}^{2}\right) f_{N}+\left(1+x_{1}^{2}-6 x_{1} x_{2}+x_{2}^{2}+2 x_{3}-3 x_{3}^{2}\right) \lambda_{2}\right], \\
& V_{5}\left(x_{i}\right)=x_{3}\left[-\frac{2}{3}\left(28-65 x_{3}\right) f_{N}+2\left(1+x_{3}\right) \lambda_{1}\right], \\
& A_{5}\left(x_{i}\right)=2\left(x_{1}-x_{2}\right) x_{3}\left(5 f_{N}-\lambda_{1}\right),
\end{aligned}
$$

$T_{5}\left(x_{i}\right)=\frac{2}{3} x_{3}\left(1+x_{3}\right) \lambda_{2}$.

and twist-6:

$$
\begin{aligned}
& V_{6}\left(x_{i}\right)=\frac{1}{3}\left(5+3 x_{3}\right) f_{N}-\frac{2}{5}\left(1-3 x_{3}\right) \lambda_{1}, \\
& A_{6}\left(x_{i}\right)=-\frac{1}{5}\left(x_{1}-x_{2}\right)\left(5 f_{N}-2 \lambda_{1}\right), \\
& T_{6}\left(x_{i}\right)=\frac{1}{3}\left(8-6 x_{3}\right) f_{N} .
\end{aligned}
$$

The corresponding expressions for the $x^{2}$ corrections read:

$$
\begin{aligned}
& \mathcal{V}_{1}^{M(d)}(x)=\frac{x^{2}}{24}\left[( 1 - x ) \left(\left(215-529 x+427 x^{2}\right.\right.\right. \\
& \left.\left.\left.-109 x^{3}\right)+4 \ln [x]\right) f_{N}+16(1-x)^{3} \lambda_{1}\right], \\
& \mathcal{V}_{1}^{M(u)}(x)=\frac{x^{2}}{72}(1-x)^{3}\left[(565-417 x) f_{N}-24 \lambda_{1}\right], \\
& \mathcal{A}_{1}^{M(u)}(x)=\frac{x^{2}}{72}(1-x)^{3}\left[(43+105 x) f_{N}-24 \lambda_{1}\right], \\
& \mathcal{T}_{1}^{M(d)}(x)=\frac{x^{2}}{9}(1-x)^{3}\left[(76-39 x) f_{N}-6 \lambda_{1}\right], \\
& \mathcal{T}_{1}^{M(u)}(x)=\frac{x^{2}}{48}\left[\left(( 1 - x ) \left(389-1051 x+949 x^{2}\right.\right.\right. \\
& \left.\left.\left.-283 x^{3}\right)+4 \ln [x]\right) f_{N}+16(1-x)^{3} \lambda_{1}\right] .
\end{aligned}
$$

\section{APPENDIX E: QCD SUM RULES}

In this Appendix we update the QCD sum rules for the shape parameters of the higher-twist DAs, which are defined as 35,83

$$
\begin{array}{r}
\left\langle 0\left|\left(u(0) C \gamma_{\mu} u(0)\right) \not k \gamma_{5} \gamma^{\mu}(i z \vec{D} d)(0)\right| P\right\rangle= \\
=\lambda_{1} f_{1}^{d}(p z) M \not N(P), \\
\begin{aligned}
\left\langle 0\left|\left(u^{a}(0) C \sigma_{\mu \nu} u(0)\right) \not \gamma_{5} \sigma^{\mu \nu}(i z \vec{D} d)(0)\right| P\right\rangle= \\
=\lambda_{2} f_{2}^{d}(p z) M \not N(P),
\end{aligned} \\
\begin{aligned}
\langle 0|\left(u(0) C \gamma_{\mu} \gamma_{5} i z \stackrel{\leftrightarrow}{D} u(0)\right) & \not \gamma^{\mu} d(0)|P\rangle= \\
& =\lambda_{1} f_{1}^{u}(p z) M \not N(P),
\end{aligned}
\end{array}
$$

where we have used the notation $i z \cdot \stackrel{\leftrightarrow}{D}=i z \cdot(\vec{D}-$ $\overleftarrow{D})$ and for brevity omitted color indices.

The QCD sum rule estimates for $f_{1}^{d}, f_{2}^{d}, f_{1}^{u}$ are derived from the consideration of the two-point correlation functions 


$$
\begin{aligned}
& i \int \mathrm{d}^{4} x e^{i p x}\left\langle 0\left|T\left\{\varepsilon^{i j k}\left(u^{i}(x) C \gamma_{\mu} u^{j}(x)\right) \gamma_{5} \gamma^{\mu}(i z \vec{D} d)^{k}(x) \bar{\eta}_{1}(0)\right\}\right| 0\right\rangle=\frac{f_{1}^{d}\left|\lambda_{1}\right|^{2} M^{2} p \cdot z(\not p+M)}{M^{2}-p^{2}}+\ldots, \\
& i \int \mathrm{d}^{4} x e^{i p x}\left\langle 0\left|T\left\{\varepsilon^{i j k}\left(u(x) C \gamma_{\mu} \gamma_{5} i z \stackrel{\leftrightarrow}{D} u(x)\right)^{i j} \gamma^{\mu} d^{k}(x) \bar{\eta}_{1}(0)\right\}\right| 0\right\rangle=\frac{f_{1}^{u}\left|\lambda_{1}\right|^{2} M^{2} p \cdot z(\not p+M)}{M^{2}-p^{2}}+\ldots \\
& i \int \mathrm{d}^{4} x e^{i p x}\left\langle 0\left|T\left\{\varepsilon^{i j k}\left(u^{i}(x) C \sigma_{\mu \nu} u^{j}(x)\right) \gamma_{5} \sigma^{\mu \nu}(i z \vec{D} d)^{k}(x) \bar{\eta}_{2}(0)\right\}\right| 0\right\rangle=\frac{f_{2}^{d}\left|\lambda_{2}\right|^{2} M^{2} p \cdot z(\not p+M)}{M^{2}-p^{2}}+\ldots
\end{aligned}
$$

The dots refer to contributions of excited states and different Lorentz structures that we do not consider. We have calculated the correlation functions in (E.2) using a more consistent factorization approximation for the contribution of dimension- 8 operators compared to [83], which takes into account the contribution of nonplanar diagrams. For example, we use

$$
\begin{aligned}
\epsilon^{i j k} \epsilon^{i^{\prime} j^{\prime} k^{\prime}}\left\langle 0\left|\left(u^{i} C \gamma_{\mu}\left[\vec{D}_{\alpha} \vec{D}_{\beta}+\vec{D}_{\beta} \vec{D}_{\alpha}\right]^{k l} u^{l}\right)\left(\bar{u}^{i^{\prime}} \gamma_{\nu} C \bar{u}^{k^{\prime}}\right)\right| 0\right\rangle & =\frac{m_{0}^{2}\langle\bar{u} u\rangle^{2}}{216}\left[19 g_{\mu \nu} g_{\alpha \beta}-2\left(g_{\mu \alpha} g_{\nu \beta}-g_{\nu \alpha} g_{\mu \beta}\right)\right], \\
\epsilon^{i j k} \epsilon^{i^{\prime} j^{\prime} k^{\prime}}\left\langle 0\left|\left(u^{i} C \sigma_{\mu \nu}\left[\vec{D}_{\xi} \vec{D}_{\eta}+\vec{D}_{\eta} \vec{D}_{\xi}\right]^{k l} u^{l}\right)\left(\bar{u}^{i^{\prime}} \sigma_{\alpha \beta} C \bar{u}^{k^{\prime}}\right)\right| 0\right\rangle & =\frac{5 m_{0}^{2}\langle\bar{u} u\rangle^{2}}{72} g_{\xi \eta}\left(g_{\mu \alpha} g_{\nu \beta}-g_{\nu \alpha} g_{\mu \beta}\right), \quad \text { (E.3) }
\end{aligned}
$$

where $\langle\bar{u} u\rangle$ is the $u$-quark condensate and $m_{0}^{2}=\langle\bar{u} \sigma g G u\rangle /\langle\bar{u} u\rangle$. Following the standard procedure and replacing $\left|\lambda_{1}\right|^{2}$ and $\left|\lambda_{2}\right|^{2}$ by the corresponding sum rules derived from the diagonal correlation functions of the $\eta_{1}$ and $\eta_{2}$ currents, respectively:

$$
\begin{aligned}
& 2(2 \pi)^{4} m_{N}^{2}\left|\lambda_{1}\right|^{2}=\exp \left(m_{N}^{2} / M^{2}\right)\left\{M^{6} E_{3}\left(s_{0} / M^{2}\right)+\frac{b}{4} M^{2} E_{1}\left(s_{0} / M^{2}\right)+\frac{a^{2}}{3}\left(4-\frac{4}{3} \frac{m_{0}^{2}}{M^{2}}\right)\right\}, \\
& 2(2 \pi)^{4} m_{N}^{2} \frac{\left|\lambda_{2}\right|^{2}}{6}=\exp \left(m_{N}^{2} / M^{2}\right)\left\{M^{6} E_{3}\left(s_{0} / M^{2}\right)+\frac{b}{4} M^{2} E_{1}\left(s_{0} / M^{2}\right)\right\},
\end{aligned}
$$

we obtain

$$
\begin{aligned}
& f_{1}^{d}=\frac{\frac{3}{10} M^{6} E_{3}\left(s_{0} / M^{2}\right)+\frac{b}{24} M^{2} E_{1}\left(s_{0} / M^{2}\right)+\frac{a^{2}}{3}\left(4-\frac{31}{9} \frac{m_{0}^{2}}{M^{2}}\right)}{M^{6} E_{3}\left(s_{0} / M^{2}\right)+\frac{b}{4} M^{2} E_{1}\left(s_{0} / M^{2}\right)+\frac{a^{2}}{3}\left(4-\frac{4}{3} \frac{m_{0}^{2}}{M^{2}}\right)}, \\
& f_{1}^{u}=\frac{\frac{1}{10} M^{6} E_{3}\left(s_{0} / M^{2}\right)+\frac{b}{8} M^{2} E_{1}\left(s_{0} / M^{2}\right)-\frac{a^{2}}{3} \frac{m_{0}^{2}}{M^{2}}}{M^{6} E_{3}\left(s_{0} / M^{2}\right)+\frac{b}{4} M^{2} E_{1}\left(s_{0} / M^{2}\right)+\frac{a^{2}}{3}\left(4-\frac{4}{3} \frac{m_{0}^{2}}{M^{2}}\right)}, \\
& f_{2}^{d}=\frac{\frac{8}{5} M^{6} E_{3}\left(s_{0} / M^{2}\right)}{6 M^{6} E_{3}\left(s_{0} / M^{2}\right)+\frac{3 b}{2} M^{2} E_{1}\left(s_{0} / M^{2}\right)} .
\end{aligned}
$$

where

$$
E_{n}\left(s_{0} / M^{2}\right)=1-e^{\left(-s_{0} / M^{2}\right)} \sum_{k=0}^{n-1} \frac{1}{k !}\left(\frac{s_{0}}{M^{2}}\right)^{k} .
$$

In all sum rules $M$ is the Borel parameter; we use the interval $1 \mathrm{GeV}^{2} \leq M^{2} \leq 2 \mathrm{GeV}^{2}$, with the continuum threshold $\sqrt{s_{0}} \sim 1.5 \mathrm{GeV}$ and also values of the condensates at the scale $\mu^{2}=1 \mathrm{GeV}^{2}$

$$
a=-(2 \pi)^{2}\langle\bar{q} q\rangle \simeq 0.55 \mathrm{GeV}^{3},
$$

$$
\begin{array}{r}
b=(2 \pi)^{2}\left\langle\frac{\alpha_{S}}{\pi} G^{2}\right\rangle \simeq 0.47 \mathrm{GeV}^{4}, \\
m_{0}^{2}=\frac{\langle\bar{q} g G q\rangle}{\langle\bar{q} q\rangle} \simeq 0.65 \mathrm{GeV}^{2} .
\end{array}
$$

With these inputs we find the numbers quoted in (B.16). These results have smaller errors and supersede the corresponding estimates in Ref. [83]. 
[1] R. W. Mcallister and R. Hofstadter, Phys. Rev. 102, 851 (1956).

[2] S. J. Brodsky and G. R. Farrar, Phys. Rev. Lett. 31, 1153 (1973).

[3] V. A. Matveev, R. M. Muradian and A. N. Tavkhelidze, Lett. Nuovo Cim. 7, 719 (1973).

[4] V. L. Chernyak and A. R. Zhitnitsky, JETP Lett. 25, 510 (1977) Sov. J. Nucl. Phys. 31, 544 (1980); V. L. Chernyak, A. R. Zhitnitsky and V. G. Serbo, JETP Lett. 26, 594 (1977) Sov. J. Nucl. Phys. 31, 552 (1980).

[5] A. V. Radyushkin, JINR report R2-10717 (1977), arXiv:hep-ph/0410276 (English translation); A. V. Efremov and A. V. Radyushkin, Theor. Math. Phys. 42, 97 (1980) Phys. Lett. B 94, 245 (1980).

[6] G. P. Lepage and S. J. Brodsky, Phys. Lett. B 87, 359 (1979); Phys. Rev. D 22, 2157 (1980).

[7] A. Duncan and A. H. Mueller, Phys. Rev. D 21 (1980) 1636.

[8] A. Duncan and A. H. Mueller, Phys. Lett. B 90 (1980) 159.

[9] A. I. Milshtein and V. S. Fadin, Yad. Fiz. 33 (1981) 1391.

[10] A. I. Milshtein and V. S. Fadin, Yad. Fiz. 35 (1982) 1603.

[11] N. Isgur and C. H. Llewellyn Smith, Phys. Rev. Lett. 52, 1080 (1984).

[12] N. Isgur and C. H. Llewellyn Smith, Nucl. Phys. B 317, 526 (1989), Phys. Lett. B 217 (1989) 535.

[13] P. Kroll, M. Schurmann and P. A. M. Guichon, Nucl. Phys. A 598, 435 (1996).

[14] A. V. Radyushkin, Phys. Rev. D 58, 114008 (1998).

[15] M. Diehl, T. Feldmann, R. Jakob and P. Kroll, Eur. Phys. J. C 8, 409 (1999).

[16] K. Goeke, M. V. Polyakov and M. Vanderhaeghen, Prog. Part. Nucl. Phys. 47, 401 (2001).

[17] M. Diehl, Phys. Rept. 388, 41 (2003).

[18] A. V. Belitsky and A. V. Radyushkin, Phys. Rept. 418, 1 (2005).

[19] A. V. Belitsky, X. d. Ji and F. Yuan, Phys. Rev. D 69, 074014 (2004).

[20] M. Diehl, T. Feldmann, R. Jakob and P. Kroll, Eur. Phys. J. C 39, 1 (2005).

[21] M. Guidal, M. V. Polyakov, A. V. Radyushkin and M. Vanderhaeghen, Phys. Rev. D 72, 054013 (2005).

[22] B. L. Ioffe and A. V. Smilga, Nucl. Phys. B 216, 373 (1983).

[23] V. A. Nesterenko and A. V. Radyushkin, Phys. Lett. B 115, 410 (1982).

[24] M. A. Shifman, A. I. Vainshtein and V. I. Zakharov, Nucl. Phys. B 147, 385, 448 (1979).

[25] V. M. Belyaev and I. I. Kogan, Int. J. Mod. Phys. A 8, 153 (1993).

[26] H. Castillo, C. A. Dominguez and M. Loewe, JHEP 0503, 012 (2005).
[27] A. P. Bakulev and A. V. Radyushkin, Phys. Lett. B 271, 223 (1991).

[28] V. A. Nesterenko and A. V. Radyushkin, Phys. Lett. B 128, 439 (1983).

[29] P. Mergell, U. G. Meissner and D. Drechsel, Nucl. Phys. A 596, 367 (1996);

H. W. Hammer, U. G. Meissner and D. Drechsel, Phys. Lett. B 385, 343 (1996);

H. W. Hammer and U. G. Meissner, Eur. Phys. J. A 20, 469 (2004);

M. A. Belushkin, H. W. Hammer and U. G. Meissner, Phys. Lett. B 633, 507 (2006).

[30] R. Baldini, M. Mirazita, S. Pacetti, C. Bini, P. Gauzzi and M. Negrini, Nucl. Phys. A 755, 286 (2005).

[31] M. Göckeler, T. R. Hemmert, R. Horsley, D. Pleiter, P. E. L. Rakow, A. Schäfer and G. Schierholz [QCDSF Collaboration], Phys. Rev. D 71 (2005) 034508.

[32] C. Alexandrou, P. de Forcrand, H. Neff, J. W. Negele, W. Schroers and A. Tsapalis, Phys. Rev. Lett. 94 (2005) 021601.

[33] M. Göckeler et al. [QCDSF Collaboration], Nucl. Phys. Proc. Suppl. 140 (2005) 399.

[34] M. Göckeler et al. [QCDSF Collaboration], Few Body Syst. 36 (2005) 111.

[35] V. M. Braun, A. Lenz, N. Mahnke and E. Stein, Phys. Rev. D 65, 074011 (2002).

[36] I. I. Balitsky, V. M. Braun and A. V. Kolesnichenko, Nucl. Phys. B 312, 509 (1989).

[37] V. L. Chernyak and I. R. Zhitnitsky, Nucl. Phys. B 345, 137 (1990).

[38] V. M. Braun, A. Khodjamirian and M. Maul, Phys. Rev. D 61, 073004 (2000).

[39] J. Bijnens and A. Khodjamirian, Eur. Phys. J. C 26, 67 (2002).

[40] V. M. Braun, arXiv:hep-ph/9801222

[41] P. Colangelo and A. Khodjamirian, arXiv:hep-ph/0010175

[42] Z. G. Wang, S. L. Wan and W. M. Yang, arXiv:hep-ph/0601025

[43] Z. G. Wang, S. L. Wan and W. M. Yang, arXiv:hep-ph/0601060

[44] A. Lenz, M. Wittmann and E. Stein, Phys. Lett. B 581, 199 (2004).

[45] M. q. Huang and D. W. Wang, Phys. Rev. D 69, 094003 (2004).

[46] V. M. Braun, A. Lenz, G. Peters and A.V. Radyushkin, Phys. Rev. D 73 (2006) 034020.

[47] S. Eidelman et al. [Particle Data Group Collaboration], Phys. Lett. B 592 (2004) 1.

[48] R. C. Walker et al., Phys. Rev. D 49 (1994) 5671.

[49] L. Andivahis et al., Phys. Rev. D 50 (1994) 5491.

[50] J. Litt et al., Phys. Lett. B 31 (1970) 40.

[51] C. Berger, V. Burkert, G. Knop, B. Langenbeck and K. Rith, Phys. Lett. B 35 (1971) 87.

[52] T. Janssens, R. Hofstadter, E. B. Huges and 
M. R. Yearian, Phys. Rev. 142, 922 (1966).

[53] J. Arrington, Phys. Rev. C 68 (2003) 034325.

[54] M. E. Christy et al. [E94110 Collaboration], Phys. Rev. C 70 (2004) 015206.

[55] I. A. Qattan et al., Phys. Rev. Lett. 94 (2005) 142301.

[56] A. Lung et al., Phys. Rev. Lett. 70 (1993) 718.

[57] G. Kubon et al., Phys. Lett. B 524 (2002) 26.

[58] H. Anklin et al., Phys. Lett. B 428 (1998) 248.

[59] H. Zhou et al., Phys. Rev. Lett. 87 (2001), 0818011.

[60] D. Rohe, Phys. Rev. Lett. 83 (1999), 4257.

[61] L. C. Maximon and J. A. Tjon, Phys. Rev. C 62 (2000) 054320.

[62] P. G. Blunden, W. Melnitchouk and J. A. Tjon, Phys. Rev. Lett. 91 (2003) 142304.

[63] J. Arrington, Phys. Rev. C 69 (2004) 032201.

[64] Y. C. Chen, A. Afanasev, S. J. Brodsky, C. E. Carlson and M. Vanderhaeghen, Phys. Rev. Lett. 93 (2004) 122301.

[65] J. Arrington, Phys. Rev. C 71 (2005) 015202.

[66] E. Tomasi-Gustafsson and G. I. Gakh, Phys. Rev. C 72 (2005) 015209.

[67] A. V. Afanasev, S. J. Brodsky, C. E. Carlson, Y. C. Chen and M. Vanderhaeghen, Phys. Rev. D 72 (2005) 013008.

[68] S. Kondratyuk, P. G. Blunden, W. Melnitchouk and J. A. Tjon, Phys. Rev. Lett. 95 (2005) 172503.

[69] P. G. Blunden, W. Melnitchouk and J. A. Tjon, Phys. Rev. C 72 (2005) 034612.

[70] M. K. Jones et al. [Jefferson Lab Hall A Collaboration], Phys. Rev. Lett. 84 (2000) 1398;

V. Punjabi et al., Phys. Rev. C 71, 055202 (2005) [Erratum-ibid. C 71, 069902 (2005)].

[71] O. Gayou et al., Phys. Rev. C 64 (2001) 038202;

[72] O. Gayou et al. [Jefferson Lab Hall A Collaboration], Phys. Rev. Lett. 88 (2002) 092301.

[73] S. J. Barish et al., Phys. Rev. D 16 (1977) 3103.

[74] N. J. Baker et al., Phys. Rev. D 23 (1981) 2499.

[75] K. L. Miller et al., Phys. Rev. D 26 (1982) 537.
[76] T. Kitagaki et al., Phys. Rev. D 28 (1983) 436.

[77] H. Budd, A. Bodek and J. Arrington, arXiv:hep-ex/0308005

[78] A. Liesenfeld et al. [A1 Collaboration], Phys. Lett. B 468 (1999) 20.

[79] V. Bernard, L. Elouadrhiri and U. G. Meissner, J. Phys. G 28 (2002) R1.

[80] S. Choi et al., Phys. Rev. Lett. 71 (1993) 3927.

[81] L. A. Ahrens et al., Phys. Lett. B 202 (1988) 284.

[82] J. D. Bjorken and S. D. Drell, Relativistic Quantum Fields (McGraw-Hill, New York, 1965).

[83] V. Braun, R. J. Fries, N. Mahnke and E. Stein, Nucl. Phys. B 589 (2000) 381 [Erratum-ibid. B 607 (2001) 433].

[84] B. L. Ioffe, Z. Phys. C 18 (1983) 67.

[85] B. L. Ioffe, Nucl. Phys. B 188 (1981) 317 [Erratum-ibid. B 191 (1981) 591].

[86] H. G. Dosch, M. Jamin and S. Narison, Phys. Lett. B 220, 251 (1989).

[87] Y. Chung, H. G. Dosch, M. Kremer and D. Schall, Nucl. Phys. B 197 (1982) 55.

[88] V.L. Chernyak and I.R. Zhitnitsky, Nucl. Phys. B246 (1984) 52.

[89] A. R. Zhitnitsky, I. R. Zhitnitsky and V. L. Chernyak, Sov. J. Nucl. Phys. 41 (1985) 284 [Yad. Fiz. 41 (1985) 445].

[90] T. Huang and Z. H. Li, Phys. Rev. D 57 (1998) 1993.

[91] I.D. King and C.T. Sachrajda, Nucl. Phys. B279 (1987) 785.

[92] V.L. Chernyak, A.A. Ogloblin and I.R. Zhitnitsky, Sov. J. Nucl. Phys. 48 (1988) 536; Z. Phys. C 42 (1989) 583.

[93] J. Bolz and P. Kroll, Z. Phys. A 356 (1996) 327.

[94] I. I. Balitsky and V. M. Braun, Nucl. Phys. B 311, 541 (1989).

[95] I. I. Balitsky and V. M. Braun, Nucl. Phys. B 361 (1991) 93.

[96] P. Ball and V. M. Braun, Nucl. Phys. B 543 (1999) 201. 